\title{
Nickel and Platinum $\sigma$-Bonded Derivatives of Corannulene
}

\author{
Han Baek Lee and Paul R. Sharp*
}

125 Chemistry, University of Missouri, Columbia, MO 65211, USA

SharpP@missouri.edu

\section{Experimental}

Corannulene, ${ }^{1}$ bromocorannulene, ${ }^{1}$ tetrabromocorannulene, ${ }^{2}$ and $\mathrm{Pt}\left(\mathrm{PEt}_{3}\right)_{4}{ }^{3}$ were prepared by literature procedures. $\mathrm{PEt}_{3}\left(10 \%\right.$ wt hexane solution) and $\mathrm{Ni}(\mathrm{COD})_{2}$ were purchased from commercial sources (STREM) and used as received. Experiments were performed under a dinitrogen atmosphere in a Vacuum Atmospheres Corporation drybox or on a Schlenk line with dried and degassed solvents stored under dinitrogen and over $4 \AA$ molecular sieves or sodium metal. NMR spectra were recorded on a Bruker AMX-250, -300 , or -500 spectrometers at ambient probe temperatures unless otherwise stated. Shifts are given in ppm with positive values downfield of TMS $\left({ }^{1} \mathrm{H}\right.$ and ${ }^{13} \mathrm{C}$ ), external $\mathrm{H}_{3} \mathrm{PO}_{4}\left({ }^{31} \mathrm{P}\right)$ or $\mathrm{K}_{2} \mathrm{PtCl}_{4}\left({ }^{195} \mathrm{Pt}\right) .{ }^{13} \mathrm{C}$ and ${ }^{31} \mathrm{P}$ NMR spectra were recorded in proton decoupled mode. Desert Analytics performed the microanalyses (inert atmosphere). The presence of solvents of crystallization in the analyzed samples was confirmed by NMR spectroscopy.

${ }^{1}$ Seiders, T. J.; Elliott, E. L.; Grube, G. H.; Siegel, J. S. J. Am. Chem. Soc. 1999, 121, 7804-7813.

${ }^{2}$ Sygula A.; Rabideau, P. W. J. Am. Chem. Soc. 2000, 122, 6263-6264.

${ }^{3}$ Yoshida, T.; Matsuda, T.; Otsuka, S. Inorg. Synth. 1990, 28, 122-123. 
$\mathbf{N i}(\mathrm{Br})\left(\mathrm{PEt}_{\mathbf{3}}\right)_{\mathbf{2}}\left(\mathrm{C}_{\mathbf{2 0}} \mathrm{H}_{\mathbf{9}}\right)$ (1). Triethylphosphine in hexane (10\% wt, $\mathrm{d}=0.66 \mathrm{~g} / \mathrm{mL}, 109$ $\mu \mathrm{L}, 0.0608 \mathrm{mmol}$ ) was added to a stirred suspension of bromocorannulene $(10.0 \mathrm{mg}$, $0.0304 \mathrm{mmol})$ in $5.0 \mathrm{~mL}$ of toluene. The resulting solution was then added to $\mathrm{Ni}(\mathrm{COD})_{2}$ ( $8.36 \mathrm{mg}, 0.0304 \mathrm{mmol}$ ) dissolved in $3.0 \mathrm{~mL}$ of toluene. The mixture was stirred for 8 hours at room temperature, filtered through a diatomaceous earth pad, and the volatiles removed in vacuo. The solid residue was washed with cold hexane $(2 \mathrm{~mL})$ and dried in vacuo to give $16.1 \mathrm{mg}$ (84.8\%) of yellow-brown solid 1. Yellow-brown crystals of 1 for the X-ray and elemental analyses were obtained by dissolving the solid in toluene (1.5 $\mathrm{mL}$ ), adding $1.5 \mathrm{~mL}$ of hexane, and holding the mixture at $-20^{\circ} \mathrm{C}$ overnight.

Anal. Calcd. (Found) for $\mathrm{C}_{32} \mathrm{H}_{39} \mathrm{BrP}{ }_{2} \mathrm{Ni}$ : C: 61.53 (61.92), H: 6.25 (6.32).

${ }^{31} \mathrm{P}\{\mathrm{H}\}$ NMR (101 MHz, $\left.\mathrm{C}_{6} \mathrm{D}_{6}\right): 11.22(\mathrm{~s}) .{ }^{31} \mathrm{P}\{\mathrm{H}\} \mathrm{NMR}\left(101 \mathrm{MHz}\right.$, toluene, $\left.-70{ }^{\circ} \mathrm{C}\right): 12.76$ (s, exo or endo $\mathrm{PEt}_{3}$ ), 11.70 (s, exo or endo $\left.\mathrm{PEt}_{3}\right), \mathrm{T}_{\mathrm{c}}=-23^{\circ} \mathrm{C}$.

${ }^{1} \mathrm{H}$ NMR $\left(300 \mathrm{MHz}, \mathrm{C}_{6} \mathrm{D}_{6}\right): 8.79\left(\mathrm{~d}, \mathrm{~J}_{\mathrm{HH}}=8.7 \mathrm{~Hz}, 1 \mathrm{H}\right), 7.88(\mathrm{~s}, 1 \mathrm{H}), 7.77\left(\mathrm{~d}, \mathrm{~J}_{\mathrm{HH}}=8.7 \mathrm{~Hz}\right.$, $1 \mathrm{H}), 7.61(\mathrm{~m}, 3 \mathrm{H}), 7.51\left(\mathrm{~d}, \mathrm{~J}_{\mathrm{HH}}=8.7 \mathrm{~Hz}, 1 \mathrm{H}\right), 7.49\left(\mathrm{~d}, \mathrm{~J}_{\mathrm{HH}}=8.7 \mathrm{~Hz}, 1 \mathrm{H}\right), 7.39\left(\mathrm{~d}, \mathrm{~J}_{\mathrm{HH}}=\right.$ $8,7 \mathrm{~Hz}, 1 \mathrm{H}), 1.35-1.11\left(\mathrm{~m}, 12 \mathrm{H}, \mathrm{C}_{2} \mathrm{CH}_{3}\right)$ 0.88-0.78 (m, 18H, $\left.\mathrm{CH}_{2} \underline{\mathrm{C}}_{3}\right)$.

${ }^{13} \mathrm{C}$ NMR $\left(76 \mathrm{MHz}, \mathrm{C}_{6} \mathrm{D}_{6}\right): 165.07(\mathrm{t}, \mathrm{JPC}=33.13 \mathrm{~Hz}) 137.92(\mathrm{t}, \mathrm{JPC}=2.34 \mathrm{~Hz}) 136.97$ (s) 136.16 (s) 135.73 (s) 134.56 (s) 133.53 (s) 131.43 (s) 131.37 (s) 131.06 (t, JPC = $2.94 \mathrm{~Hz}) 130.53$ (s) 130.07 (t, JPC = 5.28 Hz) 129.77 (s) 127.39 (s) 127.28 (s) 127.26 (s) 126.91 (s) 126.36 (s) 125.66 (s) 125.51 (s) 15.03 (t, JPC $\left.=12.81 \mathrm{~Hz}, \underline{\mathrm{CH}_{2}} \mathrm{CH}_{3}\right), 8.25$ (s, $\mathrm{CH}_{2} \underline{\mathrm{C}} \mathrm{H}_{3}$ ).

$\mathbf{P t}(\mathrm{Br})\left(\mathrm{PEt}_{\mathbf{3}}\right)_{\mathbf{2}}\left(\mathrm{C}_{\mathbf{2 0}} \mathrm{H}_{\mathbf{9}}\right)$ (2). Tetrakis(triethylphosphine)platinum(0) (20.3 mg, 0.0304 $\mathrm{mmol})$ in $5.0 \mathrm{~mL}$ of toluene was added to bromocorannulene $(10.0 \mathrm{mg}, 0.0304 \mathrm{mmol})$ in $5.0 \mathrm{~mL}$ of toluene. The resulting solution was stirred for $12 \mathrm{hrs}$ at room temperature, filtered through a diatomaceous earth pad, and the volatiles removed in vacuo. The solid residue was washed with cold hexane $(2 \mathrm{~mL})$ and dried in vacuo to give $18.3 \mathrm{mg}$ 
(79.2 \%) of yellow solid 2. Yellow crystals of $\mathbf{2}$ for the X-ray were obtained by dissolving the solid in toluene $(1.5 \mathrm{~mL})$, adding $1.5 \mathrm{~mL}$ of hexane, and holding the mixture at room temperature overnight.

${ }^{31} \mathrm{P}\{\mathrm{H}\}$ NMR (101 MHz, toluene): 12.63 (s with satellites, $\left.\mathrm{J}_{\mathrm{PtP}}=2699 \mathrm{~Hz}\right) .{ }^{31} \mathrm{P}\{\mathrm{H}\} \mathrm{NMR}$ $\left(101 \mathrm{MHz}\right.$, toluene, $-70^{\circ} \mathrm{C}$ ): 13.67 (s with satellites, $\mathrm{J}_{\mathrm{PtP}}=2672 \mathrm{~Hz}$, exo or endo $\mathrm{PEt}_{3}$ ), 13.21 ( $\mathrm{s}$ with satellites, $\mathrm{J}_{\mathrm{PtP}}=2666 \mathrm{~Hz}$, exo or endo $\mathrm{PEt}_{3}$ ), $\mathrm{T}_{\mathrm{c}}=-28^{\circ} \mathrm{C}$.

${ }^{1} \mathrm{H}$ NMR (300 MHz, $\left.\mathrm{C}_{6} \mathrm{D}_{6}\right): 8.41\left(\mathrm{~d}, \mathrm{~J}_{\mathrm{HH}}=8.7 \mathrm{~Hz}, 1 \mathrm{H}\right), 8.09\left(\mathrm{~s}, \mathrm{~J}_{\mathrm{PtH}}=\mathrm{Hz}, 1 \mathrm{H}\right), 7.78(\mathrm{~d}$, $\left.J_{H H}=8.7 \mathrm{~Hz}, 1 \mathrm{H}\right), 7.66(\mathrm{~m}, 3 \mathrm{H}), 7.50\left(\mathrm{~d}, \mathrm{~J}_{\mathrm{HH}}=8.7 \mathrm{~Hz}, 1 \mathrm{H}\right), 7.49\left(\mathrm{~d}, \mathrm{~J}_{\mathrm{HH}}=8.7 \mathrm{~Hz}, 1 \mathrm{H}\right)$, $7.41\left(\mathrm{~d}, \mathrm{~J}_{\mathrm{HH}}=8.7 \mathrm{~Hz}, 1 \mathrm{H}\right), 1.58-1.35\left(\mathrm{~m}, 12 \mathrm{H}, \mathrm{C}_{2} \mathrm{CH}_{3}\right) 0.82-0.72\left(\mathrm{~m}, 18 \mathrm{H}, \mathrm{CH}_{2} \mathrm{C}_{3}\right)$.

${ }^{13} \mathrm{C}$ NMR (76 MHz, $\mathrm{C}_{6} \mathrm{D}_{6}$ ): 147.10 (t, JPC $\left.=9.17 \mathrm{~Hz}\right) 138.25$ (s) 136.91 (s) 136.10 (s) 136.08 (s) 135.30 (s) 133.40 (s) 133.19 (s) 132.11 (s) 131.57 (s) 130.72 (s) 130.39 (t, $\left.\mathrm{J}_{\mathrm{PC}}=2.57 \mathrm{~Hz}\right) 129.94$ (s) 127.36 (s) 127.32 (s) 127.29 (s) 126.90 (s) 126.34 (s) 126.13 (s) $125.24(\mathrm{~s}) 14.52$ (q, JPC $\left.=16.88 \mathrm{~Hz}, \underline{\mathrm{CH}_{2}} \mathrm{CH}_{3}\right), 7.79$ (t, $\mathrm{J}_{\mathrm{PC}}=25.72 \mathrm{~Hz}, \mathrm{CH}_{2} \underline{\mathrm{C}} \mathrm{H}_{3}$ ).

Anal. Calcd. for $\mathrm{C}_{32} \mathrm{H}_{39} \mathrm{BrP}{ }_{2} \mathrm{Pt}$ : C, 50.53; $\mathrm{H}, 5.17$. Highly variable low values were found: C, 47.14, 48.34, 42.82; H, 4.96, 5.06, 5.38.

cis- $\mathrm{Pt}(\mathrm{Br})\left(\mathrm{PEt}_{3}\right)_{\mathbf{2}}\left(\mathrm{C}_{20} \mathrm{H}_{6} \mathrm{Br}_{3}\right)$ (cis-3, cis-4). Tetrabromocorannulene (20.0 mg, 0.0353 $\mathrm{mmol}$ ) was dissolved completely in $80 \mathrm{~mL}$ of toluene. (Complex mixtures are obtained if the tetrabromocorannulene is not completely dissolved.)

Tetrakis(triethylphosphine)platinum(0) $(23.8 \mathrm{mg}, 0.0353 \mathrm{mmol})$ in $3.0 \mathrm{~mL}$ of toluene was slowly added drop wise to the stirred solution. The mixture was stirred for $24 \mathrm{hrs}$ at room temperature, concentrated to $10 \mathrm{~mL}$ under the reduced pressure, filtered through a diatomaceous earth pad, and the volatiles removed in vacuo. The residue was washed with cold hexane $(2.0 \mathrm{~mL})$ and dried in vacuo to give a yellow solid $(18.0 \mathrm{mg}$, $51.1 \%)$ consisting of a mixture of cis-3 and cis-4. 
${ }^{31} \mathrm{P}\{\mathrm{H}\}$ NMR $\left(101 \mathrm{MHz}, \mathrm{CD}_{2} \mathrm{Cl}_{2}\right)$ : Isomer A: 5.80 (d with satellite, $\mathrm{JPtP}_{\mathrm{Pt}}=1861 \mathrm{~Hz}, \mathrm{~J}_{\mathrm{PP}}=$ 18.2 Hz), 1.69 (d with satellite, $J_{P t P}=3989 \mathrm{~Hz}, J_{P P}=17.9 \mathrm{~Hz}$ ). Isomer B: 5.75 (d with satellite, $J_{P t P}=1857 \mathrm{~Hz}, J_{P P}=18.2 \mathrm{~Hz}$ ), 1.57 (d with satellite, $J_{P t P}=3993 \mathrm{~Hz}, J_{P P}=18.2$ $\mathrm{Hz}$ ). Isomer identity has not been assigned.

trans- $\mathrm{Pt}(\mathrm{Br})\left(\mathrm{PEt}_{3}\right)_{2}\left(\mathrm{C}_{20} \mathrm{H}_{6} \mathrm{Br}_{3}\right)$ (trans-3, trans-4). Tetrabromocorannulene (20.0 mg, $0.0353 \mathrm{mmol}$ ) was dissolved completely in $80.0 \mathrm{~mL}$ of toluene. (Complex mixtures are obtained if the tetrabromocorannulene is not completely dissolved.) Tetrakis(triethylphosphine)platinum(0) $(23.8 \mathrm{mg}, 0.0353 \mathrm{mmol}$ ) in $5.0 \mathrm{~mL}$ of toluene was slowly added drop wise to the stirred solution. The mixture was sealed in a reaction vessel and stirred for $24 \mathrm{hrs}$ at room temperature and then heated to $130^{\circ} \mathrm{C}$ for $24 \mathrm{hrs}$. The resulting solution was concentrated to $10 \mathrm{~mL}$ under the reduced pressure, filtered through a diatomaceous earth pad, and the volatiles removed in vacuo. The residue was washed with cold hexane $(2.0 \mathrm{~mL})$ and dried in vacuo to give a yellow solid (24.6 $\mathrm{mg}, 69.9 \%$ ) consisting of a mixture of trans-3 and trans-4. Yellow crystals for the first Xray analysis, consisting of a 1:2 mixture of trans-3 to trans-4, were obtained by slow evaporation of a concentrated toluene solution $(3.0 \mathrm{ml})$ of the isolated mixture. The isomeric mixture of trans-3, trans-4 was separated by chromatography (silica, $50 \%$ toluene $/ 50 \%$ cyclohexane) with trans-3 first to elute.

${ }^{31} \mathrm{P}\{\mathrm{H}\}$ NMR $\left(101 \mathrm{MHz}, \mathrm{C}_{6} \mathrm{D}_{6}\right)$ : trans-3: 9.47 (s with satellites, $\mathrm{J}_{\mathrm{PtP}}=2624 \mathrm{~Hz}$ ), trans-4: 9.32 (s with satellites, $\mathrm{JPtP}_{\mathrm{P}}=2628 \mathrm{~Hz}$ ).

${ }^{1} \mathrm{H}$ NMR (500 MHz, $\left.\mathrm{C}_{6} \mathrm{D}_{6}\right)$ : trans-3: $8.44\left(\mathrm{~d}, \mathrm{~J}_{\mathrm{HH}}=8.8 \mathrm{~Hz}, 1 \mathrm{H}\right), 8.04\left(\mathrm{dd}, \mathrm{J}_{\mathrm{HH}}=8.8 \mathrm{~Hz}\right.$, $2 \mathrm{H}), 7.51\left(\mathrm{~d}, \mathrm{~J}_{\mathrm{HH}}=8.8 \mathrm{~Hz}, 1 \mathrm{H}\right), 7.47\left(\mathrm{~d}, \mathrm{~J}_{\mathrm{HH}}=8.8 \mathrm{~Hz}, 1 \mathrm{H}\right), 7.25\left(\mathrm{~d}, \mathrm{~J}_{\mathrm{HH}}=8.8 \mathrm{~Hz}, 1 \mathrm{H}\right)$, 1.66-1.42 (m, $\left.12 \mathrm{H}, \underline{\mathrm{CH}}_{2} \mathrm{CH}_{3}\right), 0.84-0.77\left(\mathrm{~m}, 18 \mathrm{H}, \mathrm{CH}_{2} \underline{\mathrm{C}}_{3}\right)$; trans-4: $8.43\left(\mathrm{~d}, \mathrm{~J}_{\mathrm{HH}}=8.8\right.$ $\mathrm{Hz}, 1 \mathrm{H}), 8,01\left(\mathrm{~d}, \mathrm{~J}_{\mathrm{HH}}=8.8 \mathrm{~Hz}, 1 \mathrm{H}\right), 7.84\left(\mathrm{~d}, \mathrm{~J}_{\mathrm{HH}}=8.8 \mathrm{~Hz}, 1 \mathrm{H}\right), 7.64\left(\mathrm{~d}, \mathrm{~J}_{\mathrm{HH}}=8.8 \mathrm{~Hz}\right.$, $1 \mathrm{H}), 7.58\left(\mathrm{~d}, \mathrm{~J}_{\mathrm{HH}}=8.8 \mathrm{~Hz}, 1 \mathrm{H}\right), 7.42\left(\mathrm{~d}, \mathrm{~J}_{\mathrm{HH}}=8.8 \mathrm{~Hz}, 1 \mathrm{H}\right), 1.70-1.44\left(\mathrm{~m}, 12 \mathrm{H}, \mathrm{C}_{2} \mathrm{CH}_{3}\right)$, 0.88-0.76 (m, 18H, $\left.\mathrm{CH}_{2} \underline{\mathrm{C}}_{3}\right)$. 
Anal. Calcd (Found) for the isomeric mixture $\mathrm{C}_{32} \mathrm{H}_{36} \mathrm{Br}_{4} \mathrm{P}_{2} \mathrm{Pt}$ : C, 38.54 (38.68); $\mathrm{H}, 3.64$ (3.53).

trans-[Pt(PEt $\left.)_{2}(\mathrm{Br})\right]_{2}\left(\mathrm{C}_{20} \mathrm{H}_{6} \mathrm{Br}_{2}\right)$ (trans-5, trans-6, trans-7). Tetrabromocorannulene (20.0 mg, $0.0353 \mathrm{mmol}$ ) was completely dissolved in $80.0 \mathrm{~mL}$ of toluene. (Complex mixtures are obtained if the tetrabromocorannulene is not completely dissolved.) Tetrakis(triethylphosphine)platinum(0) $(47.1 \mathrm{mg}, 0.0706 \mathrm{mmol})$ in $3.0 \mathrm{~mL}$ of toluene was slowly added drop wise to the stirred solution. The mixture was sealed in a reaction vessel and stirred for $24 \mathrm{hrs}$ at room temperature and then heated to $130^{\circ} \mathrm{C}$ for $24 \mathrm{hrs}$. The resulting solution was concentrated to $10 \mathrm{~mL}$ under the reduced pressure, filtered through a diatomaceous earth pad, and the volatiles removed in vacuo. The residue was washed with cold hexane $(2.0 \mathrm{ml})$ and dried in vacuo to give a yellow solid (41.0

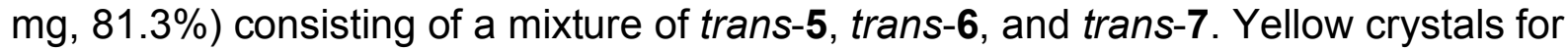
the X-ray analysis were obtained by slow evaporation or hexane layering of a concentrated toluene solution of the mixture. The isomeric mixture was separated by chromatography (silica, $25 \%$ toluene/75\% cyclohexane) with trans- 6 first to elute followed by trans-5, and then trans-7. Small amounts of trans-3 and trans-4 eluted much earlier.

${ }^{31} \mathrm{P}\{\mathrm{H}\}$ NMR (101 MHz, $\mathrm{C}_{6} \mathrm{D}_{6}$ ) of trans-5, trans-6, and trans-7 mixture: 9.33 (s with satellites, $\mathrm{J}_{\mathrm{PtP}} \sim 2660 \mathrm{~Hz}$ ), 9.20 (s with satellites, $\mathrm{J}_{\mathrm{PtP}} \sim 2660 \mathrm{~Hz}$ ), 9.18 (s with satellites, $J_{\text {PtP }} \sim 2660 \mathrm{~Hz}$ ). Traces of trans-8 can be observed in the mixture at 12.8 along with three other peaks in the same region attributed to the 3 other possible singly debrominated isomers.

${ }^{31} \mathrm{P}\{\mathrm{H}\}$ NMR (101 MHz, toluene) of crystals: 9.14 (s with satellites, $\mathrm{J}_{\mathrm{PtP}}=2664 \mathrm{~Hz}$, trans5); 12.83 ( $\mathrm{s}$ with satellites, $\mathrm{J}_{\mathrm{PtP}}=2706 \mathrm{~Hz}$, trans-8). The second peak for trans-8 is assumed to overlap with that of trans-5 at 9.14 . With this assumption the integration ratio of trans-5 to trans-8 is $3: 2$. 
${ }^{31} \mathrm{P}\{\mathrm{H}\}$ NMR (101 MHz, $\mathrm{C}_{6} \mathrm{D}_{6}$ ) of separated isomers: trans-5, 9.27 (s with satellites, J JPtP $=2657 \mathrm{~Hz}$ ); trans-6, 9.38 \& 9.34 (overlapping singlets with satellites, $\mathrm{J}_{\mathrm{PtP}}=2657 \mathrm{~Hz}$ ); trans-7, 9.29 (s with satellites, J $\mathrm{PtP}_{\mathrm{P}}=2657 \mathrm{~Hz}$ ).

${ }^{1} \mathrm{H}$ NMR $\left(500 \mathrm{MHz}, \mathrm{C}_{6} \mathrm{D}_{6}\right)$ of crystals: trans-5; $8.51(\mathrm{~s}, 2 \mathrm{H}), 8.03\left(\mathrm{~d}, \mathrm{~J}_{\mathrm{HH}}=8.8 \mathrm{~Hz}, 2 \mathrm{H}\right)$, $7.51\left(\mathrm{~d}, \mathrm{~J}_{\mathrm{HH}}=8.8 \mathrm{~Hz}, 2 \mathrm{H}\right), 1.71-1.53\left(\mathrm{~m}, 12 \mathrm{H}, \mathrm{C}_{2} \mathrm{CH}_{3}\right), 0.88-0.72\left(\mathrm{~m}, 18 \mathrm{H}, \mathrm{CH}_{2} \underline{\mathrm{C}}_{3}\right)$, trans-8; $8.52\left(\mathrm{~d}, \mathrm{~J}_{\mathrm{HH}}=8.7 \mathrm{~Hz}, 2 \mathrm{H}\right), 8.44\left(\mathrm{~d}, \mathrm{~J}_{\mathrm{HH}}=8.7 \mathrm{~Hz}, 2 \mathrm{H}\right), 8.07\left(\mathrm{~d}, \mathrm{~J}_{\mathrm{HH}}=8.7 \mathrm{~Hz}, 2 \mathrm{H}\right)$ $8.06(\mathrm{~s}, 1 \mathrm{H}), 7.59\left(\mathrm{~d}, \mathrm{~J}_{\mathrm{HH}}=8.5 \mathrm{~Hz}, 2 \mathrm{H}\right), 7.58\left(\mathrm{~d}, \mathrm{~J}_{\mathrm{HH}}=8.7 \mathrm{~Hz}, 2 \mathrm{H}\right), 7.53\left(\mathrm{~d}, \mathrm{~J}_{\mathrm{HH}}=8.6 \mathrm{~Hz}\right.$, $2 \mathrm{H})$. The $\mathrm{PEt}_{3}$ peaks for trans-8 are assumed to overlap with those for trans-5.

${ }^{1} \mathrm{H}$ NMR $\left(500 \mathrm{MHz}, \mathrm{C}_{6} \mathrm{D}_{6}\right)$ of separated isomers: trans-5: $8.50(\mathrm{~s}, 2 \mathrm{H}), 8.03\left(\mathrm{~d}, \mathrm{~J}_{\mathrm{HH}}=\right.$ $8.50 \mathrm{~Hz}, 2 \mathrm{H}), 7.51\left(\mathrm{~d}, \mathrm{~J}_{\mathrm{HH}}=8.50 \mathrm{~Hz}, 2 \mathrm{H}\right), 1.66-1.54\left(\mathrm{~m}, 24 \mathrm{H}, \mathrm{C}_{2} \mathrm{CH}_{3}\right), 0.86-0.72(\mathrm{~m}$, $\left.36 \mathrm{H}, \mathrm{CH}_{2} \mathrm{C}_{3}\right)$, trans-6: $8.42\left(\mathrm{~d}, \mathrm{~J}_{\mathrm{HH}}=8.50 \mathrm{~Hz}, 1 \mathrm{H}\right), 8.41\left(\mathrm{~d}, \mathrm{~J}_{\mathrm{HH}}=8.50 \mathrm{~Hz}, 1 \mathrm{H}\right), 8.08(\mathrm{~d}$, $\left.\mathrm{J}_{\mathrm{HH}}=8.50 \mathrm{~Hz}, 1 \mathrm{H}\right), 8.02\left(\mathrm{~d}, \mathrm{~J}_{\mathrm{HH}}=8.50 \mathrm{~Hz}, 1 \mathrm{H}\right), 7.62\left(\mathrm{~d}, \mathrm{~J}_{\mathrm{HH}}=8.50 \mathrm{~Hz}, 1 \mathrm{H}\right), 7.54\left(\mathrm{~d}, \mathrm{~J}_{\mathrm{HH}}\right.$ $=9.00 \mathrm{~Hz}, 1 \mathrm{H}), 1.65-1.52\left(\mathrm{~m}, 24 \mathrm{H}, \mathrm{C}_{2} \mathrm{CH}_{3}\right), 0.83-0.74\left(\mathrm{~m}, 36 \mathrm{H}, \mathrm{CH}_{2} \underline{\mathrm{H}}_{3}\right)$, trans-7: 8.42 $\left(\mathrm{d}, \mathrm{J}_{\mathrm{HH}}=8.50 \mathrm{~Hz}, 2 \mathrm{H}\right), 8.03(\mathrm{~s}, 2 \mathrm{H}), 7.62\left(\mathrm{~d}, \mathrm{~J}_{\mathrm{HH}}=8.50 \mathrm{~Hz}, 2 \mathrm{H}\right), 1.64-1.53(\mathrm{~m}, 24 \mathrm{H}$, $\left.\mathrm{C}_{2} \mathrm{CH}_{3}\right), 0.82-0.72\left(\mathrm{~m}, 36 \mathrm{H}, \mathrm{CH}_{2} \underline{\mathrm{CH}}_{3}\right)$.

Anal. Calcd (Found) for the isomeric mixture $\mathrm{C}_{44} \mathrm{H}_{66} \mathrm{Br}_{4} \mathrm{P}_{4} \mathrm{Pt}_{2} \cdot \mathrm{C}_{7} \mathrm{H}_{8}: \mathrm{C}, 40.28$ (40.09); $\mathrm{H}$, 4.90 (4.75).

X-ray Analyses (all structures). Crystals were mounted by transferring the crystals from the mother liquor into a pool of heavy oil. A suitable crystal was selected and removed from the oil with a glass fiber. With the oil covered crystal adhering to the end of the glass fiber the sample was transferred to an $\mathrm{N}_{2}$ cold stream on the diffractometer and data were collected at $-100{ }^{\circ} \mathrm{C}$. Data reduction and processing followed routine procedures except as noted below. Structures were solved by direct methods.

Siemens SMART CCD: The data collection nominally covered over a hemisphere of reciprocal space, by a combination of three sets of exposures; each set had a different 
If angle for the crystal and each exposure covered $0.3^{\circ}$ in $\omega$. The crystal-to-detector distance was $5.00 \mathrm{~cm}$. Coverage of the unique set is over $95 \%$ complete to at least $26^{\circ}$ in $\theta$. Crystal decay was monitored by repeating the initial frames at the end of data collection and analyzing the duplicate reflections. No decay was detected. Data were corrected for absorption using the program SADABS based on the method of R.H. Blessing (Acta Cryst. A51, 33-38, 1995). Structures were solved by indirect methods. Refinement of $F^{2}$ against all reflections. The weighted $R$-factor $W R$ and goodness of fit $S$ are based on $\mathrm{F}^{2}$, conventional $\mathrm{R}$-factors $\mathrm{R}$ are based on $\mathrm{F}$, with $\mathrm{F}$ set to zero for negative $F^{2}$. The threshold expression of $F^{2}>2 \operatorname{sigma}\left(F^{2}\right)$ is used only for calculating $R$ factors (gt) etc. and is not relevant to the choice of reflections for refinement. R-factors based on $\mathrm{F}^{2}$ are statistically about twice as large as those based on $\mathrm{F}$, and $\mathrm{R}$ - factors based on ALL data will be even larger.

Complexes 1 and 2: Structure solution and refinement proceeded smoothly with no problems encountered. A drawing of 1 is shown in Fig S1. 


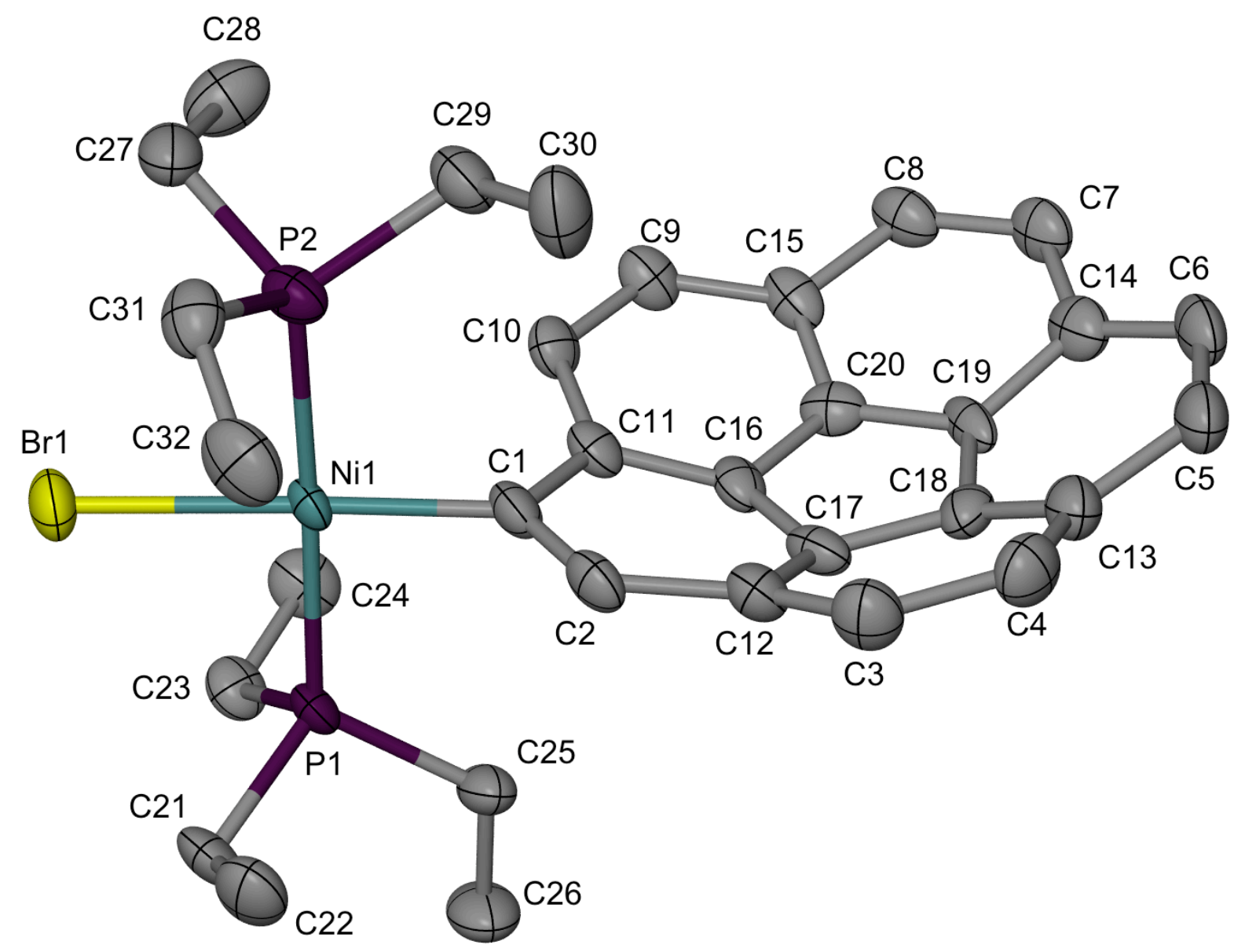

Figure S1. Drawing of complex 1 (50\% thermal ellipsoids, hydrogen atoms omitted for clarity).

Complex trans-3/trans-4: The $b$ and c axes for this crystal at $>30 \AA$ are at the resolution limit for the instrument. Some reflection overlap is likely and may explain the poor averaging statistics $(R($ int $)=30 \%)$. Nevertheless, the structure was readily solved. $\mathrm{Pt}, \mathrm{P}$, and the Pt-bound $\mathrm{Br}$ positions for 4 independent sites were apparent in the initial solution and early in the structure refinement. A complex web of peaks was apparent in the region expected for the corannulene fragment. In addition, five large peaks instead of the expected three were observed for the carbon-bound bromine atoms. This was interpreted as a disorder in the corannulene portions. To solve the disorder problem a corannulene fragment (carbon only) was generated in Chem3D and fitted to the major carbon peaks using the FRAG command to generate a rigid group in SHELXL. 
Subsequent difference Fouriers revealed a partial second corannulene fragment for each independent site. The second corannulenes were also fitted with the ridged group and their occupancy tied to the first corannulenes through free variables (total occupancy of 1). The carbon-bound bromine atoms were matched to the corannulene fragments and their occupancies also tied to the free variables. Examination of the refined positions of the corannulene fragments suggested that a second position for the Pt-bound bromine atom and one of the corannulene bound bromine atoms very close to the first position should be present. In 3 of the four independent molecules it was possible to identify these second positions in difference Fouriers and the second bromine positions were included in subsequent refinement with both positions again linked to the occupancy free variables. SIMU and SADI restraints were added as needed to keep the model stable to refinement. The corannulene carbon atoms, a couple of the lowest occupancy bromine atoms, and a few of the phosphine ethyl group carbon atoms were refined isotropically. Hydrogen atoms were added to complete the structures although they had little effect on the R-factor. The final isomer occupancies for the four independent sites are listed in Table S1. A drawing showing the disorder at site 1 is shown in Figure S2.

Table S1. Occupancy data for trans-3/trans-4

\begin{tabular}{|l|l|l|l|l|}
\hline & Site 1 & Site 2 & Site 3 & Site 4 \\
\hline trans-3 & 0.60 & 0.30 & 0.21 & 0.14 \\
\hline trans-4 & 0.40 & 0.70 & 0.79 & 0.86 \\
\hline
\end{tabular}




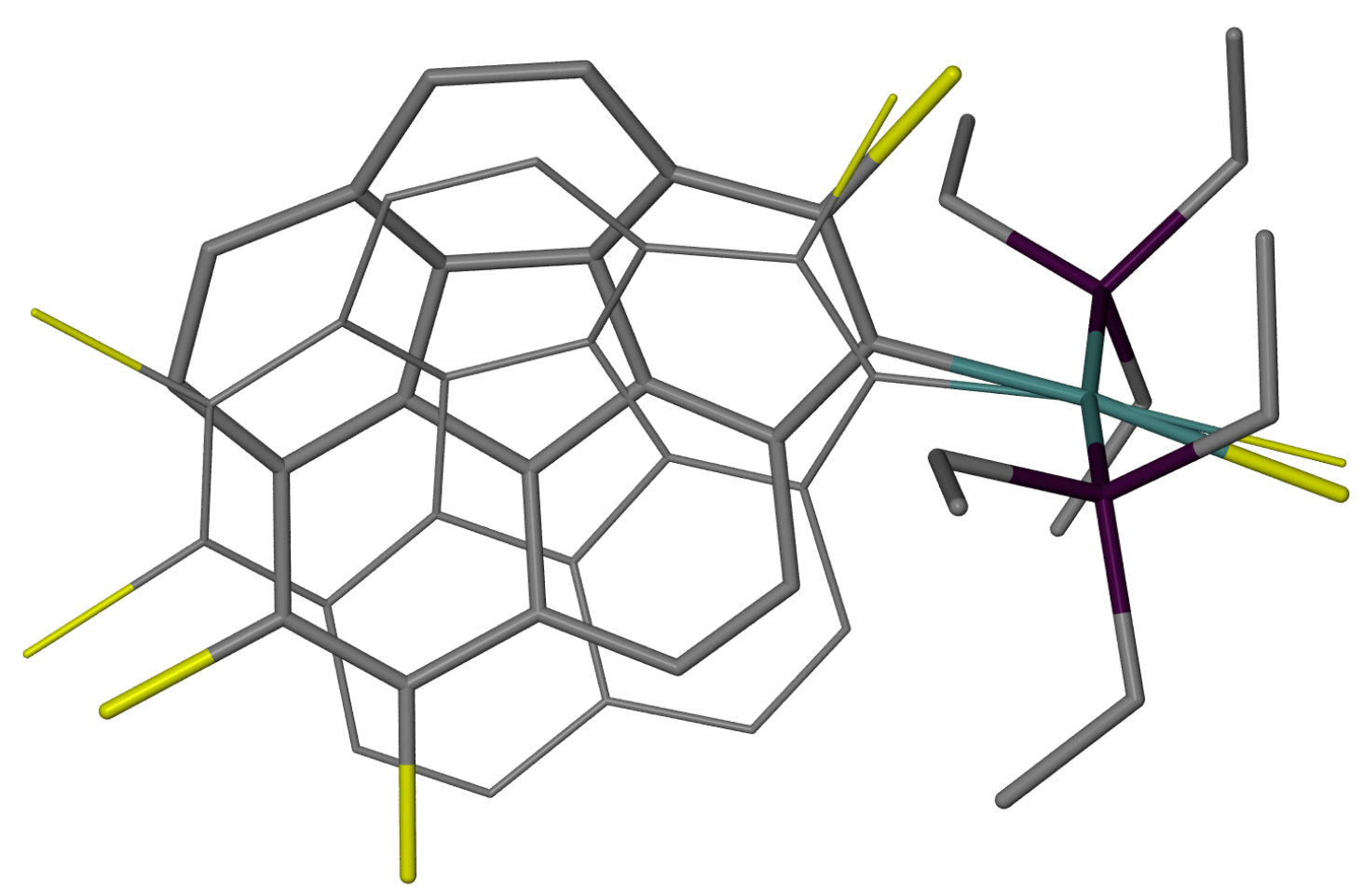

Figure S2. Drawing of the disorder at site 1. Thicker lines indicate greater occupancy.

Complex trans-5. During refinement of the structure an unreasonably large thermal parameter was evident for $\mathrm{Br} 1$ suggesting only partial occupancy. Occupancy refinement resulted in a significantly lower $R$ factor and better behaved thermal parameters for all of the atoms (no non-positive definite). Examination of the NMR data of the crystals indicated the presence of a debrominated form where the bromine atom corresponding to $\mathrm{Br} 1$ had been replaced by a hydrogen atom. Inclusion of the hydrogen atom (H1) with its occupancy linked to that of Br1 gave a final Br1 occupancy of $63.1 \%$ with a corresponding $\mathrm{H} 1$ occupancy of $36.9 \%$. This matched very well with the NMR ratio of 60:44. Disorder in the P2 and P3 phosphine ethyl groups was also evident and was modeled with two orientations with linked refined occupancy factors (major orientation for $\mathrm{P} 2$ at $68 \%$ and $50 \%$ for $\mathrm{P} 3$ ). Some of the carbon atom positions were shared between the two orientations. A few of the ethyl group carbon atoms went nonpositive definite on anisotropic refinement and were refined isotropically. 
Table 1. Crystal data and structure refinement for L2NiBr(corannulenyl)_1.

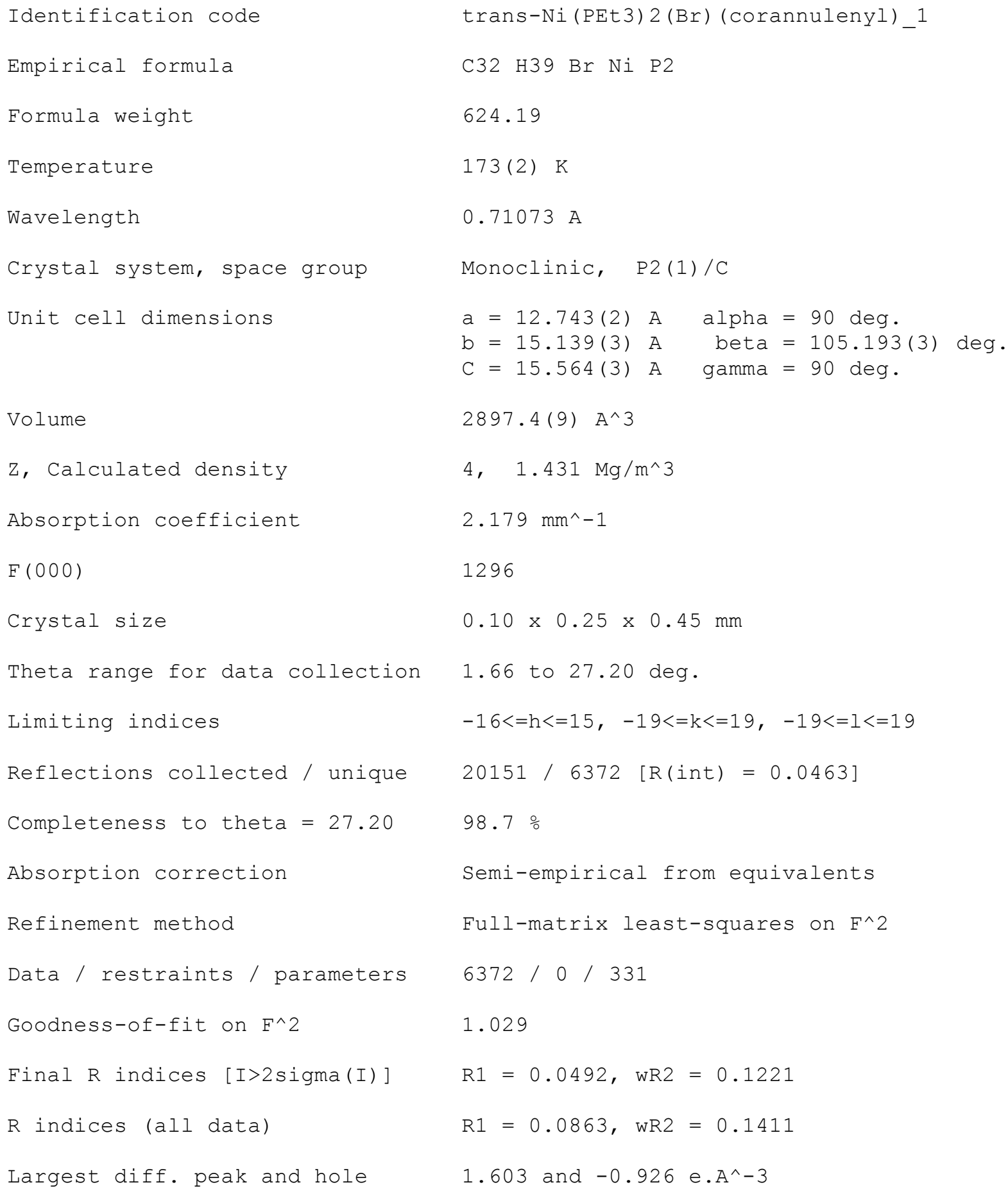


Table 2. Atomic coordinates $\left(x 1^{\wedge} 4\right)$ and equivalent isotropic displacement parameters $\left(A^{\wedge} 2 \times 10^{\wedge} 3\right)$ for L2NiBr(corannulenyl) 1. $\mathrm{U}(e q)$ is defined as one third of the trace of the orthogonalized Uij tensor.

\begin{tabular}{|c|c|c|c|c|}
\hline & $x$ & $\mathrm{y}$ & z & $\mathrm{U}(\mathrm{eq})$ \\
\hline $\operatorname{Br}(1)$ & $5487(1)$ & $6822(1)$ & $929(1)$ & $45(1)$ \\
\hline $\mathrm{Ni}(1)$ & $7284(1)$ & $6594(1)$ & $1787(1)$ & $26(1)$ \\
\hline$P(1)$ & $7025(1)$ & $5149(1)$ & $1591(1)$ & $30(1)$ \\
\hline$C(1)$ & $8716(3)$ & $6367(3)$ & $2454(3)$ & $36(1)$ \\
\hline$P(2)$ & $7456(1)$ & $8037(1)$ & $2018(1)$ & $36(1)$ \\
\hline$C(11)$ & $9580(3)$ & $6286(3)$ & $2014(3)$ & $34(1)$ \\
\hline$C(10)$ & $9649(4)$ & $6619(3)$ & $1175(3)$ & $37(1)$ \\
\hline C (9) & $10639(4)$ & $6702(3)$ & $951(3)$ & $41(1)$ \\
\hline$C(15)$ & $11658(4)$ & $6465(3)$ & $1553(3)$ & $39(1)$ \\
\hline$C(8)$ & $12752(4)$ & $6725(3)$ & $1601(4)$ & $44(1)$ \\
\hline$C(7)$ & $13590(4)$ & $6676(3)$ & $2402(3)$ & $45(1)$ \\
\hline$C(14)$ & $13402(4)$ & $6365(3)$ & $3205(3)$ & $44(1)$ \\
\hline C (6) & $13992(4)$ & $6530(3)$ & $4099(4)$ & $48(1)$ \\
\hline C (5) & $13498(4)$ & $6410(3)$ & $4810(4)$ & $49(1)$ \\
\hline$C(13)$ & $12392(4)$ & $6125(3)$ & $4672(3)$ & $43(1)$ \\
\hline C (4) & $11668(4)$ & $6230(3)$ & $5214(3)$ & $46(1)$ \\
\hline C (3) & $10514(4)$ & $6178(3)$ & $4852(3)$ & $46(1)$ \\
\hline$C(12)$ & $10037(3)$ & $6044(3)$ & $3935(3)$ & $39(1)$ \\
\hline C (2) & $8962(3)$ & $6243(3)$ & $3396(3)$ & $36(1)$ \\
\hline$C(16)$ & $10552(3)$ & $5941(2)$ & $2535(3)$ & $34(1)$ \\
\hline$C(20)$ & $11547(4)$ & $6036(3)$ & $2315(3)$ & $37(1)$ \\
\hline C (19) & $12381(3)$ & $5983(2)$ & $3094(3)$ & $38(1)$ \\
\hline$C(18)$ & $11910(3)$ & $5866(3)$ & $3811(3)$ & $36(1)$ \\
\hline C (17) & 10777 (3) & $5827(3)$ & $3446(3)$ & $36(1)$ \\
\hline C (21) & $5895(4)$ & $4756(3)$ & $2018(3)$ & $46(1)$ \\
\hline$C(22)$ & $6008(4)$ & $5023(3)$ & $2968(3)$ & $54(1)$ \\
\hline C (23) & $6635(4)$ & $4837(3)$ & $429(3)$ & $43(1)$ \\
\hline C (24) & $7473(4)$ & $5070(3)$ & $-66(3)$ & $53(1)$ \\
\hline$C(25)$ & $8147(4)$ & $4402(3)$ & $2103(3)$ & $43(1)$ \\
\hline$C(26)$ & $7976(5)$ & $3419(3)$ & $1875(4)$ & $66(2)$ \\
\hline C (27) & $6950(4)$ & $8722(3)$ & $1015(3)$ & $49(1)$ \\
\hline C (28) & $7596(5)$ & $8667(4)$ & $363(4)$ & $64(2)$ \\
\hline$C(29)$ & $8778(4)$ & $8541(3)$ & $2492(3)$ & $47(1)$ \\
\hline$C(30)$ & $9162(4)$ & $8596(4)$ & $3494(3)$ & $59(1)$ \\
\hline$C(31)$ & $6560(4)$ & $8440(3)$ & $2671(3)$ & $43(1)$ \\
\hline C (32) & $6536(4)$ & $7903(4)$ & $3474(4)$ & $54(1)$ \\
\hline
\end{tabular}


Table 3. Distances [A] and angles [deg] for L2NiBr(corannulenyl)_1.

\begin{tabular}{|c|c|}
\hline $\mathrm{Br}(1)-\mathrm{Ni}(1)$ & $2.3551(7)$ \\
\hline $\mathrm{Ni}(1)-\mathrm{C}(1)$ & $1.880(4)$ \\
\hline $\mathrm{Ni}(1)-\mathrm{P}(2)$ & $2.2161(12)$ \\
\hline $\mathrm{Ni}(1)-\mathrm{P}(1)$ & $2.2210(11)$ \\
\hline$P(1)-C(23)$ & $1.808(4)$ \\
\hline$P(1)-C(25)$ & $1.833(4)$ \\
\hline$P(1)-C(21)$ & $1.836(4)$ \\
\hline$C(1)-C(2)$ & $1.429(6)$ \\
\hline$C(1)-C(11)$ & $1.446(6)$ \\
\hline$P(2)-C(29)$ & $1.819(4)$ \\
\hline$P(2)-C(31)$ & $1.821(5)$ \\
\hline$P(2)-C(27)$ & $1.843(5)$ \\
\hline$C(11)-C(16)$ & $1.392(6)$ \\
\hline$C(11)-C(10)$ & $1.424(6)$ \\
\hline$C(10)-C(9)$ & $1.400(6)$ \\
\hline$C(9)-C(15)$ & $1.433(7)$ \\
\hline$C(15)-C(20)$ & $1.391(6)$ \\
\hline$C(15)-C(8)$ & $1.431(6)$ \\
\hline$C(8)-C(7)$ & $1.415(7)$ \\
\hline$C(7)-C(14)$ & $1.414(7)$ \\
\hline$C(14)-C(19)$ & $1.392(6)$ \\
\hline$C(14)-C(6)$ & $1.420(7)$ \\
\hline$C(6)-C(5)$ & $1.419(7)$ \\
\hline$C(5)-C(13)$ & $1.435(6)$ \\
\hline$C(13)-C(18)$ & $1.377(6)$ \\
\hline$C(13)-C(4)$ & $1.412(7)$ \\
\hline$C(4)-C(3)$ & $1.433(7)$ \\
\hline$C(3)-C(12)$ & $1.411(6)$ \\
\hline$C(12)-C(17)$ & $1.397(6)$ \\
\hline$C(12)-C(2)$ & $1.437(6)$ \\
\hline$C(16)-C(17)$ & $1.383(6)$ \\
\hline$C(16)-C(20)$ & $1.405(6)$ \\
\hline$C(20)-C(19)$ & $1.389(6)$ \\
\hline $\mathrm{C}(19)-\mathrm{C}(18)$ & $1.410(6)$ \\
\hline$C(18)-C(17)$ & $1.408(6)$ \\
\hline$C(21)-C(22)$ & $1.504(7)$ \\
\hline$C(23)-C(24)$ & $1.512(6)$ \\
\hline$C(25)-C(26)$ & $1.532(6)$ \\
\hline$C(27)-C(28)$ & $1.468(7)$ \\
\hline$C(29)-C(30)$ & $1.509(7)$ \\
\hline$C(31)-C(32)$ & $1.499(7)$ \\
\hline $\mathrm{C}(1)-\mathrm{Ni}(1)-\mathrm{P}(2)$ & $92.87(13)$ \\
\hline $\mathrm{C}(1)-\mathrm{Ni}(1)-\mathrm{P}(1)$ & $89.05(12)$ \\
\hline $\mathrm{P}(2)-\mathrm{Ni}(1)-\mathrm{P}(1)$ & $176.49(5)$ \\
\hline $\mathrm{C}(1)-\mathrm{Ni}(1)-\mathrm{Br}(1)$ & $177.80(13)$ \\
\hline $\mathrm{P}(2)-\mathrm{Ni}(1)-\mathrm{Br}(1)$ & $89.27(4)$ \\
\hline $\mathrm{P}(1)-\mathrm{Ni}(1)-\mathrm{Br}(1)$ & $88.83(3)$ \\
\hline$C(23)-P(1)-C(25)$ & $104.6(2)$ \\
\hline$C(23)-P(1)-C(21)$ & $103.9(2)$ \\
\hline$C(25)-P(1)-C(21)$ & $103.9(2)$ \\
\hline $\mathrm{C}(23)-\mathrm{P}(1)-\mathrm{Ni}(1)$ & $112.74(15)$ \\
\hline $\mathrm{C}(25)-\mathrm{P}(1)-\mathrm{Ni}(1)$ & $118.52(14)$ \\
\hline $\mathrm{C}(21)-\mathrm{P}(1)-\mathrm{Ni}(1)$ & $111.82(16)$ \\
\hline
\end{tabular}




$\begin{array}{lc}C(2)-C(1)-C(11) & 119.1(4) \\ C(2)-C(1)-N i(1) & 120.7(3) \\ C(11)-C(1)-N i(1) & 120.2(3) \\ C(29)-P(2)-C(31) & 106.4(2) \\ C(29)-P(2)-C(27) & 100.9(2) \\ C(31)-P(2)-C(27) & 98.8(2) \\ C(29)-P(2)-N i(1) & 121.38(17) \\ C(31)-P(2)-N i(1) & 111.80(16) \\ C(27)-P(2)-N i(1) & 114.77(16) \\ C(16)-C(11)-C(10) & 114.1(4) \\ C(16)-C(11)-C(1) & 115.3(4) \\ C(10)-C(11)-C(1) & 130.0(4) \\ C(9)-C(10)-C(11) & 122.4(4) \\ C(10)-C(9)-C(15) & 122.5(4) \\ C(20)-C(15)-C(8) & 113.5(4) \\ C(20)-C(15)-C(9) & 113.4(4) \\ C(8)-C(15)-C(9) & 131.9(5) \\ C(7)-C(8)-C(15) & 122.1(5) \\ C(14)-C(7)-C(8) & 122.2(4) \\ C(19)-C(14)-C(7) & 113.4(5) \\ C(19)-C(14)-C(6) & 115.6(5) \\ C(7)-C(14)-C(6) & 129.7(4) \\ C(5)-C(6)-C(14) & 120.8(4) \\ C(6)-C(5)-C(13) & 122.7(5) \\ C(18)-C(13)-C(4) & 114.8(4) \\ C(18)-C(13)-C(5) & 113.6(5) \\ C(4)-C(13)-C(5) & 130.3(5) \\ C(13)-C(4)-C(3) & 121.5(4) \\ C(12)-C(3)-C(4) & 122.1(4) \\ C(17)-C(12)-C(3) & 114.2(4) \\ C(17)-C(12)-C(2) & 114.0(4) \\ C(3)-C(12)-C(2) & 130.7(4) \\ C(1)-C(2)-C(12) & 123.0(4) \\ C(17)-C(16)-C(11) & 123.9(4) \\ C(17)-C(16)-C(20) & 107.8(4) \\ C(11)-C(16)-C(20) & 122.8(4) \\ C(19)-C(20)-C(15) & 123.1(4) \\ C(19)-C(20)-C(16) & 108.3(4) \\ C(15)-C(20)-C(16) & 123.7(4) \\ C(20)-C(19)-C(14) & 124.1(5) \\ C(20)-C(19)-C(18) & 108.2(4) \\ C(14)-C(19)-C(18) & 122.1(4) \\ C(13)-C(18)-C(17) & 123.6(4) \\ C(13)-C(18)-C(19) & 124.1(4) \\ C(17)-C(18)-C(19) & 106.9(4) \\ C(16)-C(17)-C(12) & 122.8(4) \\ C(16)-C(17)-C(18) & 108.8(4) \\ C(12)-C(17)-C(18) & 122.5(4) \\ C(22)-C(21)-P(1) & 112.7(3) \\ C(24)-C(23)-P(1) & 113.7(3) \\ C(26)-C(25)-P(1) & 116.8(3) \\ C(28)-C(27)-P(2) & 114) \\ C(30)-C(29)-P(2) & \\ C(32)-C(31)-P(2) & \end{array}$


Table 4. Anisotropic displacement parameters ( $A^{\wedge} 2 \times 10^{\wedge} 3$ ) for L2NiBr (corannulenyl) 1.

The anisotropic displacement factor exponent takes the form:

$-2 \mathrm{pi}^{\wedge} 2\left[\mathrm{~h}^{\wedge} 2 \mathrm{a}^{\star \wedge} 2 \mathrm{U} 11+\ldots+2 \mathrm{~h} \mathrm{k} \mathrm{a}^{\star} \mathrm{b}\right.$ * U12]

\begin{tabular}{|c|c|c|c|c|c|c|}
\hline & $\mathrm{U} 11$ & $\mathrm{U} 22$ & U33 & U23 & $\mathrm{U} 13$ & $\mathrm{U} 12$ \\
\hline $\operatorname{Br}(1)$ & $23(1)$ & $52(1)$ & $56(1)$ & $0(1)$ & $3(1)$ & $2(1)$ \\
\hline $\mathrm{Ni}(1)$ & $16(1)$ & $30(1)$ & $32(1)$ & $-3(1)$ & $7(1)$ & $-4(1)$ \\
\hline$P(1)$ & $21(1)$ & $32(1)$ & $37(1)$ & $-3(1)$ & $8(1)$ & $-9(1)$ \\
\hline$C(1)$ & $22(2)$ & $34(2)$ & $50(3)$ & $-4(2)$ & $9(2)$ & $-7(2)$ \\
\hline $\mathrm{P}(2)$ & $35(1)$ & $33(1)$ & $44(1)$ & $-6(1)$ & $18(1)$ & $-5(1)$ \\
\hline$C(11)$ & $29(2)$ & $35(2)$ & $40(2)$ & $-9(2)$ & $10(2)$ & $-9(2)$ \\
\hline$C(10)$ & $31(2)$ & $42(2)$ & $34(2)$ & $-8(2)$ & $2(2)$ & $-4(2)$ \\
\hline$C(9)$ & $39(3)$ & $51(3)$ & $36(3)$ & $-11(2)$ & $13(2)$ & $-8(2)$ \\
\hline$C(15)$ & $32(2)$ & $41(2)$ & $49(3)$ & $-17(2)$ & $18(2)$ & $-3(2)$ \\
\hline C ( 8) & $38(3)$ & $43(3)$ & $60(3)$ & $-8(2)$ & $27(2)$ & $-4(2)$ \\
\hline$C(7)$ & $29(2)$ & $48(3)$ & $62(3)$ & $-2(2)$ & $21(2)$ & $3(2)$ \\
\hline$C(14)$ & $31(2)$ & $42(2)$ & $60(3)$ & $-1(2)$ & $17(2)$ & $8(2)$ \\
\hline$C(6)$ & $22(2)$ & $49(3)$ & $68(4)$ & $-2(2)$ & $1(2)$ & $2(2)$ \\
\hline$C(5)$ & $30(2)$ & $49(3)$ & $61(3)$ & $4(2)$ & $-3(2)$ & $3(2)$ \\
\hline$C(13)$ & $31(2)$ & $40(2)$ & $53(3)$ & $5(2)$ & $4(2)$ & $2(2)$ \\
\hline C (4) & $47(3)$ & $54(3)$ & $33(3)$ & $4(2)$ & $4(2)$ & $1(2)$ \\
\hline$C(3)$ & $42(3)$ & $56(3)$ & $44(3)$ & $4(2)$ & $18(2)$ & $-2(2)$ \\
\hline$C(12)$ & $34(2)$ & $39(2)$ & $44(3)$ & $1(2)$ & $10(2)$ & $-7(2)$ \\
\hline C (2) & $24(2)$ & $40(2)$ & $48(3)$ & $-5(2)$ & $15(2)$ & $-6(2)$ \\
\hline$C(16)$ & $27(2)$ & $26(2)$ & $47(3)$ & $-9(2)$ & $6(2)$ & $-4(2)$ \\
\hline$C(20)$ & $44(3)$ & $29(2)$ & $43(3)$ & $-4(2)$ & $22(2)$ & $3(2)$ \\
\hline$C(19)$ & $18(2)$ & $25(2)$ & $72(3)$ & $-7(2)$ & $12(2)$ & $2(1)$ \\
\hline$C(18)$ & $36(2)$ & $26(2)$ & $40(3)$ & $3(2)$ & $3(2)$ & $4(2)$ \\
\hline$C(17)$ & $31(2)$ & $27(2)$ & $56(3)$ & $-1(2)$ & $21(2)$ & $-4(2)$ \\
\hline$C(21)$ & $32(2)$ & $48(3)$ & $60(3)$ & $-2(2)$ & $16(2)$ & $-17(2)$ \\
\hline$C(22)$ & $41(3)$ & $68(3)$ & $58(3)$ & $10(3)$ & $23(3)$ & $-7(2)$ \\
\hline$C(23)$ & $40(3)$ & $43(2)$ & $42(3)$ & $-12(2)$ & $8(2)$ & $-7(2)$ \\
\hline$C(24)$ & $61(3)$ & $61(3)$ & $39(3)$ & $-11(2)$ & $19(3)$ & $-6(2)$ \\
\hline$C(25)$ & $36(2)$ & $31(2)$ & $62(3)$ & $9(2)$ & $14(2)$ & $-2(2)$ \\
\hline$C(26)$ & $59(3)$ & $34(3)$ & $101(5)$ & $8(3)$ & $14(3)$ & $-6(2)$ \\
\hline$C(27)$ & $41(3)$ & $36(2)$ & $66(3)$ & $1(2)$ & $6(2)$ & $-1(2)$ \\
\hline$C(28)$ & $86(4)$ & $56(3)$ & $50(3)$ & $6(3)$ & $20(3)$ & $13(3)$ \\
\hline C (29) & $34(2)$ & $46(3)$ & $61(3)$ & $-5(2)$ & $13(2)$ & $-9(2)$ \\
\hline$C(30)$ & $37(3)$ & $90(4)$ & $46(3)$ & $10(3)$ & $3(2)$ & $-3(3)$ \\
\hline$C(31)$ & $36(2)$ & $43(2)$ & $49(3)$ & $-9(2)$ & $9(2)$ & $8(2)$ \\
\hline$C(32)$ & $42(3)$ & $67(3)$ & $60(3)$ & $-11(3)$ & $28(3)$ & $-5(2)$ \\
\hline
\end{tabular}


Table 4. Crystal data and structure refinement for transPt (PEt3) 2 (Br) (corannulenyl) 2 .

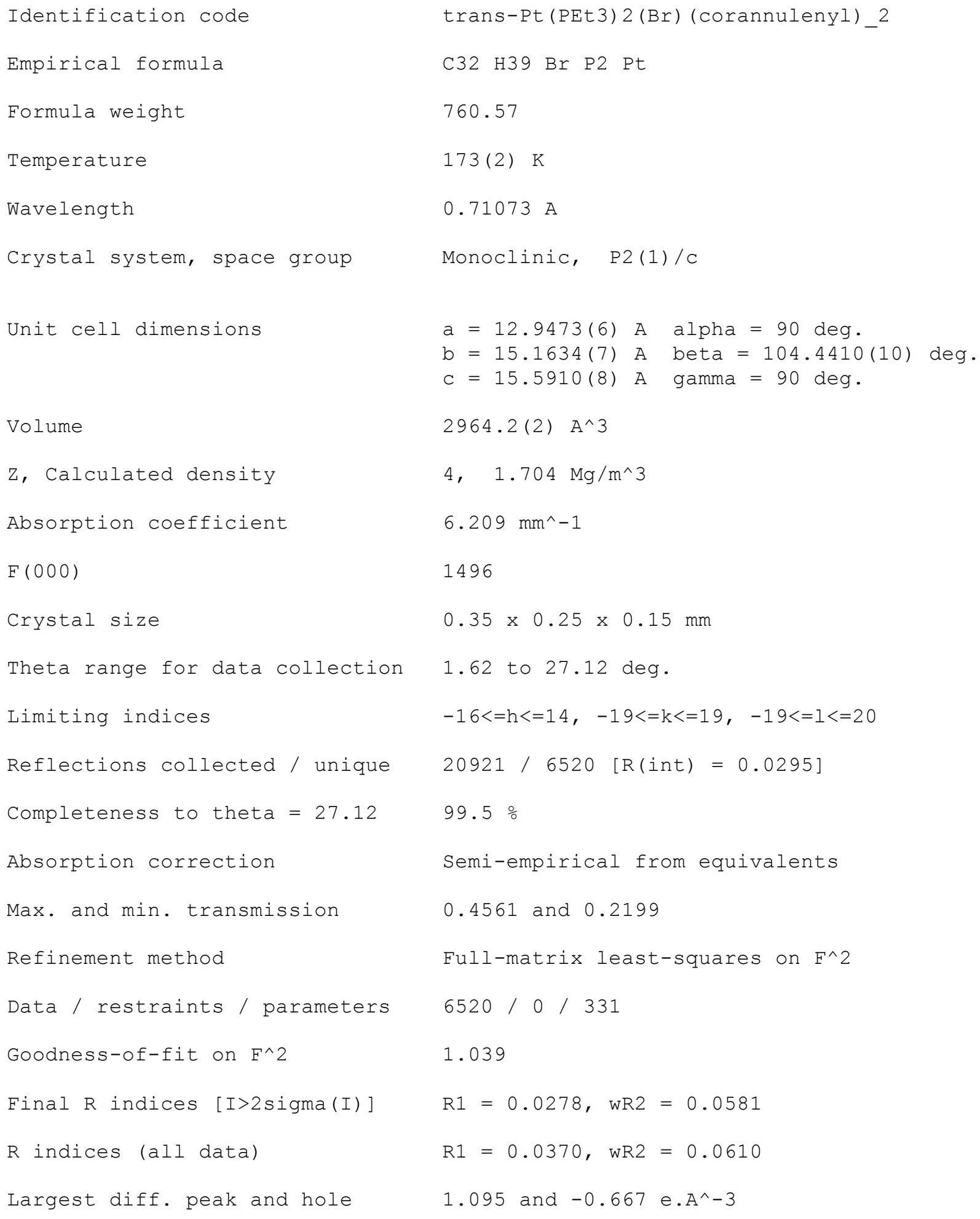


Table 5. Atomic coordinates $\left(x 10^{\wedge} 4\right)$ and equivalent isotropic displacement parameters $\left(A^{\wedge} 2 \times 10^{\wedge} 3\right)$ for transPt (PEt3) 2 (Br) (corannulenyl) 2 .

$\mathrm{U}(\mathrm{eq})$ is defined as one third of the trace of the orthogonalized Uij tensor.

\begin{tabular}{|c|c|c|c|c|}
\hline & $\mathrm{x}$ & y & z & $\mathrm{U}(\mathrm{eq})$ \\
\hline Pt (1) & 7307 (1) & $1583(1)$ & $1794(1)$ & $23(1)$ \\
\hline $\operatorname{Br}(1)$ & $5416(1)$ & $1800(1)$ & $893(1)$ & $42(1)$ \\
\hline P (1) & $7053(1)$ & $83(1)$ & $1585(1)$ & $31(1)$ \\
\hline P (2) & $7427(1)$ & $3087(1)$ & $2007(1)$ & $30(1)$ \\
\hline C (1) & $8802(3)$ & $1356(3)$ & $2509(3)$ & $32(1)$ \\
\hline C (11) & $9661(3)$ & $1269(3)$ & $2062(3)$ & $33(1)$ \\
\hline$C(10)$ & $9732(3)$ & $1590(3)$ & $1218(3)$ & $35(1)$ \\
\hline C (9) & $10711(4)$ & $1675(3)$ & $985(3)$ & $41(1)$ \\
\hline C (15) & $11705(4)$ & $1454(3)$ & $1586(3)$ & $40(1)$ \\
\hline C (8) & $12778(4)$ & $1712(3)$ & $1618(3)$ & $44(1)$ \\
\hline$C(7)$ & $13599(4)$ & $1675(3)$ & $2413(3)$ & $47(1)$ \\
\hline C (14) & $13414(3)$ & $1376(3)$ & $3220(3)$ & $43(1)$ \\
\hline C (6) & $13971(4)$ & $1536(3)$ & $4108(3)$ & $49(1)$ \\
\hline$C(5)$ & $13491(4)$ & $1428(3)$ & $4827(3)$ & $49(1)$ \\
\hline C (13) & $12414(4)$ & $1150(3)$ & $4700(3)$ & $43(1)$ \\
\hline C ( 4$)$ & $11686(4)$ & 1251 (3) & $5239(3)$ & $47(1)$ \\
\hline C (3) & $10575(4)$ & $1197(3)$ & $4893(3)$ & $46(1)$ \\
\hline$C(12)$ & $10094(3)$ & $1062(3)$ & $3977(3)$ & $36(1)$ \\
\hline$C(2)$ & $9048(3)$ & $1250(3)$ & $3440(3)$ & $33(1)$ \\
\hline$C(16)$ & $10614(3)$ & $932(2)$ & $2579(3)$ & $34(1)$ \\
\hline C (20) & $11589(4)$ & $1023(3)$ & $2341(3)$ & $36(1)$ \\
\hline C (19) & $12412(3)$ & $992(3)$ & $3126(3)$ & $38(1)$ \\
\hline C (18) & $11937(3)$ & $878(3)$ & $3836(3)$ & $37(1)$ \\
\hline C (17) & $10831(3)$ & $833(2)$ & $3496(3)$ & $34(1)$ \\
\hline$C(21)$ & $5949(4)$ & $-314(3)$ & $2023(3)$ & $50(1)$ \\
\hline C (22) & $6047(4)$ & $-32(4)$ & $2974(3)$ & $56(1)$ \\
\hline C (23) & $6668(4)$ & $-220(3)$ & 425 (3) & $46(1)$ \\
\hline C (24) & $7498(5)$ & $51(3)$ & $-63(3)$ & $61(1)$ \\
\hline C (25) & $8175(4)$ & $-629(3)$ & $2085(3)$ & $43(1)$ \\
\hline$C(26)$ & $8055(5)$ & $-1617(3)$ & $1876(4)$ & $68(2)$ \\
\hline C (27) & $6946(4)$ & $3731(3)$ & $992(3)$ & $42(1)$ \\
\hline C (28) & $7623(5)$ & $3663(3)$ & $347(3)$ & $61(2)$ \\
\hline C (29) & $8738(3)$ & $3579(3)$ & $2484(3)$ & $40(1)$ \\
\hline$C(30)$ & $9119(4)$ & $3607(3)$ & $3490(3)$ & $53(1)$ \\
\hline C (31) & $6542(4)$ & $3478(3)$ & $2668(3)$ & $42(1)$ \\
\hline$C(32)$ & $6529(4)$ & $2934(3)$ & 3477 (3) & $51(1)$ \\
\hline
\end{tabular}


Table 6. Distances [A] and angles [deg] for transPt (PEt3) 2 (Br) (corannulenyl) 2 .

\begin{tabular}{|c|c|}
\hline Pt (1) $-\mathrm{C}(1)$ & $2.008(4)$ \\
\hline Pt (1) $-P(2)$ & $2.3042(10)$ \\
\hline $\operatorname{Pt}(1)-\mathrm{P}(1)$ & $2.3103(10)$ \\
\hline $\operatorname{Pt}(1)-\operatorname{Br}(1)$ & $2.5213(4)$ \\
\hline$P(1)-C(23)$ & $1.812(4)$ \\
\hline$P(1)-C(25)$ & $1.822(4)$ \\
\hline$P(1)-C(21)$ & $1.833(4)$ \\
\hline$P(2)-C(31)$ & $1.821(4)$ \\
\hline$P(2)-C(27)$ & $1.832(4)$ \\
\hline$P(2)-C(29)$ & $1.833(4)$ \\
\hline$C(1)-C(2)$ & $1.416(5)$ \\
\hline$C(1)-C(11)$ & $1.458(6)$ \\
\hline$C(11)-C(16)$ & $1.392(6)$ \\
\hline$C(11)-C(10)$ & $1.428(6)$ \\
\hline$C(10)-C(9)$ & $1.408(6)$ \\
\hline$C(9)-C(15)$ & $1.430(6)$ \\
\hline$C(15)-C(20)$ & $1.386(6)$ \\
\hline$C(15)-C(8)$ & $1.433(6)$ \\
\hline$C(8)-C(7)$ & $1.418(7)$ \\
\hline$C(7)-C(14)$ & $1.413(7)$ \\
\hline$C(14)-C(19)$ & $1.397(6)$ \\
\hline$C(14)-C(6)$ & $1.413(7)$ \\
\hline$C(6)-C(5)$ & $1.421(7)$ \\
\hline$C(5)-C(13)$ & $1.422(6)$ \\
\hline$C(13)-C(18)$ & $1.396(6)$ \\
\hline C (13) -C (4) & $1.420(7)$ \\
\hline$C(4)-C(3)$ & $1.406(6)$ \\
\hline$C(3)-C(12)$ & $1.424(6)$ \\
\hline$C(12)-C(17)$ & $1.397(6)$ \\
\hline$C(12)-C(2)$ & $1.432(6)$ \\
\hline$C(16)-C(17)$ & $1.394(6)$ \\
\hline$C(16)-C(20)$ & $1.408(6)$ \\
\hline$C(20)-C(19)$ & $1.408(6)$ \\
\hline$C(19)-C(18)$ & $1.405(6)$ \\
\hline$C(18)-C(17)$ & $1.399(6)$ \\
\hline$C(21)-C(22)$ & $1.518(7)$ \\
\hline$C(23)-C(24)$ & $1.519(7)$ \\
\hline$C(25)-C(26)$ & $1.532(6)$ \\
\hline$C(27)-C(28)$ & $1.494(7)$ \\
\hline$C(29)-C(30)$ & $1.524(6)$ \\
\hline$C(31)-C(32)$ & $1.509(6)$ \\
\hline $\mathrm{C}(1)-\mathrm{Pt}(1)-\mathrm{P}(2)$ & $93.94(11)$ \\
\hline $\mathrm{C}(1)-\mathrm{Pt}(1)-\mathrm{P}(1)$ & $89.56(11)$ \\
\hline $\mathrm{P}(2)-\mathrm{Pt}(1)-\mathrm{P}(1)$ & $175.66(4)$ \\
\hline $\mathrm{C}(1)-\operatorname{Pt}(1)-\mathrm{Br}(1)$ & $177.59(11)$ \\
\hline $\mathrm{P}(2)-\operatorname{Pt}(1)-\operatorname{Br}(1)$ & $88.45(3)$ \\
\hline $\mathrm{P}(1)-\operatorname{Pt}(1)-\operatorname{Br}(1)$ & $88.04(3)$ \\
\hline$C(23)-P(1)-C(25)$ & $105.6(2)$ \\
\hline$C(23)-P(1)-C(21)$ & $104.0(2)$ \\
\hline$C(25)-P(1)-C(21)$ & $105.0(2)$ \\
\hline $\mathrm{C}(23)-\mathrm{P}(1)-\mathrm{Pt}(1)$ & $112.57(15)$ \\
\hline
\end{tabular}




$\begin{array}{ll}C(25)-P(1)-P t(1) & 116.98(14) \\ C(21)-P(1)-P t(1) & 111.57(16) \\ C(31)-P(2)-C(27) & 100.9(2) \\ C(31)-P(2)-C(29) & 106.7(2) \\ C(27)-P(2)-C(29) & 102.4(2) \\ C(31)-P(2)-P t(1) & 112.00(15) \\ C(27)-P(2)-P t(1) & 113.99(15) \\ C(29)-P(2)-P t(1) & 118.91(15) \\ C(2)-C(1)-C(11) & 118.4(4) \\ C(2)-C(1)-P t(1) & 121.9(3) \\ C(11)-C(1)-P t(1) & 119.7(3) \\ C(16)-C(11)-C(10) & 114.0(4) \\ C(16)-C(11)-C(1) & 115.4(4) \\ C(10)-C(11)-C(1) & 129.9(4) \\ C(9)-C(10)-C(11) & 122.6(4) \\ C(10)-C(9)-C(15) & 122.0(4) \\ C(20)-C(15)-C(9) & 113.4(4) \\ C(20)-C(15)-C(8) & 114.1(4) \\ C(9)-C(15)-C(8) & 131.7(4) \\ C(7)-C(8)-C(15) & 121.9(4) \\ C(14)-C(7)-C(8) & 122.3(4) \\ C(19)-C(14)-C(7) & 113.5(4) \\ C(19)-C(14)-C(6) & 114.1(5) \\ C(7)-C(14)-C(6) & 131.2(5) \\ C(14)-C(6)-C(5) & 122.5(4) \\ C(6)-C(5)-C(13) & 122.0(4) \\ C(18)-C(13)-C(4) & 113.8(4) \\ C(18)-C(13)-C(5) & 113.9(4) \\ C(4)-C(13)-C(5) & 131.1(4) \\ C(3)-C(4)-C(13) & 122.2(4) \\ C(4)-C(3)-C(12) & 122.9(4) \\ C(17)-C(12)-C(3) & 112.8(4) \\ C(17)-C(12)-C(2) & 114.1(4) \\ C(3)-C(12)-C(2) & 132.2(4) \\ C(1)-C(2)-C(12) & 124.1(4) \\ C(11)-C(16)-C(17) & 123.8(4) \\ C(11)-C(16)-C(20) & 122.3(4) \\ C(17)-C(16)-C(20) & 108.4(4) \\ C(15)-C(20)-C(19) & 122.3(4) \\ C(15)-C(20)-C(16) & 124.3(4) \\ C(19)-C(20)-C(16) & 107.5(4) \\ C(14)-C(19)-C(18) & 123.1(4) \\ C(14)-C(19)-C(20) & 124.0(4) \\ C(18)-C(19)-C(20) & 107.7(3) \\ C(13)-C(18)-C(17) & 123.0(4) \\ C(13)-C(18)-C(19) & 123.3(4) \\ C(17)-C(18)-C(19) & 108.3(4) \\ C(16)-C(17)-C(12) & 122.2(4) \\ C(16)-C(17)-C(18) & 108.0(4) \\ C(12)-C(17)-C(18) & 123.8(4) \\ C(22)-C(21)-P(1) & 112.8(3) \\ C(24)-C(23)-P(1) & 112.3(3) \\ C(26)-C(25)-P(1) & \\ C(28)-C(27)-P(2) & \\ C(30)-C(29)-P(2) & \\ C(32)-C(31)-P(2) & \end{array}$


Table 7. Anisotropic displacement parameters $\left(A^{\wedge} 2 \times 10^{\wedge} 3\right)$ for transPt (PEt3) 2 (Br) (corannulenyl) 2.

The anisotropic displacement factor exponent takes the form:

$-2 \mathrm{pi}^{\wedge} 2\left[\mathrm{~h}^{\wedge} 2 \mathrm{a}^{\star \wedge} 2 \mathrm{U} 11+\ldots+2 \mathrm{~h} \mathrm{k} \mathrm{a}^{\star} \mathrm{b}^{\star} \mathrm{U} 12\right.$ ]

\begin{tabular}{|c|c|c|c|c|c|c|}
\hline & $\mathrm{U} 11$ & U22 & U33 & U23 & U13 & $\mathrm{U} 12$ \\
\hline Pt (1) & $17(1)$ & $25(1)$ & $27(1)$ & $-2(1)$ & $4(1)$ & $-3(1)$ \\
\hline $\operatorname{Br}(1)$ & $20(1)$ & $49(1)$ & $51(1)$ & $0(1)$ & $0(1)$ & $1(1)$ \\
\hline P (1) & $26(1)$ & $28(1)$ & $36(1)$ & $-2(1)$ & $5(1)$ & $-8(1)$ \\
\hline P (2) & $29(1)$ & 27 (1) & $33(1)$ & $-3(1)$ & $8(1)$ & $0(1)$ \\
\hline$C(1)$ & $30(2)$ & $26(2)$ & $40(2)$ & $-4(2)$ & $9(2)$ & $-4(2)$ \\
\hline$C(11)$ & $33(2)$ & $29(2)$ & $36(2)$ & $-6(2)$ & $6(2)$ & $-5(2)$ \\
\hline$C(10)$ & $31(2)$ & $36(2)$ & $34(2)$ & $-6(2)$ & $3(2)$ & $-3(2)$ \\
\hline$C(9)$ & $43(3)$ & $44(3)$ & $36(2)$ & $-2(2)$ & $13(2)$ & $-1(2)$ \\
\hline$C(15)$ & $34(2)$ & $46(3)$ & $45(2)$ & $-13(2)$ & $15(2)$ & $0(2)$ \\
\hline$C(8)$ & $35(2)$ & $44(3)$ & $58(3)$ & $-12(2)$ & $23(2)$ & $-3(2)$ \\
\hline$C(7)$ & $35(2)$ & $46(3)$ & $66(3)$ & $-7(2)$ & $26(2)$ & $0(2)$ \\
\hline$C(14)$ & $29(2)$ & $40(2)$ & $57(3)$ & $-1(2)$ & $6(2)$ & $7(2)$ \\
\hline$C(6)$ & $25(2)$ & $47(3)$ & $64(3)$ & $-3(2)$ & $-6(2)$ & $4(2)$ \\
\hline C (5) & $33(2)$ & $57(3)$ & $48(3)$ & $3(2)$ & $-11(2)$ & $4(2)$ \\
\hline$C(13)$ & $41(3)$ & $40(3)$ & $42(2)$ & $5(2)$ & $-2(2)$ & $4(2)$ \\
\hline$C(4)$ & $47(3)$ & $58(3)$ & $32(2)$ & $6(2)$ & $2(2)$ & $4(2)$ \\
\hline C (3) & $43(3)$ & $55(3)$ & $39(2)$ & $5(2)$ & $11(2)$ & $-2(2)$ \\
\hline$C(12)$ & $35(2)$ & $34(2)$ & $39(2)$ & $7(2)$ & $8(2)$ & $-5(2)$ \\
\hline$C(2)$ & $28(2)$ & $34(2)$ & $39(2)$ & $-1(2)$ & $12(2)$ & $-6(2)$ \\
\hline$C(16)$ & $32(2)$ & $21(2)$ & $46(2)$ & $-9(2)$ & $2(2)$ & $-2(2)$ \\
\hline$C(20)$ & $47(3)$ & $25(2)$ & $38(2)$ & $-6(2)$ & $15(2)$ & $3(2)$ \\
\hline$C(19)$ & $21(2)$ & $23(2)$ & $68(3)$ & $-5(2)$ & $10(2)$ & $5(2)$ \\
\hline$C(18)$ & $38(2)$ & $28(2)$ & $39(2)$ & $4(2)$ & $-1(2)$ & $5(2)$ \\
\hline$C(17)$ & $35(2)$ & $23(2)$ & $47(2)$ & $1(2)$ & $16(2)$ & $-3(2)$ \\
\hline$C(21)$ & $39(3)$ & $45(3)$ & $68(3)$ & $4(2)$ & $18(2)$ & $-15(2)$ \\
\hline C (22) & $47(3)$ & $68(3)$ & $58(3)$ & $13(3)$ & $22(2)$ & $-8(3)$ \\
\hline$C(23)$ & $50(3)$ & $40(2)$ & $43(2)$ & $-15(2)$ & $0(2)$ & $-9(2)$ \\
\hline C (24) & $88(4)$ & $54(3)$ & $46(3)$ & $-13(2)$ & $25(3)$ & $-1(3)$ \\
\hline$C(25)$ & $43(3)$ & $27(2)$ & $58(3)$ & $4(2)$ & $11(2)$ & $-4(2)$ \\
\hline$C(26)$ & $63(4)$ & $30(3)$ & $106(5)$ & $7(3)$ & $14(3)$ & $-6(2)$ \\
\hline$C(27)$ & $42(3)$ & $33(2)$ & $47(3)$ & $4(2)$ & $5(2)$ & $-3(2)$ \\
\hline$C(28)$ & $87(4)$ & $55(3)$ & $43(3)$ & $6(2)$ & $24(3)$ & $9(3)$ \\
\hline$C(29)$ & $35(2)$ & $33(2)$ & $49(3)$ & $-6(2)$ & $5(2)$ & $-5(2)$ \\
\hline$C(30)$ & $44(3)$ & $63(3)$ & $46(3)$ & $-1(2)$ & $-1(2)$ & $-9(2)$ \\
\hline$C(31)$ & $37(2)$ & $44(3)$ & $46(2)$ & $-7(2)$ & $12(2)$ & $11(2)$ \\
\hline$C(32)$ & $53(3)$ & $57(3)$ & $50(3)$ & $-7(2)$ & $26(2)$ & $-1(2)$ \\
\hline
\end{tabular}


Table 8. Crystal data and structure refinement for trans-3/trans-4.

Identification code

Empirical formula

Formula weight

Temperature

Wavelength

Crystal system, space group

Unit cell dimensions

Volume

Z, Calculated density

Absorption coefficient

$F(000)$

Crystal size

Theta range for data collection

Limiting indices

Reflections collected / unique

Completeness to theta $=24.89$

Absorption correction

Max. and min. transmission

Refinement method

Data / restraints / parameters

Goodness-of-fit on $\mathrm{F}^{\wedge} 2$

Final $\mathrm{R}$ indices [I>2sigma(I)]

$\mathrm{R}$ indices (all data)

Largest diff. peak and hole

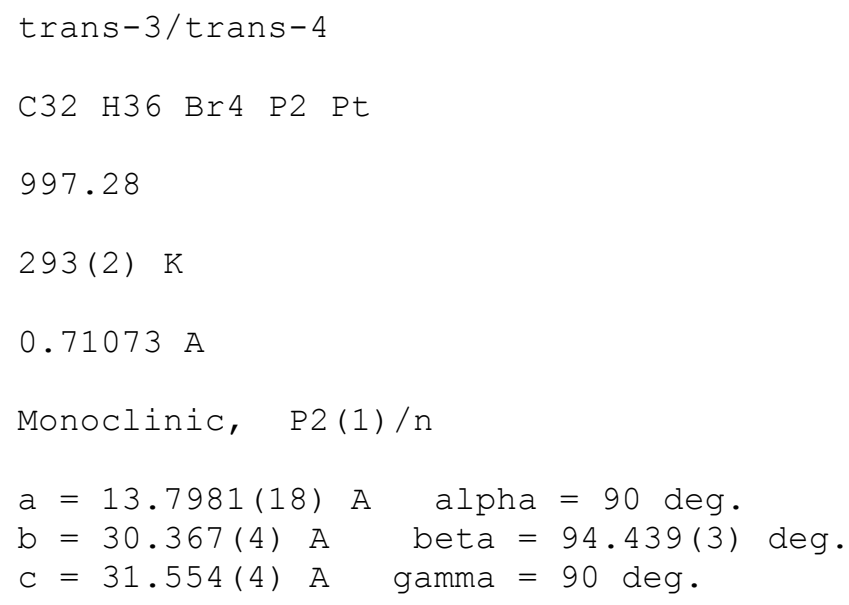


Table 9. Atomic coordinates ( $x$ 10^4) and equivalent isotropic displacement parameters $\left(A^{\wedge} 2 \times 10^{\wedge} 3\right)$ for trans-3/trans 4 . $\mathrm{U}(\mathrm{eq})$ is defined as one third of the trace of the orthogonalized Uij tensor.

\begin{tabular}{|c|c|c|c|c|}
\hline & $\mathrm{x}$ & $\mathrm{Y}$ & z & $\mathrm{U}(\mathrm{eq})$ \\
\hline Pt (1A) & $5432(1)$ & $2095(1)$ & $3677(1)$ & $34(1)$ \\
\hline Pt (1B) & $7469(1)$ & $2838(1)$ & $5996(1)$ & $43(1)$ \\
\hline Pt (1C) & $4579(1)$ & $5228(1)$ & $1192(1)$ & $43(1)$ \\
\hline Pt (1D) & $7379(1)$ & $5146(1)$ & $3847(1)$ & $47(1)$ \\
\hline$P(1 A)$ & $6821(4)$ & $2506(2)$ & $3813(2)$ & $41(2)$ \\
\hline$P(2 A)$ & $4177(5)$ & $1620(2)$ & $3480(2)$ & $43(2)$ \\
\hline$P(1 B)$ & $8821(5)$ & $2422(2)$ & $5913(2)$ & $47(2)$ \\
\hline$P(2 B)$ & $6165(6)$ & $3281(3)$ & $6124(2)$ & $68(2)$ \\
\hline$P(1 C)$ & $3144(5)$ & $4846(2)$ & $1083(2)$ & $51(2)$ \\
\hline$P(2 C)$ & $5929(6)$ & $5654(3)$ & $1374(3)$ & $71(2)$ \\
\hline$P(1 D)$ & $6011(5)$ & $4725(3)$ & $3919(2)$ & $63(2)$ \\
\hline$P(2 D)$ & $8683(6)$ & $5607(2)$ & $3774(2)$ & $65(2)$ \\
\hline$C(21 A)$ & $6787(15)$ & $3052(7)$ & $3590(7)$ & $41(6)$ \\
\hline$C(22 A)$ & $7794(17)$ & $3289(8)$ & $3625(7)$ & $53(7)$ \\
\hline$C(23 A)$ & $7250(20)$ & $2578(10)$ & $4370(8)$ & $84(10)$ \\
\hline$C(24 A)$ & $6740(20)$ & $2908(10)$ & $4624(8)$ & $94(11)$ \\
\hline$C(25 A)$ & $7847(16)$ & $2239(8)$ & $3594(10)$ & $66(9)$ \\
\hline$C(26 A)$ & $7700(20)$ & $2168(10)$ & $3120(9)$ & $90(11)$ \\
\hline$C(27 A)$ & $3720(18)$ & $1336(9)$ & $3912(8)$ & $62(8)$ \\
\hline$C(28 A)$ & $3400(20)$ & $1619(10)$ & $4292(8)$ & $89(11)$ \\
\hline$C(29 A)$ & $3120(16)$ & $1870(8)$ & $3169(8)$ & $60(8)$ \\
\hline$C(30 A)$ & $2338(17)$ & $1532(8)$ & $2989(8)$ & $65(9)$ \\
\hline$C(31 A)$ & $4580(20)$ & 1171 ( 8) & $3137(9)$ & $77(10)$ \\
\hline$C(32 A)$ & $5000(20)$ & $1306(12)$ & $2723(8)$ & 115 (15) \\
\hline$C(21 B)$ & $9929(18)$ & $2724(9)$ & $6108(10)$ & $75(10)$ \\
\hline$C(22 B)$ & $9930(20)$ & $2879(11)$ & $6576(10)$ & $112(14)$ \\
\hline$C(23 B)$ & $8938(14)$ & $1907(7)$ & $6225(7)$ & $37(6)$ \\
\hline$C(24 B)$ & $9916(15)$ & $1661(8)$ & $6208(7)$ & $53(7)$ \\
\hline$C(25 B)$ & $9050(20)$ & $2288(9)$ & $5353(8)$ & $79(10)$ \\
\hline$C(26 B)$ & $8650(30)$ & $1858(11)$ & $5173(9)$ & $129(16)$ \\
\hline$C(27 B)$ & $5230(20)$ & 3315 (11) & $5617(9)$ & $101(11)$ \\
\hline$C(28 B)$ & $5630(30)$ & $3634(12)$ & $5356(17)$ & $220(30)$ \\
\hline$C(29 B)$ & $6520(30)$ & $3814(10)$ & $6295(11)$ & $109(13)$ \\
\hline$C(30 B)$ & $7110(20)$ & $3803(10)$ & $6712(11)$ & $106(13)$ \\
\hline$C(31 B)$ & $5390(20)$ & $3056(10)$ & $6531(10)$ & $98(12)$ \\
\hline$C(32 B)$ & $4650(30)$ & 3395 (11) & $6683(10)$ & $124(14)$ \\
\hline$C(21 C)$ & $3210(20)$ & 4275 (11) & $1041(15)$ & $160(20)$ \\
\hline$C(22 C)$ & $2150(30)$ & $4038(12)$ & $1005(12)$ & $146(16)$ \\
\hline$C(23 C)$ & $2250(30)$ & $5041(16)$ & $697(16)$ & $230(30)$ \\
\hline$C(24 C)$ & $2550(20)$ & $5066(15)$ & $251(10)$ & $149(19)$ \\
\hline$C(25 C)$ & $2400(40)$ & $4875(17)$ & $1580(15)$ & $200(20)$ \\
\hline$C(26 C)$ & $2520(30)$ & $5268(11)$ & $1818(10)$ & $130(16)$ \\
\hline$C(27 C)$ & $5690(30)$ & $6111(10)$ & $1727(13)$ & 131 (19) \\
\hline$C(28 C)$ & $5340(30)$ & $5979(13)$ & $2159(12)$ & $132(17)$ \\
\hline$C(29 C)$ & $6943(18)$ & $5378(8)$ & $1670(9)$ & $69(9)$ \\
\hline$C(30 C)$ & $7741(17)$ & $5669(9)$ & $1857(9)$ & $78(9)$ \\
\hline
\end{tabular}




\begin{tabular}{|c|c|c|c|c|}
\hline$C(31 C)$ & $6330(30)$ & $5934(14)$ & $940(14)$ & $160(20)$ \\
\hline$C(32 C)$ & $6570(30)$ & $5678(14)$ & $574(11)$ & 142 (19) \\
\hline$C(21 D)$ & $5820(60)$ & $4520(20)$ & $4467(15)$ & $370(60)$ \\
\hline$C(22 D)$ & $6090(30)$ & $4831(15)$ & $4739(17)$ & $240(30)$ \\
\hline$C(23 D)$ & $4876(19)$ & 4991 (11) & $3748(12)$ & $110(14)$ \\
\hline$C(24 D)$ & $4920(30)$ & $5173(13)$ & $3272(13)$ & $200(30)$ \\
\hline$C(25 D)$ & $6000(20)$ & $4206(8)$ & $3694(12)$ & $104(13)$ \\
\hline$C(26 D)$ & $4990(20)$ & $3944(10)$ & $3683(10)$ & $106(12)$ \\
\hline$C(27 D)$ & $9210(20)$ & $5803(10)$ & $4306(9)$ & $87(10)$ \\
\hline$C(28 D)$ & $9420(20)$ & $5424(11)$ & $4596(12)$ & $128(15)$ \\
\hline$C(29 D)$ & $8360(20)$ & $6139(9)$ & $3504(11)$ & $99(12)$ \\
\hline$C(30 D)$ & $7840(20)$ & $6055(10)$ & $3068(10)$ & $103(12)$ \\
\hline$C(31 D)$ & $9660(19)$ & 5402 ( 8) & 3485 (9) & $70(9)$ \\
\hline$C(32 D)$ & $10490(20)$ & $5726(11)$ & $3401(10)$ & $117(14)$ \\
\hline $\operatorname{Br}(1 \mathrm{~A})$ & $5442(8)$ & $2496(3)$ & $2638(4)$ & $49(2)$ \\
\hline $\operatorname{Br}(2 \mathrm{~A})$ & $6283(18)$ & $1448(10)$ & $4069(6)$ & $35(2)$ \\
\hline $\operatorname{Br}(5 A)$ & 2772 ( 3 ) & $4006(2)$ & $4901(1)$ & $72(2)$ \\
\hline $\operatorname{Br}(6 A)$ & $2063(5)$ & $4807(2)$ & $4222(2)$ & $80(2)$ \\
\hline $\operatorname{Br}(1 B)$ & $8334(13)$ & $3493(5)$ & $5692(6)$ & $55(3)$ \\
\hline $\mathrm{Br}(2 \mathrm{~B})$ & $7539(14)$ & $2478(8)$ & $7031(7)$ & $51(4)$ \\
\hline $\operatorname{Br}(5 B)$ & $5024(3)$ & $104(1)$ & $7097(1)$ & $67(1)$ \\
\hline $\operatorname{Br}(6 B)$ & $4367(3)$ & $-192(1)$ & $6094(1)$ & $63(1)$ \\
\hline $\mathrm{Br}(1 \mathrm{C})$ & 3761 (9) & $5956(4)$ & $1019(3)$ & $61(2)$ \\
\hline $\mathrm{Br}(2 \mathrm{C})$ & $5056(11)$ & $4741(8)$ & $2173(5)$ & $65(3)$ \\
\hline $\operatorname{Br}(5 C)$ & $7683(3)$ & $2433(2)$ & $1934(1)$ & $83(2)$ \\
\hline $\mathrm{Br}(6 \mathrm{C})$ & $7740(3)$ & $2214(2)$ & $912(2)$ & $77(2)$ \\
\hline $\operatorname{Br}(1 \mathrm{D})$ & $6522(2)$ & $5801(1)$ & $4121(1)$ & $87(1)$ \\
\hline $\mathrm{Br}(2 \mathrm{D})$ & $7455(2)$ & $4809(1)$ & $2800(1)$ & $55(1)$ \\
\hline $\operatorname{Br}(5 \mathrm{D})$ & $10217(3)$ & $2489(1)$ & $2727(1)$ & $75(1)$ \\
\hline $\mathrm{Br}(6 \mathrm{D})$ & $10738(2)$ & $2153(1)$ & $3732(1)$ & $66(1)$ \\
\hline $\operatorname{Br}(1 \mathrm{~W})$ & $6340(30)$ & $1457(17)$ & $3968(9)$ & $61(7)$ \\
\hline $\operatorname{Br}(2 \mathrm{~W})$ & $5182(14)$ & $2636(6)$ & $2686(6)$ & $66(4)$ \\
\hline $\operatorname{Br}(5 \mathrm{~W})$ & $2408(6)$ & $4916(3)$ & $2962(3)$ & $93(4)$ \\
\hline $\operatorname{Br}(6 \mathrm{~W})$ & 2115 ( 8 ) & $5047(3)$ & $3982(4)$ & $99(4)$ \\
\hline $\operatorname{Br}(1 \mathrm{X})$ & $7720(50)$ & $2430(30)$ & $7050(20)$ & $37(8)$ \\
\hline $\operatorname{Br}(2 \mathrm{X})$ & $8240(40)$ & $3409(19)$ & $5630(20)$ & $52(9)$ \\
\hline $\operatorname{Br}(5 X)$ & $5002(10)$ & $637(5)$ & $4853(3)$ & $67(5)$ \\
\hline $\operatorname{Br}(6 X)$ & $4442(9)$ & $-85(5)$ & $5589(5)$ & $71(5)$ \\
\hline $\operatorname{Br}(1 Y)$ & $4800(30)$ & 4775 (19) & $2215(13)$ & $60(6)$ \\
\hline $\operatorname{Br}(2 Y)$ & $3670(20)$ & $5903(9)$ & $929(7)$ & $30(4)$ \\
\hline $\operatorname{Br}(5 Y)$ & $6495(9)$ & $3135(4)$ & $-155(3)$ & 95 ( 5 ) \\
\hline $\operatorname{Br}(6 Y)$ & $7441(9)$ & $2366(4)$ & $497(6)$ & $102(6)$ \\
\hline $\mathrm{Br}(5 \mathrm{Z})$ & $10623(15)$ & $3000(7)$ & $4924(6)$ & $67(8)$ \\
\hline $\operatorname{Br}(6 \mathrm{Z})$ & 10925 (15) & $2286(6)$ & $4127(8)$ & $59(7)$ \\
\hline$C(1 A)$ & $4622(12)$ & 2761 ( 5) & $3014(4)$ & $27(9)$ \\
\hline$C(2 A)$ & $4590(11)$ & $2620(4)$ & $3430(5)$ & $65(18)$ \\
\hline$C(3 A)$ & $3996(12)$ & $2825(5)$ & $4170(4)$ & $20(8)$ \\
\hline$C(4 A)$ & 3517 (12) & $3143(5)$ & $4389(3)$ & $44(11)$ \\
\hline$C(5 A)$ & 2652 (12) & $3906(5)$ & $4318(3)$ & $68(14)$ \\
\hline$C(6 A)$ & $2388(12)$ & $4244(5)$ & $4038(4)$ & $48(13)$ \\
\hline$C(7 A)$ & $2448(13)$ & $4510(4)$ & $3255(5)$ & $47(14)$ \\
\hline$C(8 A)$ & $2763(13)$ & $4402(4)$ & $2862(4)$ & $50(11)$ \\
\hline$C(9 A)$ & $3665(12)$ & $3803(5)$ & 2449 (3) & $55(12)$ \\
\hline$C(10 A)$ & $4124(12)$ & $3398(5)$ & $2486(3)$ & $45(11)$ \\
\hline$C(11 A)$ & $4004(10)$ & 3111 ( 4) & $2843(3)$ & $41(10)$ \\
\hline$C(12 A)$ & $3938(10)$ & $2816(4)$ & $3712(4)$ & $18(8)$ \\
\hline$C(13 A)$ & $2938(10)$ & $3480(4)$ & $4170(3)$ & $40(10)$ \\
\hline
\end{tabular}




\begin{tabular}{|c|c|c|c|c|}
\hline$C(14 A)$ & $2386(10)$ & $4186(4)$ & $3585(4)$ & $54(12)$ \\
\hline$C(15 A)$ & $3045(10)$ & $3958(4)$ & $2764(3)$ & $38(12)$ \\
\hline$C(16 A)$ & $2784(8)$ & $3642(4)$ & 3049 (3) & $42(10)$ \\
\hline$C(17 A)$ & $3255(8)$ & $3226(3)$ & 3088 (3) & $38(10)$ \\
\hline$C(18 A)$ & $3223(8)$ & 3081 (3) & 3515 (3) & $15(8)$ \\
\hline$C(19 A)$ & $2731(7)$ & $3407(4)$ & 3740 (3) & $29(9)$ \\
\hline$C(20 A)$ & $2460(7)$ & $3754(4)$ & $3452(3)$ & $38(11)$ \\
\hline$C(1 B)$ & $6705(12)$ & $2318(4)$ & $6189(5)$ & $34(13)$ \\
\hline$C(2 B)$ & $6751(12)$ & $2180(4)$ & $6608(4)$ & $39(16)$ \\
\hline$C(3 B)$ & $6286(11)$ & $1545(5)$ & $7111(3)$ & $46(9)$ \\
\hline$C(4 B)$ & $5842(11)$ & $1137(4)$ & $7124(3)$ & $52(9)$ \\
\hline$C(5 B)$ & $4953(10)$ & $528(3)$ & $6659(4)$ & $34(7)$ \\
\hline$C(6 \mathrm{~B})$ & $4633(10)$ & $413(3)$ & $6247(4)$ & $39(8)$ \\
\hline$C(7 B)$ & $4548(11)$ & $671(4)$ & $5456(4)$ & $62(10)$ \\
\hline$C(8 B)$ & $4794(11)$ & $1008(4)$ & 5189 (3) & $76(12)$ \\
\hline$C(9 B)$ & $5631(12)$ & $1777(5)$ & $5166(3)$ & $54(9)$ \\
\hline$C(10 B)$ & $6103(12)$ & $2100(4)$ & $5413(4)$ & $57(10)$ \\
\hline$C(11 B)$ & $6054(10)$ & $2114(3)$ & $5867(4)$ & $36(9)$ \\
\hline$C(12 B)$ & $6149(10)$ & $1827(4)$ & $6744(3)$ & $46(10)$ \\
\hline$C(13 B)$ & $5221(9)$ & $974(3)$ & $6770(3)$ & $48(9)$ \\
\hline$C(14 B)$ & $4553(8)$ & $733(3)$ & $5909(4)$ & $54(9)$ \\
\hline$C(15 B)$ & $5068(9)$ & $1438(4)$ & $5351(3)$ & $72(11)$ \\
\hline$C(16 B)$ & $4868(7)$ & $1513(3)$ & $5768(3)$ & $54(9)$ \\
\hline$C(17 B)$ & $5352(8)$ & $1845(3)$ & $6021(3)$ & $45(9)$ \\
\hline$C(18 B)$ & $5398(8)$ & $1704(3)$ & $6452(3)$ & $44(9)$ \\
\hline$C(19 B)$ & $4943(7)$ & $1286(3)$ & $6465(3)$ & $28(7)$ \\
\hline$C(20 B)$ & $4615(6)$ & $1167(3)$ & $6042(3)$ & $41(8)$ \\
\hline$C(1 C)$ & $5338(12)$ & $4658(4)$ & $1294(5)$ & $53(14)$ \\
\hline$C(2 C)$ & $5594(12)$ & $4495(5)$ & $1696(4)$ & $55(12)$ \\
\hline$C(3 C)$ & $6431(12)$ & $3839(5)$ & 2115 (3) & $63(11)$ \\
\hline$C(4 C)$ & $6896(12)$ & $3440(5)$ & 2071 (3) & $52(10)$ \\
\hline$C(5 C)$ & $7476(12)$ & $2877(4)$ & $1519(4)$ & $56(12)$ \\
\hline$C(6 C)$ & $7509(12)$ & $2794(4)$ & $1088(5)$ & $56(10)$ \\
\hline$C(7 C)$ & $7029(12)$ & $3102(4)$ & $330(4)$ & $65(12)$ \\
\hline$C(8 C)$ & $6584(12)$ & $3450(5)$ & $108(3)$ & $64(11)$ \\
\hline$C(9 C)$ & $5708(12)$ & $4203(5)$ & $190(3)$ & $65(11)$ \\
\hline$C(10 C)$ & $5400(12)$ & 4501 (5) & $483(4)$ & 75 (13) \\
\hline$C(11 C)$ & $5763(10)$ & $4487(4)$ & $924(4)$ & $45(10)$ \\
\hline$C(12 C)$ & $6294(10)$ & $4146(4)$ & $1766(3)$ & $44(9)$ \\
\hline$C(13 C)$ & $7266(10)$ & 3311 (4) & $1674(3)$ & $40(9)$ \\
\hline$C(14 C)$ & $7335(10)$ & $3137(4)$ & $776(4)$ & $54(11)$ \\
\hline$C(15 C)$ & $6405(10)$ & $3864(4)$ & $312(3)$ & $55(10)$ \\
\hline$C(16 C)$ & $6886(8)$ & $3917(3)$ & $710(3)$ & $63(11)$ \\
\hline$C(17 C)$ & $6570(8)$ & $4223(3)$ & $1010(3)$ & $39(9)$ \\
\hline$C(18 C)$ & $6831(8)$ & $4055(3)$ & $1424(3)$ & $43(9)$ \\
\hline$C(19 C)$ & $7309(7)$ & $3645(3)$ & $1379(3)$ & $38(9)$ \\
\hline$C(20 C)$ & $7342(7)$ & $3560(3)$ & $937(3)$ & $46(9)$ \\
\hline C (1D) & $8162(8)$ & $4615(3)$ & $3654(3)$ & $38(7)$ \\
\hline$C(2 D)$ & $8243(8)$ & $4508(3)$ & $3232(3)$ & $12(5)$ \\
\hline$C(3 D)$ & $8896(8)$ & $3919(3)$ & $2725(2)$ & $40(7)$ \\
\hline$C(4 D)$ & $9377(9)$ & $3520(4)$ & $2710(2)$ & $43(8)$ \\
\hline$C(5 D)$ & $10189(9)$ & 2891 (3) & 3177 (3) & $70(11)$ \\
\hline$C(6 D)$ & 10405 (9) & $2751(3)$ & 3591 (4) & $58(9)$ \\
\hline$C(7 D)$ & $10253(9)$ & 2951 (3) & $4387(3)$ & $60(9)$ \\
\hline$C(8 D)$ & $9907(9)$ & $3264(4)$ & $4656(3)$ & $55(8)$ \\
\hline$C(9 D)$ & $9002(9)$ & $4017(4)$ & $4682(2)$ & $52(8)$ \\
\hline$C(10 D)$ & $8571(9)$ & $4351(3)$ & $4435(3)$ & $58(9)$ \\
\hline
\end{tabular}




\begin{tabular}{|c|c|c|c|c|}
\hline $\mathrm{C}(11 \mathrm{D})$ & $8743(7)$ & $4398(3)$ & $3992(3)$ & $40(8)$ \\
\hline $\mathrm{C}(12 \mathrm{D})$ & $8911(7)$ & $4175(3)$ & $3109(2)$ & $40(7)$ \\
\hline$C(13 D)$ & $9915(7)$ & $3340(3)$ & $3081(2)$ & $40(7)$ \\
\hline$C(14 D)$ & $10367(7)$ & $3047(3)$ & $3946(3)$ & $54(9)$ \\
\hline$C(15 D)$ & $9643(7)$ & 3701 (3) & $4509(2)$ & $52(9)$ \\
\hline$C(16 D)$ & 9952 (6) & $3810(3)$ & $4116(2)$ & $30(6)$ \\
\hline$C(17 D)$ & $9509(6)$ & $4152(2)$ & $3862(2)$ & $26(6)$ \\
\hline $\mathrm{C}(18 \mathrm{D})$ & $9592(6)$ & $4042(2)$ & $3428(2)$ & $32(7)$ \\
\hline$C(19 D)$ & $10085(5)$ & $3633(3)$ & $3414(2)$ & $46(8)$ \\
\hline$C(20 D)$ & $10308(5)$ & $3489(3)$ & $3839(2)$ & $46(8)$ \\
\hline $\mathrm{C}(1 \mathrm{~W})$ & $4559(18)$ & $2613(7)$ & $3536(8)$ & $11(13)$ \\
\hline$C(2 W)$ & $4468(18)$ & $2819(8)$ & $3143(7)$ & $45(17)$ \\
\hline$C(3 W)$ & $3810(20)$ & $3520(9)$ & $2740(5)$ & $35(15)$ \\
\hline$C(4 W)$ & $3340(20)$ & $3918(8)$ & $2786(6)$ & $100(30)$ \\
\hline$C(5 W)$ & $2550(20)$ & $4426(6)$ & $3330(8)$ & $100(40)$ \\
\hline$C(6 W)$ & $2350(20)$ & $4466(6)$ & $3751(9)$ & $70(20)$ \\
\hline$C(7 W)$ & $2520(20)$ & $4078(8)$ & $4492(7)$ & $60(20)$ \\
\hline$C(8 W)$ & $2870(20)$ & $3706(9)$ & $4705(6)$ & $60(20)$ \\
\hline$C(9 W)$ & $3760(20)$ & $2958(8)$ & $4619(6)$ & $45(16)$ \\
\hline $\mathrm{C}(10 \mathrm{~W})$ & 4177 (19) & $2688(7)$ & $4330(7)$ & $54(18)$ \\
\hline $\mathrm{C}(11 \mathrm{~W})$ & $3992(16)$ & $2750(6)$ & $3878(7)$ & $33(15)$ \\
\hline $\mathrm{C}(12 \mathrm{~W})$ & $3803(16)$ & $3179(7)$ & $3057(5)$ & $80(20)$ \\
\hline $\mathrm{C}(13 \mathrm{~W})$ & $2812(18)$ & $4009(7)$ & $3154(6)$ & $43(16)$ \\
\hline $\mathrm{C}(14 \mathrm{~W})$ & $2389(17)$ & $4092(7)$ & $4034(7)$ & $70(20)$ \\
\hline $\mathrm{C}(15 \mathrm{~W})$ & 3118 (17) & $3314(7)$ & $4481(5)$ & $70(20)$ \\
\hline $\mathrm{C}(16 \mathrm{~W})$ & 3225 (13) & $3028(5)$ & $3759(6)$ & $20(12)$ \\
\hline $\mathrm{C}(17 \mathrm{~W})$ & 3132 (13) & $3239(6)$ & $3355(5)$ & $46(17)$ \\
\hline $\mathrm{C}(18 \mathrm{~W})$ & $2646(13)$ & $3647(6)$ & $3403(5)$ & $70(20)$ \\
\hline $\mathrm{C}(19 \mathrm{~W})$ & $2438(12)$ & $3687(6)$ & $3836(6)$ & $29(14)$ \\
\hline $\mathrm{C}(20 \mathrm{~W})$ & $2796(12)$ & $3305(6)$ & $4055(5)$ & $90(30)$ \\
\hline$C(1 Y)$ & $5560(20)$ & $4528(9)$ & $1810(8)$ & $0(14)$ \\
\hline$C(2 Y)$ & $5440(20)$ & $4657(8)$ & $1387(9)$ & $60(40)$ \\
\hline$C(3 Y)$ & $5730(20)$ & $4422(9)$ & $612(7)$ & $29(18)$ \\
\hline$C(4 Y)$ & $6100(20)$ & $4091(10)$ & $368(6)$ & $40(20)$ \\
\hline$C(5 Y)$ & $6920(30)$ & $3316(9)$ & $398(7)$ & $60(30)$ \\
\hline$C(6 Y)$ & $7260(30)$ & $2983(8)$ & $669(8)$ & $30(20)$ \\
\hline$C(7 Y)$ & $7480(30)$ & $2739(7)$ & $1464(10)$ & $60(30)$ \\
\hline$C(8 Y)$ & $7320(30)$ & $2864(8)$ & $1876(9)$ & $50(20)$ \\
\hline $\mathrm{C}(9 \mathrm{Y})$ & $6640(30)$ & $3487(10)$ & $2336(6)$ & $40(20)$ \\
\hline C (10Y) & $6200(30)$ & $3899(10)$ & $2319(6)$ & $120(40)$ \\
\hline $\mathrm{C}(11 \mathrm{Y})$ & $6200(20)$ & 4172 ( 8 ) & $1946(6)$ & $14(15)$ \\
\hline C (12Y) & $5960(20)$ & $4442(7)$ & $1065(7)$ & $14(16)$ \\
\hline C (13Y) & $6720(20)$ & $3751(8)$ & $555(6)$ & $18(16)$ \\
\hline $\mathrm{C}(14 \mathrm{Y})$ & $7430(20)$ & $3053(7)$ & $1120(8)$ & $70(30)$ \\
\hline$C(15 Y)$ & $7110(20)$ & $3314(8)$ & $1980(7)$ & $40(20)$ \\
\hline$C(16 Y)$ & $6832(16)$ & $4040(7)$ & $1651(6)$ & $30(19)$ \\
\hline$C(17 Y)$ & $6713(16)$ & $4173(6)$ & $1218(6)$ & $0(12)$ \\
\hline C (18Y) & 7087 (15) & $3832(7)$ & $967(6)$ & $70(30)$ \\
\hline C (19Y) & $7437(15)$ & $3489(7)$ & $1245(7)$ & $24(17)$ \\
\hline C (20Y) & $7280(15)$ & $3618(7)$ & $1668(6)$ & $30(20)$ \\
\hline$C(1 X)$ & $6780(40)$ & $2170(14)$ & $6666(15)$ & $50(70)$ \\
\hline$C(2 X)$ & $6670(40)$ & $2264(13)$ & $6235(15)$ & $30(50)$ \\
\hline$C(3 X)$ & $6000(40)$ & $1963(13)$ & $5496(12)$ & $40(30)$ \\
\hline$C(4 X)$ & $5520(40)$ & $1612(14)$ & $5291(10)$ & $20(20)$ \\
\hline$C(5 X)$ & $4750(30)$ & 842 (12) & $5407(9)$ & $10(20)$ \\
\hline$C(6 X)$ & $4570(30)$ & $531(11)$ & $5712(11)$ & $30(20)$ \\
\hline$C(7 X)$ & $4760(30)$ & $355(10)$ & $6523(12)$ & $20(20)$ \\
\hline
\end{tabular}




\begin{tabular}{|c|c|c|c|c|}
\hline$C(8 X)$ & $5120(30)$ & $514(11)$ & $6916(11)$ & $10(20)$ \\
\hline$C(9 X)$ & $6010(40)$ & 1176 (15) & 7301 (9) & $80(50)$ \\
\hline$C(10 X)$ & $6420(40)$ & $1585(15)$ & $7239(11)$ & $120(70)$ \\
\hline$C(11 X)$ & $6220(40)$ & 1827 (12) & $6849(11)$ & $20(30)$ \\
\hline$C(12 x)$ & $6000(30)$ & $2022(11)$ & $5949(12)$ & $100(60)$ \\
\hline$C(13 X)$ & $5010(30)$ & $1288(11)$ & $5522(8)$ & $30(30)$ \\
\hline$C(14 X)$ & $4620(30)$ & $639(9)$ & $6158(10)$ & $30(30)$ \\
\hline$C(15 X)$ & $5370(30)$ & $972(12)$ & $6978(9)$ & $60(30)$ \\
\hline$C(16 X)$ & $5450(30)$ & $1668(9)$ & $6587(10)$ & $0(18)$ \\
\hline$C(17 X)$ & $5340(30)$ & $1764(9)$ & $6145(10)$ & $50(30)$ \\
\hline$C(18 X)$ & $4860(20)$ & $1403(9)$ & $5936(9)$ & $30(30)$ \\
\hline$C(19 X)$ & $4664(19)$ & $1084(9)$ & $6247(9)$ & $80(40)$ \\
\hline$C(20 X)$ & $5030(20)$ & $1248(10)$ & $6651(9)$ & $10(20)$ \\
\hline C (1Z) & $8030(50)$ & 4477 (19) & $3197(18)$ & $700(800)$ \\
\hline$C(2 Z)$ & $8150(50)$ & $4538(18)$ & $3632(18)$ & 47 (19) \\
\hline C ( $3 Z)$ & $8930(50)$ & $4205(18)$ & $4341(14)$ & $10(30)$ \\
\hline$C(4 Z)$ & $9480(40)$ & 3857 (18) & $4514(11)$ & $10(30)$ \\
\hline C ( 5Z) & $10360(40)$ & $3130(16)$ & $4334(11)$ & $10(30)$ \\
\hline C ( 6Z) & $10580(40)$ & $2853(14)$ & $4005(14)$ & $0(30)$ \\
\hline C ( 7 Z ) & $10340(40)$ & $2736(14)$ & $3184(14)$ & $30(40)$ \\
\hline C ( 8Z) & $9930(40)$ & $2913(15)$ & $2808(13)$ & $20(30)$ \\
\hline C (9Z) & $8900(40)$ & 3568 (19) & $2482(11)$ & $20(40)$ \\
\hline C ( $10 Z)$ & $8430(40)$ & $3950(20)$ & $2578(13)$ & $60(50)$ \\
\hline C (11Z) & $8620(40)$ & $4171(16)$ & $2982(14)$ & $50(50)$ \\
\hline C (12Z) & $8880(40)$ & $4300(15)$ & $3893(14)$ & $70(60)$ \\
\hline C (13Z) & $10020(30)$ & $3573(15)$ & $4254(10)$ & $10(30)$ \\
\hline C ( 1 4Z) & $10470(30)$ & $2995(13)$ & $3568(13)$ & $20(40)$ \\
\hline C (15Z) & $9610(40)$ & $3364(15)$ & $2781(10)$ & $0(30)$ \\
\hline C (16Z) & $9440(30)$ & $4021(12)$ & $3223(11)$ & $0(30)$ \\
\hline C (17Z) & $9560(30)$ & $4084(11)$ & $3670(12)$ & $0(30)$ \\
\hline C (18Z) & $10120(30)$ & 3727 (12) & $3848(10)$ & $30(40)$ \\
\hline C (19Z) & $10340(20)$ & $3443(12)$ & $3511(11)$ & $0(30)$ \\
\hline C (20Z) & $9920(30)$ & $3624(13)$ & $3125(10)$ & $60(50)$ \\
\hline
\end{tabular}


Table 10. Distances [A] and angles [deg] for trans-3/trans-4.

\begin{tabular}{|c|c|}
\hline $\operatorname{Pt}(1 A)-C(1 W)$ & $2.012(17)$ \\
\hline $\operatorname{Pt}(1 \mathrm{~A})-\mathrm{C}(2 \mathrm{~A})$ & $2.089(10)$ \\
\hline $\operatorname{Pt}(1 \mathrm{~A})-\mathrm{P}(1 \mathrm{~A})$ & $2.301(6)$ \\
\hline$P t(1 A)-P(2 A)$ & $2.301(6)$ \\
\hline $\operatorname{Pt}(1 \mathrm{~A})-\operatorname{Br}(1 \mathrm{~W})$ & $2.45(5)$ \\
\hline $\operatorname{Pt}(1 A)-B r(2 A)$ & $2.56(3)$ \\
\hline Pt (1B) $-C(1 B)$ & $2.018(9)$ \\
\hline Pt (1B) $-C(2 X)$ & $2.22(3)$ \\
\hline Pt (1B) -P (1B) & $2.285(7)$ \\
\hline Pt (1B) $-P(2 B)$ & $2.307(7)$ \\
\hline Pt (1B) $-B r(2 X)$ & $2.37(7)$ \\
\hline Pt (1B) $-\mathrm{Br}(1 \mathrm{~B})$ & $2.547(16)$ \\
\hline $\operatorname{Pt}(1 C)-C(1 C)$ & $2.035(10)$ \\
\hline Pt (1C) $-C(2 Y)$ & $2.17(2)$ \\
\hline Pt (1C) $-P(1 C)$ & $2.298(7)$ \\
\hline $\operatorname{Pt}(1 \mathrm{C})-\mathrm{P}(2 \mathrm{C})$ & $2.304(8)$ \\
\hline $\operatorname{Pt}(1 \mathrm{C})-\mathrm{Br}(2 \mathrm{Y})$ & $2.51(3)$ \\
\hline Pt (1C) $-B r(1 C)$ & $2.521(12)$ \\
\hline Pt (1D) $-C(1 D)$ & $2.059(7)$ \\
\hline Pt (1D) $-C(2 Z)$ & $2.26(4)$ \\
\hline$P t(1 D)-P(2 D)$ & $2.306(8)$ \\
\hline Pt (1D) -P (1D) & $2.306(7)$ \\
\hline Pt (1D) $-\operatorname{Br}(1 D)$ & $2.503(3)$ \\
\hline$P(1 A)-C(21 A)$ & $1.80(2)$ \\
\hline$P(1 A)-C(25 A)$ & $1.81(3)$ \\
\hline$P(1 A)-C(23 A)$ & $1.82(3)$ \\
\hline$P(2 A)-C(27 A)$ & $1.77(2)$ \\
\hline$P(2 A)-C(31 A)$ & $1.85(2)$ \\
\hline$P(2 A)-C(29 A)$ & $1.86(2)$ \\
\hline$P(1 B)-C(21 B)$ & $1.85(2)$ \\
\hline$P(1 B)-C(23 B)$ & $1.85(2)$ \\
\hline$P(1 B)-C(25 B)$ & $1.87(2)$ \\
\hline$P(2 B)-C(29 B)$ & $1.76(3)$ \\
\hline$P(2 B)-C(31 B)$ & $1.86(3)$ \\
\hline$P(2 B)-C(27 B)$ & $1.98(3)$ \\
\hline$P(1 C)-C(21 C)$ & $1.74(3)$ \\
\hline$P(1 C)-C(23 C)$ & $1.77(3)$ \\
\hline$P(1 C)-C(25 C)$ & $1.94(5)$ \\
\hline$P(2 C)-C(31 C)$ & $1.73(4)$ \\
\hline$P(2 C)-C(29 C)$ & $1.83(2)$ \\
\hline$P(2 C)-C(27 C)$ & $1.83(3)$ \\
\hline$P(1 D)-C(25 D)$ & $1.73(3)$ \\
\hline$P(1 D)-C(23 D)$ & $1.81(3)$ \\
\hline$P(1 D)-C(21 D)$ & $1.87(5)$ \\
\hline$P(2 D)-C(31 D)$ & $1.79(3)$ \\
\hline$P(2 D)-C(29 D)$ & $1.86(3)$ \\
\hline$P(2 D)-C(27 D)$ & $1.87(3)$ \\
\hline$C(21 A)-C(22 A)$ & $1.56(3)$ \\
\hline$C(23 A)-C(24 A)$ & $1.49(3)$ \\
\hline$C(25 A)-C(26 A)$ & $1.51(3)$ \\
\hline$C(27 A)-C(28 A)$ & $1.57(3)$ \\
\hline$C(29 A)-C(30 A)$ & $1.56(3)$ \\
\hline$C(31 A)-C(32 A)$ & $1.53(3)$ \\
\hline
\end{tabular}




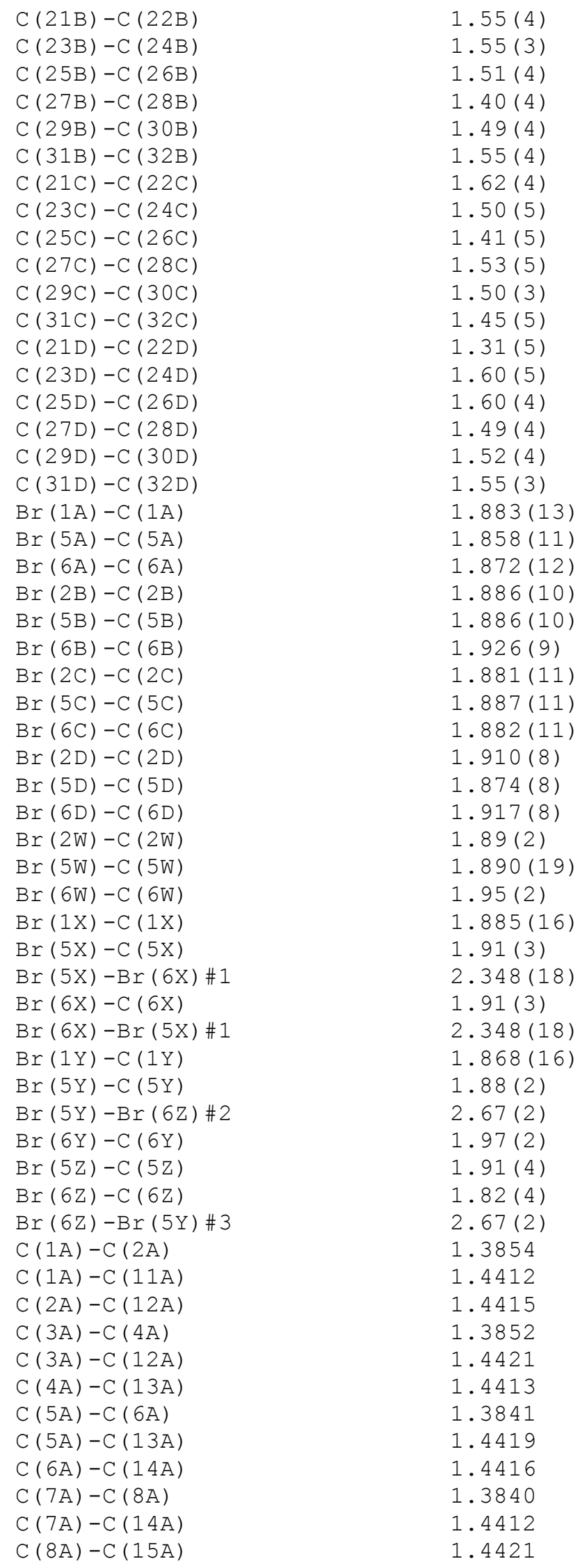

$1.55(3)$

$1.51(4)$

$1.40(4)$

$1.49(4)$

$1.55(4)$

$1.62(4)$

$1.50(5)$

$1.41(5)$

$1.53(5)$

$1.50(3)$

$1.45(5)$

$1.31(5)$

$1.60(5)$

$1.60(4)$

$1.49(4)$

$1.52(4)$

$1.55(3)$

$1.883(13)$

$1.858(11)$

$1.872(12)$

$1.886(10)$

$1.886(10)$

$1.926(9)$

1.881 (11)

1.887 (11)

$1.882(11)$

$1.910(8)$

$1.874(8)$

$1.917(8)$

$1.89(2)$

1.890 (19)

$1.95(2)$

$1.885(16)$

1.91 (3)

2. 348 (18)

$1.91(3)$

2. $348(18)$

$1.868(16)$

$1.88(2)$

$2.67(2)$

$1.97(2)$

$1.91(4)$

$1.82(4)$

2.67 (2)

1.3854

1. 4412

1.4415

1. 3852

1. 4421

1.4413

1.3841

1.4419

1. 4416

1. 3840

1. 4412

1. 4421 


\begin{tabular}{|c|c|}
\hline$C(9 A)-C(10 A)$ & 1.3851 \\
\hline$C(9 A)-C(15 A)$ & 1.4415 \\
\hline$C(10 A)-C(11 A)$ & 1.4414 \\
\hline$C(11 A)-C(17 A)$ & 1.3824 \\
\hline$C(12 A)-C(18 A)$ & 1.3821 \\
\hline$C(13 A)-C(19 A)$ & 1.3829 \\
\hline$C(14 A)-C(20 A)$ & 1.3826 \\
\hline$C(15 A)-C(16 A)$ & 1.3831 \\
\hline$C(16 A)-C(17 A)$ & 1.4201 \\
\hline$C(16 A)-C(20 A)$ & 1.4218 \\
\hline$C(17 A)-C(18 A)$ & 1.4222 \\
\hline$C(18 A)-C(19 A)$ & 1.4218 \\
\hline$C(19 A)-C(20 A)$ & 1.4218 \\
\hline$C(1 B)-C(2 B)$ & 1.3852 \\
\hline$C(1 B)-C(11 B)$ & 1.4428 \\
\hline$C(2 B)-C(12 B)$ & 1.4410 \\
\hline$C(3 B)-C(4 B)$ & 1.3842 \\
\hline$C(3 B)-C(12 B)$ & 1.4419 \\
\hline$C(4 B)-C(13 B)$ & 1.4420 \\
\hline$C(5 B)-C(6 B)$ & 1.3861 \\
\hline$C(5 B)-C(13 B)$ & 1.4409 \\
\hline$C(6 B)-C(14 B)$ & 1.4408 \\
\hline$C(7 B)-C(8 B)$ & 1.3861 \\
\hline$C(7 B)-C(14 B)$ & 1.4429 \\
\hline$C(8 B)-C(15 B)$ & 1.4407 \\
\hline$C(9 B)-C(10 B)$ & 1.3843 \\
\hline$C(9 B)-C(15 B)$ & 1.4417 \\
\hline$C(10 B)-C(11 B)$ & 1.4405 \\
\hline$C(11 B)-C(17 B)$ & 1.3827 \\
\hline$C(12 B)-C(18 B)$ & 1.3828 \\
\hline$C(13 B)-C(19 B)$ & 1.3828 \\
\hline$C(14 B)-C(20 B)$ & 1.3827 \\
\hline$C(15 B)-C(16 B)$ & 1.3818 \\
\hline$C(16 B)-C(20 B)$ & 1.4204 \\
\hline$C(16 B)-C(17 B)$ & 1.4218 \\
\hline$C(17 B)-C(18 B)$ & 1.4224 \\
\hline$C(18 B)-C(19 B)$ & 1.4198 \\
\hline$C(19 B)-C(20 B)$ & 1.4219 \\
\hline$C(1 C)-C(2 C)$ & 1.3834 \\
\hline$C(1 C)-C(11 C)$ & 1.4418 \\
\hline$C(2 C)-C(12 C)$ & 1.4415 \\
\hline$C(3 C)-C(4 C)$ & 1.3835 \\
\hline$C(3 C)-C(12 C)$ & 1.4418 \\
\hline$C(4 C)-C(13 C)$ & 1.4428 \\
\hline$C(5 C)-C(6 C)$ & 1.3861 \\
\hline$C(5 C)-C(13 C)$ & 1.4411 \\
\hline$C(6 C)-C(14 C)$ & 1.4407 \\
\hline$C(7 C)-C(8 C)$ & 1.3845 \\
\hline$C(7 C)-C(14 C)$ & 1.4421 \\
\hline$C(8 C)-C(15 C)$ & 1.4424 \\
\hline$C(9 C)-C(10 C)$ & 1.3841 \\
\hline$C(9 C)-C(15 C)$ & 1.4422 \\
\hline$C(10 C)-C(11 C)$ & 1.4416 \\
\hline$C(11 C)-C(17 C)$ & 1.3823 \\
\hline$C(12 C)-C(18 C)$ & 1.3834 \\
\hline
\end{tabular}




\begin{tabular}{|c|c|}
\hline$C(13 C)-C(19 C)$ & 1.3808 \\
\hline$C(14 C)-C(20 C)$ & 1.3825 \\
\hline$C(15 C)-C(16 C)$ & 1.3824 \\
\hline$C(16 C)-C(20 C)$ & 1.4206 \\
\hline$C(16 C)-C(17 C)$ & 1.4209 \\
\hline$C(17 C)-C(18 C)$ & 1.4221 \\
\hline$C(18 C)-C(19 C)$ & 1.4216 \\
\hline$C(19 C)-C(20 C)$ & 1.4224 \\
\hline$C(1 D)-C(2 D)$ & 1.3835 \\
\hline$C(1 D)-C(11 D)$ & 1.4417 \\
\hline$C(2 D)-C(12 D)$ & 1.4420 \\
\hline$C(3 D)-C(4 D)$ & 1.3842 \\
\hline$C(3 D)-C(12 D)$ & 1.4395 \\
\hline$C(4 D)-C(13 D)$ & 1.4422 \\
\hline$C(5 D)-C(6 D)$ & 1.3833 \\
\hline$C(5 D)-C(13 D)$ & 1.4411 \\
\hline$C(6 D)-C(14 D)$ & 1.4424 \\
\hline$C(7 D)-C(8 D)$ & 1.3840 \\
\hline$C(7 D)-C(14 D)$ & 1.4425 \\
\hline$C(8 D)-C(15 D)$ & 1.4424 \\
\hline$C(9 D)-C(10 D)$ & 1.3847 \\
\hline$C(9 D)-C(15 D)$ & 1.4421 \\
\hline$C(10 D)-C(11 D)$ & 1.4427 \\
\hline$C(11 D)-C(17 D)$ & 1.3820 \\
\hline$C(12 D)-C(18 D)$ & 1.3822 \\
\hline$C(13 D)-C(19 D)$ & 1.3826 \\
\hline$C(14 D)-C(20 D)$ & 1.3828 \\
\hline$C(15 D)-C(16 D)$ & 1.3822 \\
\hline$C(16 D)-C(20 D)$ & 1.4209 \\
\hline$C(16 D)-C(17 D)$ & 1.4214 \\
\hline$C(17 D)-C(18 D)$ & 1.423 \\
\hline$C(18 D)-C(19 D)$ & 1.4202 \\
\hline$C(19 D)-C(20 D)$ & 1.4213 \\
\hline$C(1 W)-C(2 W)$ & 1.3840 \\
\hline$C(1 W)-C(11 W)$ & 1.4416 \\
\hline$C(2 W)-C(12 W)$ & 1.4410 \\
\hline$C(3 W)-C(4 W)$ & 1.3855 \\
\hline$C(3 W)-C(12 W)$ & 1.4425 \\
\hline$C(4 W)-C(13 W)$ & 1.4420 \\
\hline$C(5 W)-C(6 W)$ & 1.3841 \\
\hline$C(5 W)-C(13 W)$ & 1.4424 \\
\hline$C(6 \mathrm{~W})-\mathrm{C}(14 \mathrm{~W})$ & 1.442 \\
\hline$C(7 W)-C(8 W)$ & 1.383 \\
\hline$C(7 W)-C(14 W)$ & 1.441 \\
\hline$C(8 W)-C(15 W)$ & 1.440 \\
\hline$C(9 W)-C(10 W)$ & 1.3842 \\
\hline$C(9 W)-C(15 W)$ & 1.4425 \\
\hline$C(10 W)-C(11 W)$ & 1.440 \\
\hline$C(11 W)-C(16 W)$ & 1.382 \\
\hline$C(12 W)-C(17 W)$ & 1.381 \\
\hline$C(13 W)-C(18 W)$ & 1.381 \\
\hline$C(14 W)-C(19 W)$ & 1.3831 \\
\hline$C(15 W)-C(20 W)$ & 1.383 \\
\hline$C(16 W)-C(20 W)$ & 1.421 \\
\hline$C(16 \mathrm{~W})-\mathrm{C}(17 \mathrm{~W})$ & 1.421 \\
\hline$C(17 W)-C(18 W)$ & 1.422 \\
\hline$C(18 W)-C(19 W)$ & 1.421 \\
\hline
\end{tabular}




\begin{tabular}{|c|c|}
\hline$C(19 W)-C(20 W)$ & 1.4206 \\
\hline$C(1 Y)-C(2 Y)$ & 1.3858 \\
\hline$C(1 Y)-C(11 Y)$ & 1.4418 \\
\hline$C(2 Y)-C(12 Y)$ & 1.4420 \\
\hline$C(3 Y)-C(4 Y)$ & 1.3851 \\
\hline$C(3 Y)-C(12 Y)$ & 1.4424 \\
\hline$C(4 Y)-C(13 Y)$ & 1.4409 \\
\hline$C(5 Y)-C(6 Y)$ & 1.3839 \\
\hline$C(5 Y)-C(13 Y)$ & 1.4430 \\
\hline$C(6 Y)-C(14 Y)$ & 1.4417 \\
\hline$C(7 Y)-C(8 Y)$ & 1.3855 \\
\hline$C(7 Y)-C(14 Y)$ & 1.4419 \\
\hline$C(8 Y)-C(15 Y)$ & 1.4415 \\
\hline$C(9 Y)-C(10 Y)$ & 1.3865 \\
\hline$C(9 Y)-C(15 Y)$ & 1.4422 \\
\hline$C(10 Y)-C(11 Y)$ & 1.4405 \\
\hline$C(11 Y)-C(16 Y)$ & 1.3819 \\
\hline$C(12 Y)-C(17 Y)$ & 1.3811 \\
\hline$C(13 Y)-C(18 Y)$ & 1.3823 \\
\hline$C(14 Y)-C(19 Y)$ & 1.3814 \\
\hline$C(15 Y)-C(20 Y)$ & 1.3828 \\
\hline$C(16 Y)-C(20 Y)$ & 1.4218 \\
\hline$C(16 Y)-C(17 Y)$ & 1.4219 \\
\hline$C(17 Y)-C(18 Y)$ & 1.4219 \\
\hline$C(18 Y)-C(19 Y)$ & 1.4224 \\
\hline$C(19 Y)-C(20 Y)$ & 1.4215 \\
\hline$C(1 X)-C(2 X)$ & 1.3846 \\
\hline$C(1 X)-C(11 X)$ & 1.4416 \\
\hline$C(2 X)-C(12 X)$ & 1.4428 \\
\hline$C(3 X)-C(4 X)$ & 1.3836 \\
\hline$C(3 X)-C(12 X)$ & 1.4416 \\
\hline$C(4 X)-C(13 X)$ & 1.4418 \\
\hline$C(5 X)-C(6 X)$ & 1.3858 \\
\hline$C(5 X)-C(13 X)$ & 1.4415 \\
\hline$C(6 X)-C(14 X)$ & 1.4415 \\
\hline$C(7 X)-C(8 X)$ & 1.3855 \\
\hline$C(7 X)-C(14 X)$ & 1.4410 \\
\hline$C(8 X)-C(15 X)$ & 1.4397 \\
\hline$C(9 X)-C(10 X)$ & 1.3846 \\
\hline$C(9 X)-C(15 X)$ & 1.4421 \\
\hline$C(10 X)-C(11 X)$ & 1.4414 \\
\hline$C(11 X)-C(16 X)$ & 1.3824 \\
\hline$C(12 X)-C(17 X)$ & 1.3826 \\
\hline$C(13 X)-C(18 X)$ & 1.3837 \\
\hline$C(14 X)-C(19 X)$ & 1.3824 \\
\hline$C(15 X)-C(20 X)$ & 1.3824 \\
\hline$C(16 X)-C(17 X)$ & 1.4211 \\
\hline$C(16 X)-C(20 X)$ & 1.4214 \\
\hline$C(17 X)-C(18 X)$ & 1.4209 \\
\hline$C(18 X)-C(19 X)$ & 1.4196 \\
\hline$C(19 X)-C(20 X)$ & 1.4228 \\
\hline$C(1 Z)-C(2 Z)$ & 1.3823 \\
\hline$C(1 Z)-C(11 Z)$ & 1.4423 \\
\hline$C(2 Z)-C(12 Z)$ & 1.4426 \\
\hline C ( $3 Z)-C(4 Z)$ & 1.3847 \\
\hline$C(3 Z)-C(12 Z)$ & 1.4409 \\
\hline$C(4 Z)-C(13 Z)$ & 1.4407 \\
\hline
\end{tabular}




\begin{tabular}{|c|c|}
\hline$C(5 Z)-C(6 Z)$ & 1.3853 \\
\hline$C(5 Z)-C(13 Z)$ & 1.4413 \\
\hline$C(6 Z)-C(14 Z)$ & 1.4420 \\
\hline$C(7 Z)-C(8 Z)$ & 1.3845 \\
\hline$C(7 Z)-C(14 Z)$ & 1.4421 \\
\hline$C(8 Z)-C(15 Z)$ & 1.4417 \\
\hline$C(9 Z)-C(10 Z)$ & 1.3840 \\
\hline C (9Z) - C (15Z) & 1.4410 \\
\hline$C(10 Z)-C(11 Z)$ & 1.4419 \\
\hline$C(11 Z)-C(16 Z)$ & 1.3834 \\
\hline$C(12 Z)-C(17 Z)$ & 1.3828 \\
\hline$C(13 Z)-C(18 Z)$ & 1.3835 \\
\hline C (14Z) -C (19Z) & 1.3815 \\
\hline$C(15 Z)-C(20 Z)$ & 1.3830 \\
\hline$C(16 Z)-C(20 Z)$ & 1.4215 \\
\hline$C(16 Z)-C(17 Z)$ & 1.4218 \\
\hline$C(17 Z)-C(18 Z)$ & 1.4207 \\
\hline$C(18 Z)-C(19 Z)$ & 1.4210 \\
\hline$C(19 Z)-C(20 Z)$ & 1.4205 \\
\hline$C(1 W)-P t(1 A)-C(2 A)$ & $9.3(8)$ \\
\hline$C(1 W)-P t(1 A)-P(1 A)$ & $95.3(8)$ \\
\hline$C(2 A)-P t(1 A)-P(1 A)$ & $94.9(5)$ \\
\hline$C(1 W)-P t(1 A)-P(2 A)$ & $90.3(8)$ \\
\hline$C(2 A)-P t(1 A)-P(2 A)$ & $89.5(5)$ \\
\hline$P(1 A)-P t(1 A)-P(2 A)$ & $171.6(2)$ \\
\hline$C(1 W)-\operatorname{Pt}(1 A)-B r(1 W)$ & $169.5(10)$ \\
\hline$C(2 A)-\operatorname{Pt}(1 A)-B r(1 W)$ & $177.0(12)$ \\
\hline$P(1 A)-\operatorname{Pt}(1 A)-B r(1 W)$ & $88.0(11)$ \\
\hline$P(2 A)-P t(1 A)-B r(1 W)$ & $87.6(11)$ \\
\hline$C(1 W)-P t(1 A)-B r(2 A)$ & $162.3(8)$ \\
\hline$C(2 A)-\operatorname{Pt}(1 A)-B r(2 A)$ & $171.2(6)$ \\
\hline$P(1 A)-P t(1 A)-B r(2 A)$ & $89.0(6)$ \\
\hline$P(2 A)-P t(1 A)-B r(2 A)$ & $87.6(6)$ \\
\hline $\operatorname{Br}(1 W)-\operatorname{Pt}(1 A)-\operatorname{Br}(2 A)$ & $7.3(11)$ \\
\hline$C(1 B)-P t(1 B)-C(2 X)$ & $2.6(15)$ \\
\hline$C(1 B)-P t(1 B)-P(1 B)$ & $93.0(6)$ \\
\hline$C(2 X)-P t(1 B)-P(1 B)$ & $92.0(17)$ \\
\hline$C(1 B)-P t(1 B)-P(2 B)$ & $88.4(6)$ \\
\hline$C(2 X)-P t(1 B)-P(2 B)$ & $89.1(17)$ \\
\hline$P(1 B)-P t(1 B)-P(2 B)$ & $175.7(3)$ \\
\hline$C(1 B)-\operatorname{Pt}(1 B)-B r(2 X)$ & $168.7(17)$ \\
\hline$C(2 X)-\operatorname{Pt}(1 B)-B r(2 X)$ & $171(2)$ \\
\hline$P(1 B)-P t(1 B)-B r(2 X)$ & $87.1(14)$ \\
\hline$P(2 B)-P t(1 B)-B r(2 X)$ & $92.4(14)$ \\
\hline$C(1 B)-P t(1 B)-B r(1 B)$ & $174.9(6)$ \\
\hline$C(2 X)-P t(1 B)-B r(1 B)$ & $177.5(12)$ \\
\hline$P(1 B)-P t(1 B)-B r(1 B)$ & $88.7(4)$ \\
\hline$P(2 B)-P t(1 B)-B r(1 B)$ & $90.3(4)$ \\
\hline $\operatorname{Br}(2 X)-\operatorname{Pt}(1 B)-B r(1 B)$ & $7(2)$ \\
\hline$C(1 C)-P t(1 C)-C(2 Y)$ & $7.9(10)$ \\
\hline$C(1 C)-P t(1 C)-P(1 C)$ & $91.2(6)$ \\
\hline$C(2 Y)-P t(1 C)-P(1 C)$ & $94.9(10)$ \\
\hline$C(1 C)-P t(1 C)-P(2 C)$ & $92.4(6)$ \\
\hline$C(2 Y)-P t(1 C)-P(2 C)$ & $87.9(10)$ \\
\hline$P(1 C)-P t(1 C)-P(2 C)$ & $172.6(3)$ \\
\hline$C(1 C)-\operatorname{Pt}(1 C)-B r(2 Y)$ & $169.8(7)$ \\
\hline
\end{tabular}




\begin{tabular}{|c|c|}
\hline $\mathrm{C}(2 \mathrm{Y})-\operatorname{Pt}(1 \mathrm{C})-\mathrm{Br}(2 \mathrm{Y})$ & $175.8(11)$ \\
\hline $\mathrm{P}(1 \mathrm{C})-\mathrm{Pt}(1 \mathrm{C})-\mathrm{Br}(2 \mathrm{Y})$ & $87.9(6)$ \\
\hline $\mathrm{P}(2 \mathrm{C})-\mathrm{Pt}(1 \mathrm{C})-\mathrm{Br}(2 \mathrm{Y})$ & $89.8(6)$ \\
\hline$C(1 C)-\operatorname{Pt}(1 C)-\operatorname{Br}(1 C)$ & $174.5(6)$ \\
\hline$C(2 Y)-\operatorname{Pt}(1 C)-B r(1 C)$ & $172.0(10)$ \\
\hline$P(1 C)-P t(1 C)-B r(1 C)$ & $92.5(3)$ \\
\hline $\mathrm{P}(2 \mathrm{C})-\mathrm{Pt}(1 \mathrm{C})-\mathrm{Br}(1 \mathrm{C})$ & $84.4(3)$ \\
\hline $\mathrm{Br}(2 \mathrm{Y})-\operatorname{Pt}(1 \mathrm{C})-\mathrm{Br}(1 \mathrm{C})$ & $7.8(7)$ \\
\hline$C(1 D)-P t(1 D)-C(2 Z)$ & $4(2)$ \\
\hline$C(1 D)-P t(1 D)-P(2 D)$ & $90.8(4)$ \\
\hline$C(2 Z)-P t(1 D)-P(2 D)$ & $94(2)$ \\
\hline$C(1 D)-P t(1 D)-P(1 D)$ & $92.7(4)$ \\
\hline$C(2 Z)-P t(1 D)-P(1 D)$ & $89(2)$ \\
\hline$P(2 D)-P t(1 D)-P(1 D)$ & $176.3(3)$ \\
\hline$C(1 D)-\operatorname{Pt}(1 D)-B r(1 D)$ & $175.9(3)$ \\
\hline$C(2 Z)-\operatorname{Pt}(1 D)-B r(1 D)$ & $177.1(15)$ \\
\hline$P(2 D)-\operatorname{Pt}(1 D)-B r(1 D)$ & $86.9(2)$ \\
\hline$P(1 D)-P t(1 D)-B r(1 D)$ & $89.7(2)$ \\
\hline$C(21 A)-P(1 A)-C(25 A)$ & $105.1(12)$ \\
\hline$C(21 A)-P(1 A)-C(23 A)$ & $105.3(12)$ \\
\hline$C(25 A)-P(1 A)-C(23 A)$ & $102.6(13)$ \\
\hline$C(21 A)-P(1 A)-P t(1 A)$ & $115.5(7)$ \\
\hline$C(25 A)-P(1 A)-P t(1 A)$ & $110.4(8)$ \\
\hline$C(23 A)-P(1 A)-P t(1 A)$ & $116.5(11)$ \\
\hline$C(27 A)-P(2 A)-C(31 A)$ & $103.4(13)$ \\
\hline$C(27 A)-P(2 A)-C(29 A)$ & $107.1(12)$ \\
\hline$C(31 A)-P(2 A)-C(29 A)$ & $104.5(12)$ \\
\hline$C(27 A)-P(2 A)-P t(1 A)$ & $113.7(10)$ \\
\hline$C(31 A)-P(2 A)-P t(1 A)$ & $111.6(10)$ \\
\hline$C(29 A)-P(2 A)-P t(1 A)$ & $115.6(8)$ \\
\hline$C(21 B)-P(1 B)-C(23 B)$ & $101.9(11)$ \\
\hline$C(21 B)-P(1 B)-C(25 B)$ & $103.0(14)$ \\
\hline$C(23 B)-P(1 B)-C(25 B)$ & $107.9(12)$ \\
\hline$C(21 B)-P(1 B)-P t(1 B)$ & $110.5(9)$ \\
\hline$C(23 B)-P(1 B)-P t(1 B)$ & $116.4(7)$ \\
\hline$C(25 B)-P(1 B)-P t(1 B)$ & $115.5(9)$ \\
\hline$C(29 B)-P(2 B)-C(31 B)$ & $106.6(15)$ \\
\hline$C(29 B)-P(2 B)-C(27 B)$ & $110.2(16)$ \\
\hline$C(31 B)-P(2 B)-C(27 B)$ & $102.1(16)$ \\
\hline$C(29 B)-P(2 B)-P t(1 B)$ & $112.8(13)$ \\
\hline$C(31 B)-P(2 B)-P t(1 B)$ & $113.7(10)$ \\
\hline$C(27 B)-P(2 B)-P t(1 B)$ & $110.8(10)$ \\
\hline$C(21 C)-P(1 C)-C(23 C)$ & $109(2)$ \\
\hline$C(21 C)-P(1 C)-C(25 C)$ & $98(2)$ \\
\hline$C(23 C)-P(1 C)-C(25 C)$ & $99(2)$ \\
\hline$C(21 C)-P(1 C)-P t(1 C)$ & $117.6(10)$ \\
\hline$C(23 C)-P(1 C)-P t(1 C)$ & $118.7(12)$ \\
\hline$C(25 C)-P(1 C)-P t(1 C)$ & $111.0(15)$ \\
\hline$C(31 C)-P(2 C)-C(29 C)$ & $110.6(16)$ \\
\hline$C(31 C)-P(2 C)-C(27 C)$ & $101(2)$ \\
\hline$C(29 C)-P(2 C)-C(27 C)$ & $101.9(13)$ \\
\hline$C(31 C)-P(2 C)-P t(1 C)$ & $112.2(13)$ \\
\hline$C(29 C)-P(2 C)-P t(1 C)$ & $116.2(9)$ \\
\hline$C(27 C)-P(2 C)-P t(1 C)$ & $113.3(12)$ \\
\hline$C(25 D)-P(1 D)-C(23 D)$ & $107.8(14)$ \\
\hline$C(25 D)-P(1 D)-C(21 D)$ & $94(2)$ \\
\hline$C(23 D)-P(1 D)-C(21 D)$ & $104(3)$ \\
\hline
\end{tabular}




\begin{tabular}{|c|c|}
\hline$C(25 D)-P(1 D)-P t(1 D)$ & $116.5(10)$ \\
\hline$C(23 D)-P(1 D)-P t(1 D)$ & $114.8(11)$ \\
\hline$C(21 D)-P(1 D)-P t(1 D)$ & $116.7(16)$ \\
\hline$C(31 D)-P(2 D)-C(29 D)$ & $103.5(14)$ \\
\hline$C(31 D)-P(2 D)-C(27 D)$ & $108.3(14)$ \\
\hline$C(29 D)-P(2 D)-C(27 D)$ & $101.3(14)$ \\
\hline$C(31 D)-P(2 D)-P t(1 D)$ & $117.5(9)$ \\
\hline$C(29 D)-P(2 D)-P t(1 D)$ & $114.1(10)$ \\
\hline$C(27 D)-P(2 D)-P t(1 D)$ & $110.6(11)$ \\
\hline$C(22 A)-C(21 A)-P(1 A)$ & $113.6(15)$ \\
\hline$C(24 A)-C(23 A)-P(1 A)$ & $117.8(19)$ \\
\hline$C(26 A)-C(25 A)-P(1 A)$ & $113.2(18)$ \\
\hline$C(28 A)-C(27 A)-P(2 A)$ & $117.5(19)$ \\
\hline$C(30 A)-C(29 A)-P(2 A)$ & $114.6(16)$ \\
\hline$C(32 A)-C(31 A)-P(2 A)$ & $117(2)$ \\
\hline$C(22 B)-C(21 B)-P(1 B)$ & $114(2)$ \\
\hline$C(24 B)-C(23 B)-P(1 B)$ & $115.5(15)$ \\
\hline$C(26 B)-C(25 B)-P(1 B)$ & $117(2)$ \\
\hline$C(28 B)-C(27 B)-P(2 B)$ & $105(3)$ \\
\hline$C(30 B)-C(29 B)-P(2 B)$ & $112(2)$ \\
\hline$C(32 B)-C(31 B)-P(2 B)$ & $113(2)$ \\
\hline$C(22 C)-C(21 C)-P(1 C)$ & $113(2)$ \\
\hline$C(24 C)-C(23 C)-P(1 C)$ & $115(3)$ \\
\hline$C(26 C)-C(25 C)-P(1 C)$ & $115(3)$ \\
\hline$C(28 C)-C(27 C)-P(2 C)$ & $115(3)$ \\
\hline$C(30 C)-C(29 C)-P(2 C)$ & $116.2(19)$ \\
\hline$C(32 C)-C(31 C)-P(2 C)$ & $118(3)$ \\
\hline$C(22 D)-C(21 D)-P(1 D)$ & $108(4)$ \\
\hline$C(24 D)-C(23 D)-P(1 D)$ & $110(2)$ \\
\hline$C(26 D)-C(25 D)-P(1 D)$ & $116(2)$ \\
\hline$C(28 D)-C(27 D)-P(2 D)$ & $111(2)$ \\
\hline$C(30 D)-C(29 D)-P(2 D)$ & $110.4(19)$ \\
\hline$C(32 D)-C(31 D)-P(2 D)$ & $117(2)$ \\
\hline$C(5 X)-B r(5 X)-B r(6 X) \# 1$ & $149.9(15)$ \\
\hline$C(6 X)-B r(6 X)-B r(5 X) \# 1$ & $142.2(15)$ \\
\hline $\mathrm{C}(5 Y)-\operatorname{Br}(5 Y)-\operatorname{Br}(6 Z) \# 2$ & $168.3(12)$ \\
\hline $\mathrm{C}(6 \mathrm{Z})-\mathrm{Br}(6 \mathrm{Z})-\mathrm{Br}(5 \mathrm{Y}) \# 3$ & $133.6(19)$ \\
\hline$C(2 A)-C(1 A)-C(11 A)$ & 121.5 \\
\hline$C(2 A)-C(1 A)-B r(1 A)$ & $122.0(7)$ \\
\hline$C(11 A)-C(1 A)-B r(1 A)$ & $116.5(7)$ \\
\hline$C(1 A)-C(2 A)-C(12 A)$ & 121.6 \\
\hline$C(1 A)-C(2 A)-P t(1 A)$ & $122.2(6)$ \\
\hline$C(12 A)-C(2 A)-P t(1 A)$ & $116.2(6)$ \\
\hline$C(4 A)-C(3 A)-C(12 A)$ & 121.5 \\
\hline$C(3 A)-C(4 A)-C(13 A)$ & 121.6 \\
\hline$C(6 A)-C(5 A)-C(13 A)$ & 121.5 \\
\hline$C(6 A)-C(5 A)-B r(5 A)$ & $120.7(7)$ \\
\hline$C(13 A)-C(5 A)-B r(5 A)$ & $117.5(7)$ \\
\hline$C(5 A)-C(6 A)-C(14 A)$ & 121.5 \\
\hline$C(5 A)-C(6 A)-B r(6 A)$ & $122.5(7)$ \\
\hline$C(14 A)-C(6 A)-B r(6 A)$ & $116.0(7)$ \\
\hline$C(8 A)-C(7 A)-C(14 A)$ & 121.6 \\
\hline$C(7 A)-C(8 A)-C(15 A)$ & 121.6 \\
\hline$C(10 A)-C(9 A)-C(15 A)$ & 121.5 \\
\hline$C(9 A)-C(10 A)-C(11 A)$ & 121.6 \\
\hline
\end{tabular}




\begin{tabular}{|c|c|}
\hline$C(17 A)-C(11 A)-C(1 A)$ & 115.0 \\
\hline$C(17 A)-C(11 A)-C(10 A)$ & 114.8 \\
\hline$C(1 A)-C(11 A)-C(10 A)$ & 129.6 \\
\hline$C(18 A)-C(12 A)-C(2 A)$ & 114.9 \\
\hline$C(18 A)-C(12 A)-C(3 A)$ & 114.9 \\
\hline$C(2 A)-C(12 A)-C(3 A)$ & 129.6 \\
\hline$C(19 A)-C(13 A)-C(4 A)$ & 114.9 \\
\hline$C(19 A)-C(13 A)-C(5 A)$ & 115.0 \\
\hline$C(4 A)-C(13 A)-C(5 A)$ & 129.6 \\
\hline$C(20 A)-C(14 A)-C(7 A)$ & 114.9 \\
\hline$C(20 A)-C(14 A)-C(6 A)$ & 114.9 \\
\hline$C(7 A)-C(14 A)-C(6 A)$ & 129.6 \\
\hline$C(16 A)-C(15 A)-C(9 A)$ & 114.9 \\
\hline$C(16 A)-C(15 A)-C(8 A)$ & 114.9 \\
\hline$C(9 A)-C(15 A)-C(8 A)$ & 129.7 \\
\hline$C(15 A)-C(16 A)-C(17 A)$ & 122.1 \\
\hline$C(15 A)-C(16 A)-C(20 A)$ & 122.1 \\
\hline$C(17 A)-C(16 A)-C(20 A)$ & 108.1 \\
\hline$C(11 A)-C(17 A)-C(16 A)$ & 122.3 \\
\hline$C(11 A)-C(17 A)-C(18 A)$ & 122.1 \\
\hline$C(16 A)-C(17 A)-C(18 A)$ & 108.0 \\
\hline$C(12 A)-C(18 A)-C(19 A)$ & 122.2 \\
\hline$C(12 A)-C(18 A)-C(17 A)$ & 122.2 \\
\hline$C(19 A)-C(18 A)-C(17 A)$ & 108.0 \\
\hline$C(13 A)-C(19 A)-C(20 A)$ & 122.1 \\
\hline$C(13 A)-C(19 A)-C(18 A)$ & 122.2 \\
\hline$C(20 A)-C(19 A)-C(18 A)$ & 108.0 \\
\hline$C(14 A)-C(20 A)-C(19 A)$ & 122.2 \\
\hline$C(14 A)-C(20 A)-C(16 A)$ & 122.2 \\
\hline$C(19 A)-C(20 A)-C(16 A)$ & 107.9 \\
\hline$C(2 B)-C(1 B)-C(11 B)$ & 121.5 \\
\hline$C(2 B)-C(1 B)-P t(1 B)$ & $122.6(5)$ \\
\hline$C(11 B)-C(1 B)-P t(1 B)$ & $115.8(6)$ \\
\hline$C(1 B)-C(2 B)-C(12 B)$ & 121.6 \\
\hline$C(1 B)-C(2 B)-B r(2 B)$ & $120.8(10)$ \\
\hline$C(12 B)-C(2 B)-B r(2 B)$ & $117.5(10)$ \\
\hline$C(4 B)-C(3 B)-C(12 B)$ & 121.6 \\
\hline$C(3 B)-C(4 B)-C(13 B)$ & 121.5 \\
\hline$C(6 B)-C(5 B)-C(13 B)$ & 121.5 \\
\hline$C(6 B)-C(5 B)-B r(5 B)$ & $120.7(5)$ \\
\hline$C(13 B)-C(5 B)-B r(5 B)$ & $117.8(5)$ \\
\hline$C(5 B)-C(6 B)-C(14 B)$ & 121.5 \\
\hline$C(5 B)-C(6 B)-B r(6 B)$ & $121.1(6)$ \\
\hline$C(14 B)-C(6 B)-B r(6 B)$ & $117.3(6)$ \\
\hline$C(8 B)-C(7 B)-C(14 B)$ & 121.5 \\
\hline$C(7 B)-C(8 B)-C(15 B)$ & 121.6 \\
\hline$C(10 B)-C(9 B)-C(15 B)$ & 121.5 \\
\hline$C(9 B)-C(10 B)-C(11 B)$ & 121.6 \\
\hline$C(17 B)-C(11 B)-C(10 B)$ & 114.9 \\
\hline$C(17 B)-C(11 B)-C(1 B)$ & 114.9 \\
\hline$C(10 B)-C(11 B)-C(1 B)$ & 129.6 \\
\hline$C(18 B)-C(12 B)-C(2 B)$ & 115.0 \\
\hline$C(18 B)-C(12 B)-C(3 B)$ & 114.8 \\
\hline$C(2 B)-C(12 B)-C(3 B)$ & 129.6 \\
\hline$C(19 B)-C(13 B)-C(5 B)$ & 115.0 \\
\hline$C(19 B)-C(13 B)-C(4 B)$ & 114.9 \\
\hline$C(5 B)-C(13 B)-C(4 B)$ & 129.5 \\
\hline
\end{tabular}




\begin{tabular}{|c|c|}
\hline$C(20 B)-C(14 B)-C(6 B)$ & 115.0 \\
\hline$C(20 B)-C(14 B)-C(7 B)$ & 114.9 \\
\hline$C(6 B)-C(14 B)-C(7 B)$ & 129.6 \\
\hline$C(16 B)-C(15 B)-C(8 B)$ & 115.0 \\
\hline$C(16 B)-C(15 B)-C(9 B)$ & 114.9 \\
\hline$C(8 B)-C(15 B)-C(9 B)$ & 129.6 \\
\hline$C(15 B)-C(16 B)-C(20 B)$ & 122.2 \\
\hline$C(15 B)-C(16 B)-C(17 B)$ & 122.2 \\
\hline$C(20 B)-C(16 B)-C(17 B)$ & 108.0 \\
\hline$C(11 B)-C(17 B)-C(16 B)$ & 122.1 \\
\hline$C(11 B)-C(17 B)-C(18 B)$ & 122.2 \\
\hline$C(16 B)-C(17 B)-C(18 B)$ & 108.0 \\
\hline$C(12 B)-C(18 B)-C(19 B)$ & 122.3 \\
\hline$C(12 B)-C(18 B)-C(17 B)$ & 122.1 \\
\hline$C(19 B)-C(18 B)-C(17 B)$ & 108.0 \\
\hline$C(13 B)-C(19 B)-C(18 B)$ & 122.1 \\
\hline$C(13 B)-C(19 B)-C(20 B)$ & 122.2 \\
\hline$C(18 B)-C(19 B)-C(20 B)$ & 108.0 \\
\hline$C(14 B)-C(20 B)-C(16 B)$ & 122.2 \\
\hline$C(14 B)-C(20 B)-C(19 B)$ & 122.1 \\
\hline$C(16 B)-C(20 B)-C(19 B)$ & 108.0 \\
\hline$C(2 C)-C(1 C)-C(11 C)$ & 121.6 \\
\hline$C(2 C)-C(1 C)-P t(1 C)$ & $122.7(6)$ \\
\hline$C(11 C)-C(1 C)-P t(1 C)$ & $114.5(6)$ \\
\hline$C(1 C)-C(2 C)-C(12 C)$ & 121.6 \\
\hline$C(1 C)-C(2 C)-B r(2 C)$ & $120.2(10)$ \\
\hline$C(12 C)-C(2 C)-B r(2 C)$ & $118.1(9)$ \\
\hline$C(4 C)-C(3 C)-C(12 C)$ & 121.6 \\
\hline$C(3 C)-C(4 C)-C(13 C)$ & 121.5 \\
\hline$C(6 C)-C(5 C)-C(13 C)$ & 121.5 \\
\hline$C(6 C)-C(5 C)-B r(5 C)$ & $122.3(6)$ \\
\hline$C(13 C)-C(5 C)-B r(5 C)$ & $116.2(6)$ \\
\hline$C(5 C)-C(6 C)-C(14 C)$ & 121.5 \\
\hline$C(5 C)-C(6 C)-B r(6 C)$ & $118.7(6)$ \\
\hline$C(14 C)-C(6 C)-B r(6 C)$ & $119.7(6)$ \\
\hline$C(8 C)-C(7 C)-C(14 C)$ & 121.6 \\
\hline$C(7 C)-C(8 C)-C(15 C)$ & 121.5 \\
\hline$C(10 C)-C(9 C)-C(15 C)$ & 121.5 \\
\hline$C(9 C)-C(10 C)-C(11 C)$ & 121.6 \\
\hline$C(17 C)-C(11 C)-C(10 C)$ & 114.9 \\
\hline$C(17 C)-C(11 C)-C(1 C)$ & 114.9 \\
\hline$C(10 C)-C(11 C)-C(1 C)$ & 129.6 \\
\hline$C(18 C)-C(12 C)-C(2 C)$ & 114.8 \\
\hline$C(18 C)-C(12 C)-C(3 C)$ & 114.9 \\
\hline$C(2 C)-C(12 C)-C(3 C)$ & 129.7 \\
\hline$C(19 C)-C(13 C)-C(5 C)$ & 115.0 \\
\hline$C(19 C)-C(13 C)-C(4 C)$ & 114.9 \\
\hline$C(5 C)-C(13 C)-C(4 C)$ & 129.6 \\
\hline$C(20 C)-C(14 C)-C(6 C)$ & 115.0 \\
\hline$C(20 C)-C(14 C)-C(7 C)$ & 114.9 \\
\hline$C(6 C)-C(14 C)-C(7 C)$ & 129.5 \\
\hline$C(16 C)-C(15 C)-C(9 C)$ & 114.9 \\
\hline$C(16 C)-C(15 C)-C(8 C)$ & 114.9 \\
\hline$C(9 C)-C(15 C)-C(8 C)$ & 129.6 \\
\hline$C(15 C)-C(16 C)-C(20 C)$ & 122.2 \\
\hline$C(15 C)-C(16 C)-C(17 C)$ & 122.1 \\
\hline$C(20 C)-C(16 C)-C(17 C)$ & 108.0 \\
\hline
\end{tabular}




\begin{tabular}{|c|c|}
\hline$C(11 C)-C(17 C)-C(16 C)$ & 122.2 \\
\hline$C(11 C)-C(17 C)-C(18 C)$ & 122.2 \\
\hline$C(16 C)-C(17 C)-C(18 C)$ & 108.0 \\
\hline$C(12 C)-C(18 C)-C(19 C)$ & 122.1 \\
\hline$C(12 C)-C(18 C)-C(17 C)$ & 122.2 \\
\hline$C(19 C)-C(18 C)-C(17 C)$ & 108.1 \\
\hline$C(13 C)-C(19 C)-C(18 C)$ & 122.2 \\
\hline$C(13 C)-C(19 C)-C(20 C)$ & 122.2 \\
\hline$C(18 C)-C(19 C)-C(20 C)$ & 107.9 \\
\hline$C(14 C)-C(20 C)-C(16 C)$ & 122.2 \\
\hline$C(14 C)-C(20 C)-C(19 C)$ & 122.0 \\
\hline$C(16 C)-C(20 C)-C(19 C)$ & 108.1 \\
\hline$C(2 D)-C(1 D)-C(11 D)$ & 121.5 \\
\hline$C(2 D)-C(1 D)-P t(1 D)$ & $123.4(4)$ \\
\hline$C(11 D)-C(1 D)-P t(1 D)$ & $114.4(4)$ \\
\hline$C(1 D)-C(2 D)-C(12 D)$ & 121.6 \\
\hline$C(1 D)-C(2 D)-B r(2 D)$ & $119.5(4)$ \\
\hline $\mathrm{C}(12 \mathrm{D})-\mathrm{C}(2 \mathrm{D})-\mathrm{Br}(2 \mathrm{D})$ & $118.9(4)$ \\
\hline$C(4 D)-C(3 D)-C(12 D)$ & 121.7 \\
\hline$C(3 D)-C(4 D)-C(13 D)$ & 121.5 \\
\hline$C(6 D)-C(5 D)-C(13 D)$ & 121.6 \\
\hline$C(6 D)-C(5 D)-B r(5 D)$ & $119.8(5)$ \\
\hline$C(13 D)-C(5 D)-B r(5 D)$ & $118.5(5)$ \\
\hline$C(5 D)-C(6 D)-C(14 D)$ & 121.5 \\
\hline$C(5 D)-C(6 D)-B r(6 D)$ & $122.8(5)$ \\
\hline $\mathrm{C}(14 \mathrm{D})-\mathrm{C}(6 \mathrm{D})-\mathrm{Br}(6 \mathrm{D})$ & $115.6(5)$ \\
\hline$C(8 D)-C(7 D)-C(14 D)$ & 121.5 \\
\hline$C(7 D)-C(8 D)-C(15 D)$ & 121.6 \\
\hline$C(10 D)-C(9 D)-C(15 D)$ & 121.5 \\
\hline$C(9 D)-C(10 D)-C(11 D)$ & 121.6 \\
\hline$C(17 D)-C(11 D)-C(1 D)$ & 114.9 \\
\hline$C(17 D)-C(11 D)-C(10 D)$ & 114.9 \\
\hline$C(1 D)-C(11 D)-C(10 D)$ & 129.6 \\
\hline$C(18 D)-C(12 D)-C(3 D)$ & 114.9 \\
\hline$C(18 D)-C(12 D)-C(2 D)$ & 114.9 \\
\hline$C(3 D)-C(12 D)-C(2 D)$ & 129.7 \\
\hline$C(19 D)-C(13 D)-C(5 D)$ & 114.9 \\
\hline$C(19 D)-C(13 D)-C(4 D)$ & 114.9 \\
\hline$C(5 D)-C(13 D)-C(4 D)$ & 129.7 \\
\hline$C(20 D)-C(14 D)-C(6 D)$ & 114.9 \\
\hline$C(20 D)-C(14 D)-C(7 D)$ & 114.9 \\
\hline$C(6 D)-C(14 D)-C(7 D)$ & 129.6 \\
\hline$C(16 D)-C(15 D)-C(9 D)$ & 115.0 \\
\hline$C(16 D)-C(15 D)-C(8 D)$ & 114.9 \\
\hline$C(9 D)-C(15 D)-C(8 D)$ & 129.6 \\
\hline$C(15 D)-C(16 D)-C(20 D)$ & 122.2 \\
\hline$C(15 D)-C(16 D)-C(17 D)$ & 122.1 \\
\hline$C(20 D)-C(16 D)-C(17 D)$ & 108.0 \\
\hline$C(11 D)-C(17 D)-C(16 D)$ & 122.2 \\
\hline$C(11 D)-C(17 D)-C(18 D)$ & 122.1 \\
\hline$C(16 D)-C(17 D)-C(18 D)$ & 108.0 \\
\hline$C(12 D)-C(18 D)-C(19 D)$ & 122.2 \\
\hline$C(12 D)-C(18 D)-C(17 D)$ & 122.2 \\
\hline$C(19 D)-C(18 D)-C(17 D)$ & 108.0 \\
\hline$C(13 D)-C(19 D)-C(18 D)$ & 122.1 \\
\hline$C(13 D)-C(19 D)-C(20 D)$ & 122.1 \\
\hline$C(18 D)-C(19 D)-C(20 D)$ & 108.1 \\
\hline
\end{tabular}




\begin{tabular}{|c|c|}
\hline$C(14 D)-C(20 D)-C(16 D)$ & 122.1 \\
\hline$C(14 D)-C(20 D)-C(19 D)$ & 122.2 \\
\hline$C(16 D)-C(20 D)-C(19 D)$ & 108.0 \\
\hline$C(2 W)-C(1 W)-C(11 W)$ & 121.6 \\
\hline$C(2 W)-C(1 W)-\operatorname{Pt}(1 A)$ & $124.2(10)$ \\
\hline$C(11 W)-C(1 W)-P t(1 A)$ & $114.2(10)$ \\
\hline$C(1 W)-C(2 W)-C(12 W)$ & 121.5 \\
\hline$C(1 W)-C(2 W)-B r(2 W)$ & $122.3(12)$ \\
\hline$C(12 W)-C(2 W)-B r(2 W)$ & $116.1(12)$ \\
\hline$C(4 W)-C(3 W)-C(12 W)$ & 121.5 \\
\hline$C(3 W)-C(4 W)-C(13 W)$ & 121.5 \\
\hline$C(6 W)-C(5 W)-C(13 W)$ & 121.5 \\
\hline$C(6 W)-C(5 W)-B r(5 W)$ & $120.2(11)$ \\
\hline$C(13 W)-C(5 W)-B r(5 W)$ & $118.2(11)$ \\
\hline$C(5 W)-C(6 W)-C(14 W)$ & 121.6 \\
\hline$C(5 W)-C(6 W)-B r(6 W)$ & $119.3(12)$ \\
\hline$C(14 W)-C(6 W)-B r(6 W)$ & $118.9(12)$ \\
\hline$C(8 W)-C(7 W)-C(14 W)$ & 121.5 \\
\hline$C(7 W)-C(8 W)-C(15 W)$ & 121.6 \\
\hline$C(10 W)-C(9 W)-C(15 W)$ & 121.5 \\
\hline$C(9 W)-C(10 W)-C(11 W)$ & 121.6 \\
\hline$C(16 W)-C(11 W)-C(10 W)$ & 114.9 \\
\hline$C(16 W)-C(11 W)-C(1 W)$ & 114.8 \\
\hline$C(10 W)-C(11 W)-C(1 W)$ & 129.6 \\
\hline$C(17 W)-C(12 W)-C(2 W)$ & 114.9 \\
\hline$C(17 W)-C(12 W)-C(3 W)$ & 114.9 \\
\hline$C(2 W)-C(12 W)-C(3 W)$ & 129.6 \\
\hline$C(18 W)-C(13 W)-C(4 W)$ & 115.0 \\
\hline$C(18 W)-C(13 W)-C(5 W)$ & 114.9 \\
\hline$C(4 W)-C(13 W)-C(5 W)$ & 129.5 \\
\hline$C(19 W)-C(14 W)-C(7 W)$ & 114.9 \\
\hline$C(19 W)-C(14 W)-C(6 W)$ & 114.9 \\
\hline$C(7 W)-C(14 W)-C(6 W)$ & 129.6 \\
\hline$C(20 W)-C(15 W)-C(8 W)$ & 114.9 \\
\hline$C(20 W)-C(15 W)-C(9 W)$ & 114.9 \\
\hline$C(8 W)-C(15 W)-C(9 W)$ & 129.6 \\
\hline$C(11 W)-C(16 W)-C(20 W)$ & 122.1 \\
\hline$C(11 W)-C(16 W)-C(17 W)$ & 122.2 \\
\hline$C(20 W)-C(16 W)-C(17 W)$ & 108.0 \\
\hline$C(12 W)-C(17 W)-C(16 W)$ & 122.1 \\
\hline$C(12 W)-C(17 W)-C(18 W)$ & 122.2 \\
\hline$C(16 \mathrm{~W})-\mathrm{C}(17 \mathrm{~W})-\mathrm{C}(18 \mathrm{~W})$ & 108.0 \\
\hline$C(13 W)-C(18 W)-C(19 W)$ & 122.2 \\
\hline$C(13 W)-C(18 W)-C(17 W)$ & 122.1 \\
\hline$C(19 W)-C(18 W)-C(17 W)$ & 108.0 \\
\hline$C(14 W)-C(19 W)-C(20 W)$ & 122.2 \\
\hline$C(14 W)-C(19 W)-C(18 W)$ & 122.1 \\
\hline $\mathrm{C}(20 \mathrm{~W})-\mathrm{C}(19 \mathrm{~W})-\mathrm{C}(18 \mathrm{~W})$ & 108.0 \\
\hline$C(15 W)-C(20 W)-C(19 W)$ & 122.1 \\
\hline$C(15 W)-C(20 W)-C(16 W)$ & 122.2 \\
\hline$C(19 W)-C(20 W)-C(16 W)$ & 108.0 \\
\hline$C(2 Y)-C(1 Y)-C(11 Y)$ & 121.4 \\
\hline$C(2 Y)-C(1 Y)-B r(1 Y)$ & $121(2)$ \\
\hline$C(11 Y)-C(1 Y)-B r(1 Y)$ & $117(2)$ \\
\hline$C(1 Y)-C(2 Y)-C(12 Y)$ & 121.5 \\
\hline$C(1 Y)-C(2 Y)-P t(1 C)$ & $121.4(11)$ \\
\hline$C(12 Y)-C(2 Y)-P t(1 C)$ & $116.8(11)$ \\
\hline
\end{tabular}




\begin{tabular}{|c|c|}
\hline$C(4 Y)-C(3 Y)-C(12 Y)$ & 121.4 \\
\hline$C(3 Y)-C(4 Y)-C(13 Y)$ & 121.6 \\
\hline$C(6 Y)-C(5 Y)-C(13 Y)$ & 121.4 \\
\hline$C(6 Y)-C(5 Y)-B r(5 Y)$ & $115.2(13)$ \\
\hline$C(13 Y)-C(5 Y)-B r(5 Y)$ & $122.2(13)$ \\
\hline$C(5 Y)-C(6 Y)-C(14 Y)$ & 121.7 \\
\hline $\mathrm{C}(5 \mathrm{Y})-\mathrm{C}(6 \mathrm{Y})-\mathrm{Br}(6 \mathrm{Y})$ & $124.7(13)$ \\
\hline$C(14 Y)-C(6 Y)-\operatorname{Br}(6 Y)$ & $113.4(13)$ \\
\hline$C(8 Y)-C(7 Y)-C(14 Y)$ & 121.6 \\
\hline$C(7 Y)-C(8 Y)-C(15 Y)$ & 121.5 \\
\hline$C(10 Y)-C(9 Y)-C(15 Y)$ & 121.4 \\
\hline$C(9 Y)-C(10 Y)-C(11 Y)$ & 121.6 \\
\hline$C(16 Y)-C(11 Y)-C(10 Y)$ & 115.0 \\
\hline C (16Y)-C (11Y)-C (1Y) & 115.0 \\
\hline$C(10 Y)-C(11 Y)-C(1 Y)$ & 129.5 \\
\hline$C(17 Y)-C(12 Y)-C(2 Y)$ & 115.0 \\
\hline$C(17 Y)-C(12 Y)-C(3 Y)$ & 115.0 \\
\hline $\mathrm{C}(2 \mathrm{Y})-\mathrm{C}(12 \mathrm{Y})-\mathrm{C}(3 \mathrm{Y})$ & 129.5 \\
\hline$C(18 Y)-C(13 Y)-C(4 Y)$ & 114.9 \\
\hline$C(18 Y)-C(13 Y)-C(5 Y)$ & 114.9 \\
\hline$C(4 Y)-C(13 Y)-C(5 Y)$ & 129.6 \\
\hline $\mathrm{C}(19 \mathrm{Y})-\mathrm{C}(14 \mathrm{Y})-\mathrm{C}(6 \mathrm{Y})$ & 114.9 \\
\hline$C(19 Y)-C(14 Y)-C(7 Y)$ & 114.9 \\
\hline$C(6 Y)-C(14 Y)-C(7 Y)$ & 129.6 \\
\hline$C(20 Y)-C(15 Y)-C(8 Y)$ & 115.0 \\
\hline$C(20 Y)-C(15 Y)-C(9 Y)$ & 114.9 \\
\hline$C(8 Y)-C(15 Y)-C(9 Y)$ & 129.5 \\
\hline$C(11 Y)-C(16 Y)-C(20 Y)$ & 122.1 \\
\hline$C(11 Y)-C(16 Y)-C(17 Y)$ & 122.2 \\
\hline$C(20 Y)-C(16 Y)-C(17 Y)$ & 108.0 \\
\hline$C(12 Y)-C(17 Y)-C(16 Y)$ & 122.1 \\
\hline $\mathrm{C}(12 \mathrm{Y})-\mathrm{C}(17 \mathrm{Y})-\mathrm{C}(18 \mathrm{Y})$ & 122.2 \\
\hline$C(16 Y)-C(17 Y)-C(18 Y)$ & 108.0 \\
\hline$C(13 Y)-C(18 Y)-C(17 Y)$ & 122.1 \\
\hline C (13Y) -C (18Y) -C (19Y) & 122.2 \\
\hline $\mathrm{C}(17 \mathrm{Y})-\mathrm{C}(18 \mathrm{Y})-\mathrm{C}(19 \mathrm{Y})$ & 108.0 \\
\hline$C(14 Y)-C(19 Y)-C(20 Y)$ & 122.3 \\
\hline$C(14 Y)-C(19 Y)-C(18 Y)$ & 122.1 \\
\hline$C(20 Y)-C(19 Y)-C(18 Y)$ & 107.9 \\
\hline$C(15 Y)-C(20 Y)-C(19 Y)$ & 122.1 \\
\hline$C(15 Y)-C(20 Y)-C(16 Y)$ & 122.2 \\
\hline$C(19 Y)-C(20 Y)-C(16 Y)$ & 108.0 \\
\hline$C(2 X)-C(1 X)-C(11 X)$ & 121.6 \\
\hline$C(2 X)-C(1 X)-B r(1 X)$ & $124(3)$ \\
\hline$C(11 X)-C(1 X)-B r(1 X)$ & $114(3)$ \\
\hline$C(1 X)-C(2 X)-C(12 X)$ & 121.5 \\
\hline$C(1 X)-C(2 X)-P t(1 B)$ & $118.6(17)$ \\
\hline$C(12 X)-C(2 X)-P t(1 B)$ & $119.8(17)$ \\
\hline$C(4 X)-C(3 X)-C(12 X)$ & 121.6 \\
\hline$C(3 X)-C(4 X)-C(13 X)$ & 121.5 \\
\hline$C(6 X)-C(5 X)-C(13 X)$ & 121.5 \\
\hline$C(6 X)-C(5 X)-B r(5 X)$ & $118.0(17)$ \\
\hline$C(13 X)-C(5 X)-B r(5 X)$ & $118.5(17)$ \\
\hline$C(5 X)-C(6 X)-C(14 X)$ & 121.6 \\
\hline$C(5 X)-C(6 X)-B r(6 X)$ & $122.9(16)$ \\
\hline
\end{tabular}




\begin{tabular}{|c|c|}
\hline$C(14 X)-C(6 X)-B r(6 X)$ & $114.7(16)$ \\
\hline$C(8 X)-C(7 X)-C(14 X)$ & 121.5 \\
\hline$C(7 X)-C(8 X)-C(15 X)$ & 121.6 \\
\hline$C(10 X)-C(9 X)-C(15 X)$ & 121.6 \\
\hline$C(9 X)-C(10 X)-C(11 X)$ & 121.5 \\
\hline$C(16 x)-C(11 X)-C(10 X)$ & 114.9 \\
\hline$C(16 X)-C(11 X)-C(1 X)$ & 114.9 \\
\hline$C(10 X)-C(11 X)-C(1 X)$ & $129.59(5)$ \\
\hline$C(17 X)-C(12 X)-C(3 X)$ & 114.9 \\
\hline$C(17 X)-C(12 X)-C(2 X)$ & 114.9 \\
\hline$C(3 X)-C(12 X)-C(2 X)$ & 129.6 \\
\hline$C(18 X)-C(13 X)-C(5 X)$ & 115.0 \\
\hline$C(18 X)-C(13 X)-C(4 X)$ & 114.9 \\
\hline$C(5 X)-C(13 X)-C(4 X)$ & 129.6 \\
\hline$C(19 X)-C(14 X)-C(7 X)$ & 115.0 \\
\hline$C(19 X)-C(14 X)-C(6 X)$ & 114.8 \\
\hline$C(7 X)-C(14 X)-C(6 x)$ & 129.6 \\
\hline$C(20 X)-C(15 X)-C(8 X)$ & 114.9 \\
\hline$C(20 X)-C(15 X)-C(9 X)$ & 114.9 \\
\hline$C(8 X)-C(15 X)-C(9 X)$ & 129.6 \\
\hline$C(11 X)-C(16 X)-C(17 X)$ & 122.1 \\
\hline$C(11 X)-C(16 X)-C(20 X)$ & 122.2 \\
\hline$C(17 X)-C(16 X)-C(20 X)$ & 108.0 \\
\hline$C(12 X)-C(17 X)-C(18 X)$ & 122.1 \\
\hline$C(12 X)-C(17 X)-C(16 X)$ & 122.2 \\
\hline$C(18 X)-C(17 X)-C(16 X)$ & 107.9 \\
\hline$C(13 X)-C(18 X)-C(19 X)$ & 122.1 \\
\hline$C(13 X)-C(18 X)-C(17 X)$ & 122.1 \\
\hline$C(19 X)-C(18 X)-C(17 X)$ & 108.1 \\
\hline$C(14 X)-C(19 X)-C(18 X)$ & 122.3 \\
\hline$C(14 X)-C(19 X)-C(20 X)$ & 122.1 \\
\hline$C(18 X)-C(19 X)-C(20 X)$ & 108.0 \\
\hline$C(15 X)-C(20 X)-C(16 X)$ & 122.2 \\
\hline$C(15 X)-C(20 X)-C(19 X)$ & 122.2 \\
\hline$C(16 X)-C(20 X)-C(19 X)$ & 107.9 \\
\hline$C(2 Z)-C(1 Z)-C(11 Z)$ & 121.6 \\
\hline$C(1 Z)-C(2 Z)-C(12 Z)$ & 121.6 \\
\hline$C(1 Z)-C(2 Z)-P t(1 D)$ & $113(2)$ \\
\hline$C(12 Z)-C(2 Z)-P t(1 D)$ & $124(2)$ \\
\hline$C(4 Z)-C(3 Z)-C(12 Z)$ & 121.5 \\
\hline$C(3 Z)-C(4 Z)-C(13 Z)$ & 121.6 \\
\hline$C(6 Z)-C(5 Z)-C(13 Z)$ & 121.6 \\
\hline$C(6 Z)-C(5 Z)-B r(5 Z)$ & $125(2)$ \\
\hline$C(13 Z)-C(5 z)-B r(5 z)$ & $113(2)$ \\
\hline$C(5 Z)-C(6 Z)-C(14 Z)$ & 121.5 \\
\hline$C(5 Z)-C(6 Z)-B r(6 Z)$ & $119(2)$ \\
\hline$C(14 Z)-C(6 Z)-B r(6 Z)$ & $119(2)$ \\
\hline$C(8 Z)-C(7 Z)-C(14 Z)$ & 121.5 \\
\hline$C(7 Z)-C(8 Z)-C(15 Z)$ & 121.6 \\
\hline$C(10 Z)-C(9 Z)-C(15 Z)$ & 121.6 \\
\hline C (9Z) -C (10Z) -C (11Z) & 121.6 \\
\hline$C(16 Z)-C(11 Z)-C(10 Z)$ & 114.8 \\
\hline$C(16 Z)-C(11 Z)-C(1 Z)$ & 114.9 \\
\hline$C(10 Z)-C(11 Z)-C(1 Z)$ & 129.7 \\
\hline$C(17 Z)-C(12 Z)-C(3 Z)$ & 114.9 \\
\hline$C(17 Z)-C(12 Z)-C(2 Z)$ & 114.8 \\
\hline$C(3 Z)-C(12 Z)-C(2 Z)$ & 129.7 \\
\hline
\end{tabular}




\begin{tabular}{|c|c|}
\hline$C(18 Z)-C(13 Z)-C(4 Z)$ & 114.9 \\
\hline$C(18 Z)-C(13 Z)-C(5 Z)$ & 114.9 \\
\hline$C(4 Z)-C(13 Z)-C(5 Z)$ & 129.6 \\
\hline$C(19 Z)-C(14 Z)-C(6 z)$ & 115.0 \\
\hline$C(19 Z)-C(14 z)-C(7 Z)$ & 115.0 \\
\hline$C(6 Z)-C(14 Z)-C(7 Z)$ & 129.5 \\
\hline$C(20 Z)-C(15 Z)-C(9 Z)$ & 115.0 \\
\hline$C(20 z)-C(15 z)-C(8 Z)$ & 114.8 \\
\hline C (9Z) -C (15Z)-C (8Z) & 129.6 \\
\hline$C(11 Z)-C(16 Z)-C(20 Z)$ & 122.2 \\
\hline$C(11 Z)-C(16 Z)-C(17 Z)$ & 122.1 \\
\hline$C(20 Z)-C(16 Z)-C(17 Z)$ & 108.0 \\
\hline$C(12 Z)-C(17 Z)-C(18 Z)$ & 122.2 \\
\hline$C(12 Z)-C(17 Z)-C(16 Z)$ & 122.2 \\
\hline$C(18 z)-C(17 z)-C(16 z)$ & 108.0 \\
\hline$C(13 z)-C(18 z)-C(17 z)$ & 122.1 \\
\hline$C(13 z)-C(18 Z)-C(19 Z)$ & 122.2 \\
\hline$C(17 Z)-C(18 Z)-C(19 Z)$ & 108.0 \\
\hline$C(14 z)-C(19 z)-C(20 z)$ & 122.1 \\
\hline$C(14 Z)-C(19 Z)-C(18 Z)$ & 122.2 \\
\hline$C(20 Z)-C(19 z)-C(18 Z)$ & 108.0 \\
\hline$C(15 z)-C(20 z)-C(19 z)$ & 122.2 \\
\hline$C(15 z)-C(20 z)-C(16 z)$ & 122.1 \\
\hline$C(19 z)-C(20 z)-C(16 z)$ & 108.0 \\
\hline
\end{tabular}

Symmetry transformations used to generate equivalent atoms: \#1 $-\mathrm{x}+1,-\mathrm{y},-\mathrm{z}+1 \quad \# 2 \mathrm{x}-1 / 2,-\mathrm{y}+1 / 2, \mathrm{z}-1 / 2$

\#3 $x+1 / 2,-y+1 / 2, z+1 / 2$ 
Table 11. Anisotropic displacement parameters ( $\left.A^{\wedge} 2 \times 10^{\wedge} 3\right)$ for trans $-3 /$ trans -4 .

The anisotropic displacement factor exponent takes the form:

$-2 \mathrm{pi}^{\wedge} 2\left[\mathrm{~h}^{\wedge} 2 \mathrm{a}^{\star \wedge} 2 \mathrm{U11}+\ldots+2 \mathrm{~h} \mathrm{k} \mathrm{a}^{\star} \mathrm{b}\right.$ * U12]

\begin{tabular}{|c|c|c|c|c|c|c|}
\hline & $\mathrm{U} 11$ & U22 & U33 & U23 & U13 & U12 \\
\hline Pt (1A) & $20(1)$ & $43(1)$ & $39(1)$ & $0(1)$ & $4(1)$ & $0(1)$ \\
\hline Pt (1B) & $38(1)$ & $50(1)$ & $42(1)$ & $7(1)$ & $8(1)$ & $10(1)$ \\
\hline Pt (1C) & $26(1)$ & $46(1)$ & $57(1)$ & $2(1)$ & 1 (1) & $-5(1)$ \\
\hline Pt (1D) & $35(1)$ & $48(1)$ & $56(1)$ & $-9(1)$ & $-2(1)$ & $-6(1)$ \\
\hline$P(1 A)$ & $20(4)$ & $56(5)$ & $48(4)$ & $9(3)$ & $3(3)$ & $-1(3)$ \\
\hline$P(2 A)$ & $26(4)$ & $46(4)$ & $56(4)$ & $0(3)$ & $0(3)$ & $-8(3)$ \\
\hline $\mathrm{P}(1 \mathrm{~B})$ & $35(4)$ & $47(4)$ & $59(4)$ & $17(3)$ & $10(3)$ & $4(4)$ \\
\hline$P(2 B)$ & $79(6)$ & $65(6)$ & $64(5)$ & $16(4)$ & $28(4)$ & $38(5)$ \\
\hline$P(1 C)$ & $27(4)$ & $53(5)$ & $73(5)$ & $13(4)$ & $-5(4)$ & $-4(4)$ \\
\hline$P(2 C)$ & $50(5)$ & $59(6)$ & $101(6)$ & $7(5)$ & $-17(5)$ & $-13(4)$ \\
\hline$P(1 D)$ & $38(5)$ & $78(6)$ & $75(5)$ & $-8(4)$ & $6(4)$ & $-16(4)$ \\
\hline$P(2 D)$ & $55(5)$ & $49(5)$ & $88(6)$ & $-19(4)$ & $-4(4)$ & $-15(4)$ \\
\hline$C(21 A)$ & $20(14)$ & $55(17)$ & $45(15)$ & $-7(12)$ & $-7(11)$ & $-5(12)$ \\
\hline$C(23 A)$ & $90(30)$ & $80(20)$ & $70(20)$ & $18(18)$ & $-7(19)$ & $40(20)$ \\
\hline$C(24 A)$ & $100(30)$ & $140(30)$ & $43(18)$ & $-37(19)$ & $9(17)$ & $-30(20)$ \\
\hline$C(25 A)$ & $20(15)$ & $40(17)$ & $140(30)$ & $-3(17)$ & $-6(17)$ & $-16(13)$ \\
\hline$C(26 A)$ & $100(30)$ & $100(30)$ & $80(20)$ & $27(19)$ & $50(20)$ & $40(20)$ \\
\hline$C(27 A)$ & $32(16)$ & $80(20)$ & $80(20)$ & $16(17)$ & $36(15)$ & $-1(15)$ \\
\hline$C(28 A)$ & $90(30)$ & $100(30)$ & $70(20)$ & 32 (19) & $-29(19)$ & $-10(20)$ \\
\hline$C(29 A)$ & $20(15)$ & $63(19)$ & $90(20)$ & 12 (15) & $-12(14)$ & $-26(14)$ \\
\hline$C(30 A)$ & $43(17)$ & $57(18)$ & $90(20)$ & 12 (15) & $-53(15)$ & $-25(15)$ \\
\hline$C(31 A)$ & $90(30)$ & $38(18)$ & $100(20)$ & $-35(16)$ & $-10(20)$ & $8(17)$ \\
\hline$C(32 A)$ & $50(20)$ & $250(50)$ & $50(20)$ & $-30(20)$ & $39(17)$ & $0(20)$ \\
\hline$C(21 B)$ & 27 (17) & $50(20)$ & $150(30)$ & $14(19)$ & $9(18)$ & $-19(15)$ \\
\hline$C(22 B)$ & $110(30)$ & $120(30)$ & $90(30)$ & $30(20)$ & $-50(20)$ & $-40(30)$ \\
\hline$C(23 B)$ & $13(13)$ & $30(14)$ & $67(16)$ & $10(12)$ & $-9(12)$ & $2(11)$ \\
\hline$C(24 B)$ & $24(15)$ & $70(20)$ & 61 (17) & $23(14)$ & 2 (13) & $11(14)$ \\
\hline$C(25 B)$ & $100(30)$ & $80(20)$ & $60(20)$ & $23(16)$ & $60(18)$ & $50(20)$ \\
\hline$C(26 B)$ & $240(50)$ & $100(30)$ & $50(20)$ & $-3(19)$ & $10(20)$ & $30(30)$ \\
\hline$C(28 B)$ & $110(40)$ & $100(30)$ & $470(80)$ & $120(40)$ & $170(50)$ & $60(30)$ \\
\hline$C(29 B)$ & $160(40)$ & $50(20)$ & $120(30)$ & $-10(20)$ & $50(30)$ & $20(20)$ \\
\hline$C(30 B)$ & $80(30)$ & $80(30)$ & $160(40)$ & $-30(30)$ & $80(30)$ & $-10(20)$ \\
\hline$C(31 B)$ & $100(30)$ & $70(20)$ & $140(30)$ & $-10(20)$ & $80(20)$ & $10(20)$ \\
\hline$C(32 B)$ & $140(40)$ & $110(30)$ & $130(30)$ & $40(20)$ & $80(30)$ & $20(30)$ \\
\hline$C(21 C)$ & 12 (17) & $100(30)$ & $380(60)$ & $-100(40)$ & $30(30)$ & $-2(19)$ \\
\hline$C(23 C)$ & $80(30)$ & $300(60)$ & $290(60)$ & $200(60)$ & $-130(40)$ & $-120(40)$ \\
\hline$C(24 C)$ & $110(30)$ & $270(60)$ & $60(30)$ & $50(30)$ & $0(20)$ & $-30(30)$ \\
\hline$C(26 C)$ & $210(50)$ & $120(30)$ & $70(20)$ & $20(20)$ & $60(30)$ & $60(30)$ \\
\hline$C(27 C)$ & $100(30)$ & $50(20)$ & $220(40)$ & $-40(30)$ & $-130(30)$ & $0(20)$ \\
\hline$C(28 C)$ & $100(30)$ & $150(40)$ & $140(40)$ & $-80(30)$ & $-40(30)$ & $10(30)$ \\
\hline$C(29 C)$ & 42 (18) & $60(20)$ & $100(20)$ & $-6(16)$ & $-15(17)$ & $-21(16)$ \\
\hline$C(30 C)$ & 31 (17) & $100(30)$ & $100(20)$ & $29(19)$ & $-1(16)$ & $-20(17)$ \\
\hline$C(31 C)$ & $50(30)$ & $180(50)$ & $230(50)$ & $80(40)$ & $-30(30)$ & $-70(30)$ \\
\hline$C(32 C)$ & $150(40)$ & $210(50)$ & $80(30)$ & $-10(30)$ & $40(30)$ & $-40(40)$ \\
\hline$C(21 D)$ & $620(130)$ & $350(90)$ & $140(50)$ & $-10(50)$ & $0(60)$ & $-430(100)$ \\
\hline$C(22 D)$ & $220(60)$ & $190(50)$ & $350(80)$ & $-120(50)$ & $240(60)$ & $-100(40)$ \\
\hline
\end{tabular}




\begin{tabular}{|c|c|c|c|c|c|c|}
\hline$C(23 D)$ & $28(18)$ & $120(30)$ & $180(40)$ & $-90(30)$ & $40(20)$ & $-20(20)$ \\
\hline$C(24 D)$ & $260(60)$ & $120(40)$ & $190(50)$ & $-70(30)$ & $-110(50)$ & $100(40)$ \\
\hline$C(25 D)$ & $60(20)$ & $22(16)$ & $240(40)$ & $0(20)$ & $30(20)$ & $-27(16)$ \\
\hline$C(27 D)$ & $80(20)$ & $70(20)$ & $120(30)$ & $-50(20)$ & $-10(20)$ & $-23(19)$ \\
\hline$C(28 D)$ & $80(30)$ & $100(30)$ & $190(40)$ & $10(30)$ & $-30(30)$ & $-20(20)$ \\
\hline$C(29 D)$ & $90(30)$ & $40(20)$ & $170(40)$ & $0(20)$ & $20(30)$ & $-41(19)$ \\
\hline$C(30 D)$ & $100(30)$ & $80(30)$ & $130(30)$ & $40(20)$ & $10(20)$ & $0(20)$ \\
\hline$C(31 D)$ & $70(20)$ & $33(17)$ & $110(20)$ & $-6(15)$ & $11(18)$ & $0(16)$ \\
\hline$C(32 D)$ & $70(20)$ & $140(30)$ & $150(30)$ & $40(30)$ & $30(20)$ & $-50(20)$ \\
\hline $\operatorname{Br}(1 \mathrm{~A})$ & $49(6)$ & $62(6)$ & $39(4)$ & $0(4)$ & $11(3)$ & $8(4)$ \\
\hline $\operatorname{Br}(2 A)$ & $26(4)$ & $47(4)$ & $32(5)$ & $10(4)$ & $6(4)$ & $4(3)$ \\
\hline $\operatorname{Br}(5 A)$ & $53(3)$ & $107(5)$ & $57(3)$ & $-28(3)$ & $2(2)$ & $23(3)$ \\
\hline $\operatorname{Br}(6 A)$ & $58(4)$ & $69(5)$ & $112(5)$ & $-39(4)$ & $-7(4)$ & $26(4)$ \\
\hline $\operatorname{Br}(1 \mathrm{~B})$ & $64(6)$ & $44(5)$ & $57(5)$ & $9(4)$ & $13(4)$ & $-4(3)$ \\
\hline $\operatorname{Br}(2 B)$ & $42(8)$ & $65(6)$ & $46(4)$ & $-1(4)$ & $3(4)$ & $-1(6)$ \\
\hline $\operatorname{Br}(5 B)$ & $51(2)$ & $71(3)$ & $81(3)$ & $17(2)$ & $15(2)$ & $-9(2)$ \\
\hline $\operatorname{Br}(6 \mathrm{~B})$ & $48(2)$ & $53(2)$ & $91(3)$ & $-7(2)$ & $14(2)$ & $-12(2)$ \\
\hline $\operatorname{Br}(1 \mathrm{C})$ & $39(4)$ & $64(5)$ & $79(7)$ & $5(5)$ & $-4(4)$ & $-1(3)$ \\
\hline $\operatorname{Br}(2 \mathrm{C})$ & $43(8)$ & $86(5)$ & $64(5)$ & $-6(4)$ & $-6(4)$ & $11(6)$ \\
\hline $\operatorname{Br}(5 C)$ & $85(4)$ & $74(3)$ & $87(3)$ & $21(3)$ & $-8(3)$ & $28(3)$ \\
\hline $\operatorname{Br}(6 C)$ & $68(3)$ & $56(3)$ & $107(4)$ & $-7(3)$ & $3(3)$ & $27(3)$ \\
\hline $\operatorname{Br}(1 D)$ & $65(2)$ & $70(2)$ & $126(3)$ & $-39(2)$ & $0(2)$ & $8(2)$ \\
\hline $\operatorname{Br}(5 D)$ & $65(3)$ & $72(3)$ & $92(3)$ & $-21(2)$ & $39(2)$ & $7(2)$ \\
\hline $\operatorname{Br}(6 D)$ & $42(2)$ & $51(2)$ & $106(3)$ & $8(2)$ & $15(2)$ & $16(2)$ \\
\hline $\operatorname{Br}(1 \mathrm{~W})$ & $49(10)$ & $57(7)$ & $76(17)$ & $16(13)$ & $0(12)$ & $9(7)$ \\
\hline $\operatorname{Br}(2 \mathrm{~W})$ & $54(9)$ & $97(11)$ & $45(6)$ & $-11(7)$ & $-6(6)$ & $23(7)$ \\
\hline $\operatorname{Br}(5 W)$ & $75(6)$ & $63(6)$ & $136(8)$ & $34(5)$ & $-28(5)$ & $14(5)$ \\
\hline $\operatorname{Br}(6 \mathrm{~W})$ & $81(7)$ & $50(7)$ & $159(11)$ & $-39(6)$ & $-36(7)$ & $49(6)$ \\
\hline $\operatorname{Br}(1 X)$ & $24(16)$ & $61(16)$ & $24(10)$ & $0(10)$ & $-2(10)$ & $-5(15)$ \\
\hline $\operatorname{Br}(2 X)$ & $34(11)$ & $53(18)$ & $66(15)$ & $4(14)$ & $-4(10)$ & $16(14)$ \\
\hline $\operatorname{Br}(5 X)$ & $72(10)$ & $97(11)$ & $30(7)$ & $-21(7)$ & $-5(6)$ & $-12(9)$ \\
\hline $\operatorname{Br}(6 X)$ & $45(9)$ & $72(10)$ & $96(12)$ & $-28(8)$ & $10(8)$ & $0(8)$ \\
\hline $\operatorname{Br}(1 Y)$ & $33(14)$ & $76(12)$ & $74(9)$ & $12(7)$ & $18(8)$ & $12(11)$ \\
\hline $\operatorname{Br}(5 \mathrm{Y})$ & $115(11)$ & $105(10)$ & $73(8)$ & $-22(6)$ & $52(7)$ & $-22(8)$ \\
\hline $\operatorname{Br}(6 \mathrm{Y})$ & $79(9)$ & $52(8)$ & $184(16)$ & $-44(9)$ & $66(10)$ & $14(7)$ \\
\hline $\operatorname{Br}(5 z)$ & $62(15)$ & $85(17)$ & $53(13)$ & $40(11)$ & $7(10)$ & $18(12)$ \\
\hline $\operatorname{Br}(6 \mathrm{Z})$ & $51(14)$ & $34(12)$ & $95(19)$ & $17(11)$ & $28(13)$ & $12(10)$ \\
\hline
\end{tabular}




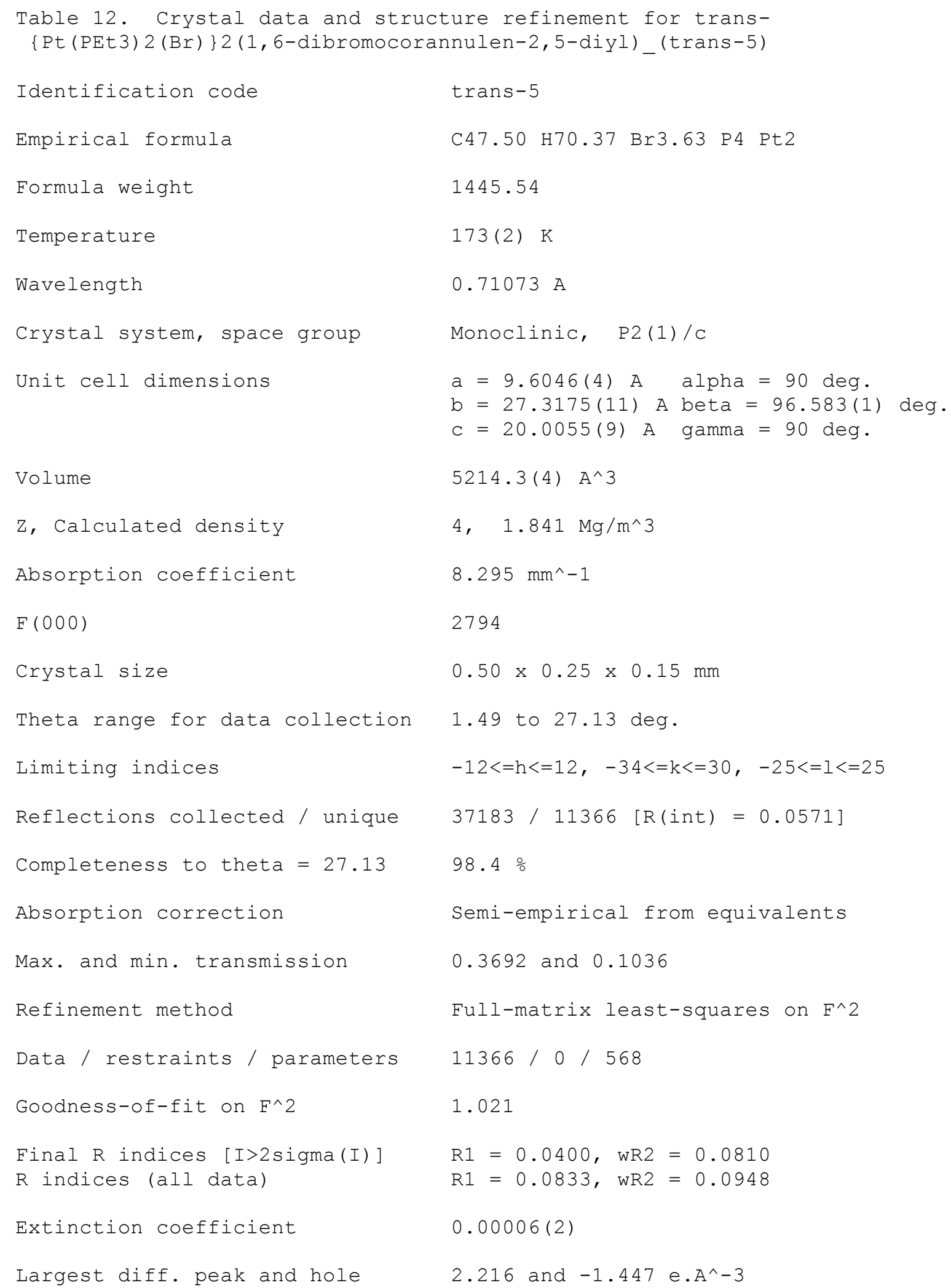


Table 13. Atomic coordinates $\left(x 10^{\wedge} 4\right)$ and equivalent isotropic displacement parameters $\left(A^{\wedge} 2 \times 10^{\wedge} 3\right)$ for trans-\{Pt(PEt3) $\left.2(\mathrm{Br})\right\} 2(1,6-$ dibromocorannulen-2,5-diyl) (trans-5)

$\mathrm{U}(e q)$ is defined as one third of the trace of the orthogonalized Uij tensor.

\begin{tabular}{|c|c|c|c|c|}
\hline & $x$ & $\mathrm{Y}$ & $\mathrm{z}$ & $\mathrm{U}(\mathrm{eq})$ \\
\hline Pt (1) & $1625(1)$ & $4987(1)$ & $8122(1)$ & $28(1)$ \\
\hline Pt (2) & $835(1)$ & $7026(1)$ & $5107(1)$ & $25(1)$ \\
\hline $\operatorname{Br}(1)$ & $4048(2)$ & $5437(1)$ & $9306(1)$ & 75 (1) \\
\hline $\operatorname{Br}(2)$ & $-72(1)$ & $4317(1)$ & $8332(1)$ & $61(1)$ \\
\hline $\operatorname{Br}(5)$ & $-1144(1)$ & 7075 (1) & $4167(1)$ & $39(1)$ \\
\hline $\operatorname{Br}(6)$ & $3146(1)$ & $7959(1)$ & $5661(1)$ & $53(1)$ \\
\hline P ( 1) & 3197 (2) & $4388(1)$ & 7908 (1) & 37 (1) \\
\hline$P(2)$ & $20(3)$ & $5556(1)$ & 8395 (1) & $50(1)$ \\
\hline$P(3)$ & 2305 (3) & $6774(1)$ & $4340(1)$ & $51(1)$ \\
\hline P ( 4) & $-604(2)$ & $7308(1)$ & $5879(1)$ & $33(1)$ \\
\hline C (1) & $3978(8)$ & $5702(3)$ & $8427(4)$ & 31 (2) \\
\hline C (2) & $2980(7)$ & $5515(2)$ & $7929(3)$ & $23(2)$ \\
\hline C (3) & $1768(7)$ & $5729(2)$ & $6730(3)$ & $23(2)$ \\
\hline C (4) & $1613(7)$ & $6085(2)$ & $6226(3)$ & $24(2)$ \\
\hline C ( 5$)$ & $2431(7)$ & $6948(2)$ & $5849(3)$ & $25(2)$ \\
\hline$C(6)$ & $3367(7)$ & $7321(2)$ & $6067(3)$ & $26(2)$ \\
\hline C ( 7) & $5135(8)$ & $7654(3)$ & $7056(4)$ & $37(2)$ \\
\hline C ( 8$)$ & $5824(8)$ & 7547 (3) & $7693(4)$ & $41(2)$ \\
\hline$C(9)$ & $6101(8)$ & $6887(3)$ & $8644(4)$ & $42(2)$ \\
\hline C (10) & $5626(8)$ & $6436(3)$ & $8825(4)$ & $41(2)$ \\
\hline C (11) & $4925(7)$ & $6101(2)$ & $8339(4)$ & $30(2)$ \\
\hline C (12) & $2925(7)$ & $5731(2)$ & $7258(3)$ & $23(2)$ \\
\hline C (13) & $2618(7)$ & $6476(2)$ & $6199(3)$ & $20(1)$ \\
\hline C (14) & $4457(7)$ & $7288(3)$ & $6627(3)$ & $27(2)$ \\
\hline C (15) & $5885(7)$ & $7058(3)$ & 7952 (4) & $33(2)$ \\
\hline$C(16)$ & $5394(7)$ & $6707(3)$ & $7493(4)$ & $28(2)$ \\
\hline C (17) & $4942(7)$ & $6236(2)$ & $7676(3)$ & $27(2)$ \\
\hline C (18) & $3953(7)$ & $6062(2)$ & 7147 (3) & $22(2)$ \\
\hline C (19) & $3809(7)$ & $6419(2)$ & $6632(3)$ & $24(2)$ \\
\hline$C(20)$ & $4718(7)$ & $6812(2)$ & $6851(3)$ & $23(2)$ \\
\hline C (21) & $3495(12)$ & 3941 (3) & $8576(5)$ & $74(3)$ \\
\hline C (22) & $4146(14)$ & $4136(5)$ & $9214(5)$ & $108(5)$ \\
\hline C (23) & $2572(9)$ & $4015(3)$ & $7175(5)$ & $55(3)$ \\
\hline$C(24)$ & $2291(11)$ & $4300(4)$ & $6527(5)$ & $68(3)$ \\
\hline$C(25)$ & $4928(8)$ & $4586(3)$ & $7749(4)$ & $42(2)$ \\
\hline$C(26)$ & $6007(9)$ & $4184(3)$ & $7672(5)$ & $64(3)$ \\
\hline C (27) & $-1840(20)$ & $5427(11)$ & $8004(13)$ & $54(6)$ \\
\hline$C(28)$ & $-1850(30)$ & $5431(13)$ & 7246 (19) & $99(12)$ \\
\hline C (29) & $-153(19)$ & $5610(7)$ & $9266(7)$ & $62(5)$ \\
\hline$C(30)$ & $1160(20)$ & $5764(8)$ & $9733(7)$ & $122(9)$ \\
\hline C (31) & $438(14)$ & $6215(4)$ & $8135(7)$ & $38(3)$ \\
\hline C (32) & $-616(13)$ & $6579(4)$ & $8278(6)$ & $98(4)$ \\
\hline C (33) & 3931 (16) & $6429(7)$ & $4725(7)$ & $27(4)$ \\
\hline C (34) & $4930(20)$ & $6259(8)$ & $4244(10)$ & $43(5)$ \\
\hline$C(35)$ & $3300(20)$ & $7340(8)$ & $4002(10)$ & $64(7)$ \\
\hline
\end{tabular}




\begin{tabular}{|c|c|c|c|c|}
\hline$C(36)$ & $2570(16)$ & $7689(5)$ & $3744(7)$ & $110(5)$ \\
\hline$C(37)$ & $1750(17)$ & $6447(6)$ & $3652(8)$ & $38(5)$ \\
\hline $\mathrm{C}(38)$ & $1350(20)$ & $5922(8)$ & $3846(13)$ & $63(7)$ \\
\hline C (39) & $250(9)$ & $7558(4)$ & $6654(4)$ & $59(3)$ \\
\hline$C(40)$ & $-694(10)$ & $7796(4)$ & $7123(4)$ & $67(3)$ \\
\hline$C(41)$ & $-1666(11)$ & $6816(4)$ & $6229(6)$ & $75(3)$ \\
\hline$C(42)$ & $-2472(11)$ & $6530(4)$ & $5759(6)$ & $91(4)$ \\
\hline$C(43)$ & $-1873(11)$ & $7758(4)$ & $5565(5)$ & $74(4)$ \\
\hline C ( 44$)$ & $-1163(12)$ & $8202(4)$ & $5305(5)$ & $77(3)$ \\
\hline$C(45)$ & $4220(20)$ & $5167(5)$ & $5464(7)$ & $98(5)$ \\
\hline$C(46)$ & $5580(20)$ & $5269(5)$ & $5521(8)$ & $102(5)$ \\
\hline C ( 47) & $6440(20)$ & $5121(6)$ & $5075(11)$ & $124(6)$ \\
\hline$C(48)$ & $8240(30)$ & $5272(14)$ & $5240(20)$ & $188(19)$ \\
\hline$C\left(27^{\prime}\right)$ & $-1550(70)$ & $5510(30)$ & $8090(40)$ & $70(30)$ \\
\hline$C\left(28^{\prime}\right)$ & $-2110(60)$ & $5410(20)$ & $7300(30)$ & $43(12)$ \\
\hline$C\left(29^{\prime}\right)$ & $-270(40)$ & $5348(16)$ & $9370(20)$ & $67(12)$ \\
\hline$C\left(30^{\prime}\right)$ & $-1630(30)$ & $5443(15)$ & $9617(17)$ & $84(14)$ \\
\hline$C\left(31^{\prime}\right)$ & $140(40)$ & $6122(14)$ & $8420(20)$ & $71(13)$ \\
\hline$C\left(33^{\prime}\right)$ & $4019(18)$ & 6647 ( 8) & $4633(9)$ & $42(5)$ \\
\hline$C\left(34^{\prime}\right)$ & $4980(30)$ & $6431(9)$ & 4145 (12) & $64(7)$ \\
\hline$C\left(35^{\prime}\right)$ & $2230(20)$ & $7144(9)$ & $3614(9)$ & $72(8)$ \\
\hline C (37') & $1490(20)$ & $6172(8)$ & 3868 (11) & $45(6)$ \\
\hline$C\left(38^{\prime}\right)$ & $1280(30)$ & $5769(8)$ & $4367(14)$ & $109(12)$ \\
\hline
\end{tabular}


Table 14. Distances [A] and angles [deg] for trans\{Pt (PEt3) 2 (Br) 2 (1,6-dibromocorannulen-2,5-diyl)_(trans-5)

\begin{tabular}{|c|c|}
\hline Pt (1) $-\mathrm{C}(2)$ & $2.011(7)$ \\
\hline Pt (1) $-\mathrm{P}(1)$ & $2.299(2)$ \\
\hline Pt (1) -P (2) & $2.300(2)$ \\
\hline Pt (1) $-\operatorname{Br}(2)$ & $2.5179(9)$ \\
\hline Pt (2) $-\mathrm{C}(5)$ & $2.019(6)$ \\
\hline Pt (2) $-P(3)$ & $2.306(2)$ \\
\hline Pt (2) $-\mathrm{P}(4)$ & $2.319(2)$ \\
\hline $\operatorname{Pt}(2)-\operatorname{Br}(5)$ & $2.5203(7)$ \\
\hline $\mathrm{Br}(1)-\mathrm{C}(1)$ & $1.896(7)$ \\
\hline $\mathrm{Br}(6)-\mathrm{C}(6)$ & $1.925(6)$ \\
\hline$P(1)-C(21)$ & $1.808(9)$ \\
\hline$P(1)-C(25)$ & $1.811(8)$ \\
\hline$P(1)-C(23)$ & $1.829(9)$ \\
\hline$P(2)-C\left(31^{\prime}\right)$ & $1.55(4)$ \\
\hline$P(2)-C\left(27^{\prime}\right)$ & $1.56(6)$ \\
\hline P (2) -C (29) & $1.775(14)$ \\
\hline $\mathrm{P}(2)-\mathrm{C}(27)$ & $1.89(2)$ \\
\hline $\mathrm{P}(2)-\mathrm{C}(31)$ & $1.930(14)$ \\
\hline$P(2)-C\left(29^{\prime}\right)$ & $2.08(4)$ \\
\hline P (3) -C (37) & $1.676(15)$ \\
\hline$P(3)-C\left(33^{\prime}\right)$ & $1.719(17)$ \\
\hline$P(3)-C\left(35^{\prime}\right)$ & $1.762(18)$ \\
\hline$P(3)-C(33)$ & $1.911(15)$ \\
\hline$P(3)-C\left(37^{\prime}\right)$ & $2.01(2)$ \\
\hline$P(3)-C(35)$ & $1.98(2)$ \\
\hline$P(4)-C(43)$ & $1.793(9)$ \\
\hline P (4) -C (39) & $1.804(8)$ \\
\hline P (4) -C ( 41$)$ & $1.872(10)$ \\
\hline$C(1)-C(2)$ & $1.397(9)$ \\
\hline$C(1)-C(11)$ & $1.444(10)$ \\
\hline$C(2)-C(12)$ & $1.461(9)$ \\
\hline$C(3)-C(4)$ & $1.396(9)$ \\
\hline$C(3)-C(12)$ & $1.443(9)$ \\
\hline$C(4)-C(13)$ & $1.444(9)$ \\
\hline$C(5)-C(6)$ & $1.397(9)$ \\
\hline$C(5)-C(13)$ & $1.468(9)$ \\
\hline$C(6)-C(14)$ & $1.446(9)$ \\
\hline$C(7)-C(8)$ & $1.398(10)$ \\
\hline$C(7)-C(14)$ & $1.425(10)$ \\
\hline$C(8)-C(15)$ & $1.433(10)$ \\
\hline$C(9)-C(10)$ & $1.378(10)$ \\
\hline$C(9)-C(15)$ & $1.452(10)$ \\
\hline$C(10)-C(11)$ & $1.443(10)$ \\
\hline $\mathrm{C}(11)-\mathrm{C}(17)$ & $1.378(9)$ \\
\hline$C(12)-C(18)$ & $1.375(9)$ \\
\hline$C(13)-C(19)$ & $1.362(9)$ \\
\hline$C(14)-C(20)$ & $1.389(9)$ \\
\hline$C(15)-C(16)$ & $1.373(10)$ \\
\hline$C(16)-C(20)$ & $1.399(9)$ \\
\hline$C(16)-C(17)$ & $1.419(9)$ \\
\hline $\mathrm{C}(17)-\mathrm{C}(18)$ & $1.421(9)$ \\
\hline$C(18)-C(19)$ & $1.416(9)$ \\
\hline
\end{tabular}




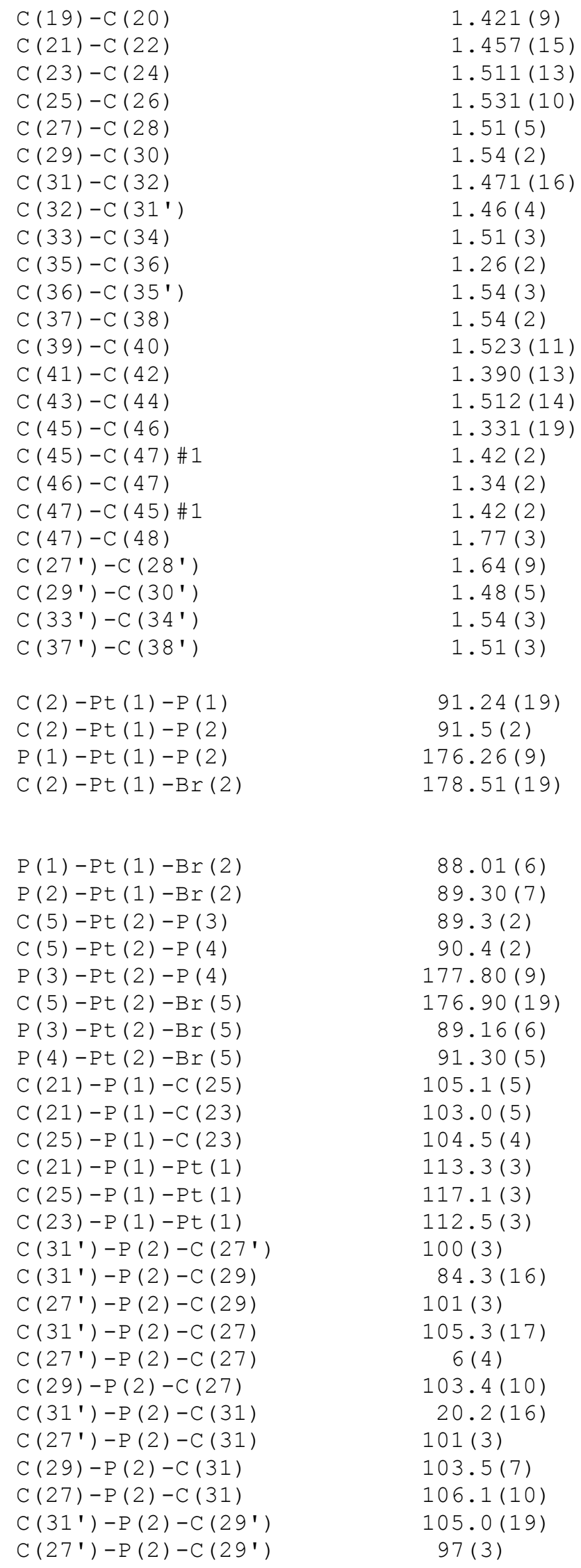




\begin{tabular}{|c|c|}
\hline$C(29)-P(2)-C\left(29^{\prime}\right)$ & $20.7(11)$ \\
\hline $\mathrm{C}(27)-\mathrm{P}(2)-\mathrm{C}\left(29^{\prime}\right)$ & $96.7(14)$ \\
\hline$C(31)-P(2)-C\left(29^{\prime}\right)$ & $124.2(12)$ \\
\hline $\mathrm{C}\left(31^{\prime}\right)-\mathrm{P}(2)-\mathrm{Pt}(1)$ & $129.2(16)$ \\
\hline $\mathrm{C}\left(27^{\prime}\right)-\mathrm{P}(2)-\mathrm{Pt}(1)$ & $119(3)$ \\
\hline $\mathrm{C}(29)-\mathrm{P}(2)-\mathrm{Pt}(1)$ & $115.5(6)$ \\
\hline$C(27)-P(2)-P t(1)$ & $113.6(8)$ \\
\hline $\mathrm{C}(31)-\mathrm{P}(2)-\mathrm{Pt}(1)$ & $113.6(4)$ \\
\hline$C\left(29^{\prime}\right)-P(2)-P t(1)$ & $101.7(11)$ \\
\hline $\mathrm{C}(37)-\mathrm{P}(3)-\mathrm{C}\left(33^{\prime}\right)$ & $111.9(8)$ \\
\hline $\mathrm{C}(37)-\mathrm{P}(3)-\mathrm{C}\left(35^{\prime}\right)$ & $69.6(9)$ \\
\hline$C\left(33^{\prime}\right)-P(3)-C\left(35^{\prime}\right)$ & $110.3(9)$ \\
\hline$C(37)-P(3)-C(33)$ & $103.6(7)$ \\
\hline$C\left(33^{\prime}\right)-P(3)-C(33)$ & $19.1(7)$ \\
\hline$C\left(35^{\prime}\right)-P(3)-C(33)$ & $124.8(8)$ \\
\hline $\mathrm{C}(37)-\mathrm{P}(3)-\mathrm{C}\left(37^{\prime}\right)$ & $26.8(7)$ \\
\hline$C\left(33^{\prime}\right)-P(3)-C\left(37^{\prime}\right)$ & $107.6(9)$ \\
\hline$C\left(35^{\prime}\right)-P(3)-C\left(37^{\prime}\right)$ & $96.0(10)$ \\
\hline$C(33)-P(3)-C\left(37^{\prime}\right)$ & $92.4(8)$ \\
\hline $\mathrm{C}(37)-\mathrm{P}(3)-\mathrm{C}(35)$ & $104.9(9)$ \\
\hline$C\left(33^{\prime}\right)-P(3)-C(35)$ & $78.1(9)$ \\
\hline$C\left(35^{\prime}\right)-P(3)-C(35)$ & $41.2(9)$ \\
\hline$C(33)-P(3)-C(35)$ & $96.8(8)$ \\
\hline$C\left(37^{\prime}\right)-P(3)-C(35)$ & $131.1(9)$ \\
\hline $\mathrm{C}(37)-\mathrm{P}(3)-\mathrm{Pt}(2)$ & $123.0(6)$ \\
\hline $\mathrm{C}\left(33^{\prime}\right)-\mathrm{P}(3)-\mathrm{Pt}(2)$ & $117.9(6)$ \\
\hline $\mathrm{C}\left(35^{\prime}\right)-\mathrm{P}(3)-\mathrm{Pt}(2)$ & $113.9(7)$ \\
\hline $\mathrm{C}(33)-\mathrm{P}(3)-\mathrm{Pt}(2)$ & $114.4(5)$ \\
\hline $\mathrm{C}\left(37^{\prime}\right)-\mathrm{P}(3)-\operatorname{Pt}(2)$ & $108.7(6)$ \\
\hline$C(35)-P(3)-P t(2)$ & $110.6(6)$ \\
\hline$C(43)-P(4)-C(39)$ & $105.4(4)$ \\
\hline$C(43)-P(4)-C(41)$ & $104.2(5)$ \\
\hline$C(39)-P(4)-C(41)$ & $99.3(5)$ \\
\hline$C(43)-P(4)-P t(2)$ & $115.4(3)$ \\
\hline $\mathrm{C}(39)-\mathrm{P}(4)-\mathrm{Pt}(2)$ & $116.8(3)$ \\
\hline$C(41)-P(4)-P t(2)$ & $113.7(3)$ \\
\hline$C(2)-C(1)-C(11)$ & $125.4(6)$ \\
\hline$C(2)-C(1)-B r(1)$ & $118.1(5)$ \\
\hline$C(11)-C(1)-B r(1)$ & $116.5(5)$ \\
\hline$C(1)-C(2)-C(12)$ & $117.2(6)$ \\
\hline$C(1)-C(2)-P t(1)$ & $122.1(5)$ \\
\hline$C(12)-C(2)-\operatorname{Pt}(1)$ & $120.6(5)$ \\
\hline$C(4)-C(3)-C(12)$ & $122.5(6)$ \\
\hline$C(3)-C(4)-C(13)$ & $121.6(6)$ \\
\hline$C(6)-C(5)-C(13)$ & $117.0(6)$ \\
\hline$C(6)-C(5)-\operatorname{Pt}(2)$ & $124.0(5)$ \\
\hline$C(13)-C(5)-\operatorname{Pt}(2)$ & $119.0(5)$ \\
\hline$C(5)-C(6)-C(14)$ & $125.4(6)$ \\
\hline$C(5)-C(6)-B r(6)$ & $119.6(5)$ \\
\hline$C(14)-C(6)-\operatorname{Br}(6)$ & $114.8(5)$ \\
\hline$C(8)-C(7)-C(14)$ & $122.6(7)$ \\
\hline$C(7)-C(8)-C(15)$ & $121.3(7)$ \\
\hline$C(10)-C(9)-C(15)$ & $121.5(7)$ \\
\hline$C(9)-C(10)-C(11)$ & $122.1(7)$ \\
\hline$C(17)-C(11)-C(10)$ & $115.1(7)$ \\
\hline $\mathrm{C}(17)-\mathrm{C}(11)-\mathrm{C}(1)$ & $113.4(6)$ \\
\hline$C(10)-C(11)-C(1)$ & $130.3(7)$ \\
\hline
\end{tabular}




\begin{tabular}{|c|c|}
\hline$C(18)-C(12)-C(3)$ & $113.0(6)$ \\
\hline$C(18)-C(12)-C(2)$ & $117.5(6)$ \\
\hline$C(3)-C(12)-C(2)$ & $128.0(6)$ \\
\hline$C(19)-C(13)-C(4)$ & $114.1(6)$ \\
\hline$C(19)-C(13)-C(5)$ & $116.8(6)$ \\
\hline$C(4)-C(13)-C(5)$ & $128.4(6)$ \\
\hline$C(20)-C(14)-C(7)$ & $114.2(6)$ \\
\hline$C(20)-C(14)-C(6)$ & $113.4(6)$ \\
\hline$C(7)-C(14)-C(6)$ & $131.5(7)$ \\
\hline$C(16)-C(15)-C(8)$ & $114.3(7)$ \\
\hline$C(16)-C(15)-C(9)$ & $114.5(7)$ \\
\hline$C(8)-C(15)-C(9)$ & $129.8(7)$ \\
\hline$C(15)-C(16)-C(20)$ & $124.0(7)$ \\
\hline$C(15)-C(16)-C(17)$ & $123.4(7)$ \\
\hline$C(20)-C(16)-C(17)$ & $107.4(6)$ \\
\hline$C(11)-C(17)-C(16)$ & $121.9(6)$ \\
\hline$C(11)-C(17)-C(18)$ & $123.1(7)$ \\
\hline$C(16)-C(17)-C(18)$ & $108.1(6)$ \\
\hline$C(12)-C(18)-C(19)$ & $124.0(6)$ \\
\hline $\mathrm{C}(12)-\mathrm{C}(18)-\mathrm{C}(17)$ & $122.2(6)$ \\
\hline$C(19)-C(18)-C(17)$ & $108.2(6)$ \\
\hline $\mathrm{C}(13)-\mathrm{C}(19)-\mathrm{C}(18)$ & $123.1(6)$ \\
\hline$C(13)-C(19)-C(20)$ & $123.6(6)$ \\
\hline$C(18)-C(19)-C(20)$ & $106.9(6)$ \\
\hline$C(14)-C(20)-C(16)$ & $122.4(6)$ \\
\hline$C(14)-C(20)-C(19)$ & $121.7(6)$ \\
\hline$C(16)-C(20)-C(19)$ & $109.5(6)$ \\
\hline $\mathrm{C}(22)-\mathrm{C}(21)-\mathrm{P}(1)$ & $114.6(8)$ \\
\hline$C(24)-C(23)-P(1)$ & $114.3(6)$ \\
\hline$C(26)-C(25)-P(1)$ & $116.7(6)$ \\
\hline$C(28)-C(27)-P(2)$ & $108.1(18)$ \\
\hline$C(30)-C(29)-P(2)$ & $117.1(12)$ \\
\hline$C(32)-C(31)-P(2)$ & $114.1(10)$ \\
\hline$C\left(31^{\prime}\right)-C(32)-C(31)$ & $28.3(16)$ \\
\hline$C(34)-C(33)-P(3)$ & $116.5(12)$ \\
\hline$C(36)-C(35)-P(3)$ & $117.6(16)$ \\
\hline$C(35)-C(36)-C\left(35^{\prime}\right)$ & $55.7(13)$ \\
\hline$C(38)-C(37)-P(3)$ & $110.8(14)$ \\
\hline$C(40)-C(39)-P(4)$ & $116.5(6)$ \\
\hline$C(42)-C(41)-P(4)$ & $115.8(9)$ \\
\hline$C(44)-C(43)-P(4)$ & $110.7(8)$ \\
\hline$C(46)-C(45)-C(47) \# 1$ & $121.7(14)$ \\
\hline$C(45)-C(46)-C(47)$ & $123.6(16)$ \\
\hline$C(46)-C(47)-C(45) \# 1$ & $114.8(16)$ \\
\hline$C(46)-C(47)-C(48)$ & $118(2)$ \\
\hline C (45) \#1-C (47)-C (48) & $127(3)$ \\
\hline$P(2)-C\left(27^{\prime}\right)-C\left(28^{\prime}\right)$ & $126(5)$ \\
\hline$C\left(30^{\prime}\right)-C\left(29^{\prime}\right)-P(2)$ & $119(3)$ \\
\hline$C(32)-C\left(31^{\prime}\right)-P(2)$ & $144(3)$ \\
\hline $\mathrm{C}\left(34^{\prime}\right)-\mathrm{C}\left(33^{\prime}\right)-\mathrm{P}(3)$ & $118.9(14)$ \\
\hline$C(36)-C\left(35^{\prime}\right)-P(3)$ & $115.2(13)$ \\
\hline $\mathrm{C}\left(38^{\prime}\right)-\mathrm{C}\left(37^{\prime}\right)-\mathrm{P}(3)$ & $111.1(13)$ \\
\hline
\end{tabular}

Symmetry transformations used to generate equivalent atoms: \# $1-x+1,-y+1,-z+1$ 
Table 15. Anisotropic displacement parameters $\left(A^{\wedge} 2 \times 10^{\wedge} 3\right)$ for trans$\{$ Pt (PEt3) 2 (Br) 2 2 (1,6-dibromocorannulen-2,5-diyl)_(trans-5)

The anisotropic displacement factor exponent takes the form:

$-2 \mathrm{pi}^{\wedge} 2\left[\mathrm{~h}^{\wedge} 2 \mathrm{a}^{\star \wedge} 2 \mathrm{U} 11+\ldots+2 \mathrm{~h} \mathrm{k} \mathrm{a}^{\star} \mathrm{b}\right.$ * U12 ]

\begin{tabular}{|c|c|c|c|c|c|c|}
\hline & $\mathrm{U} 11$ & $\mathrm{U} 22$ & U33 & U23 & U13 & $\mathrm{U} 12$ \\
\hline Pt (1) & $33(1)$ & $22(1)$ & $30(1)$ & $6(1)$ & $7(1)$ & $4(1)$ \\
\hline Pt (2) & $25(1)$ & $26(1)$ & $23(1)$ & $4(1)$ & $1(1)$ & $4(1)$ \\
\hline $\operatorname{Br}(1)$ & $133(2)$ & $54(1)$ & $28(1)$ & $15(1)$ & $-30(1)$ & $-42(1)$ \\
\hline $\operatorname{Br}(2)$ & $48(1)$ & $52(1)$ & $87(1)$ & $26(1)$ & $20(1)$ & $-8(1)$ \\
\hline $\operatorname{Br}(5)$ & $37(1)$ & $45(1)$ & $31(1)$ & $-2(1)$ & $-7(1)$ & $10(1)$ \\
\hline $\operatorname{Br}(6)$ & $58(1)$ & $37(1)$ & $60(1)$ & $21(1)$ & $-4(1)$ & $-8(1)$ \\
\hline P (1) & $40(1)$ & $21(1)$ & $52(1)$ & $3(1)$ & $13(1)$ & $6(1)$ \\
\hline$P(2)$ & $44(1)$ & $56(2)$ & $48(1)$ & $-11(1)$ & $1(1)$ & $22(1)$ \\
\hline$P(3)$ & $59(2)$ & $65(2)$ & $30(1)$ & $7(1)$ & $14(1)$ & $25(1)$ \\
\hline P (4) & $28(1)$ & $34(1)$ & $36(1)$ & $-4(1)$ & $2(1)$ & $5(1)$ \\
\hline$C(1)$ & $37(4)$ & $24(4)$ & $32(4)$ & $10(3)$ & $-1(4)$ & $4(3)$ \\
\hline$C(2)$ & $35(4)$ & $13(3)$ & $22(3)$ & $2(3)$ & $2(3)$ & $4(3)$ \\
\hline C (3) & $28(4)$ & $15(3)$ & $26(4)$ & $-2(3)$ & $1(3)$ & $0(3)$ \\
\hline$C(4)$ & $23(4)$ & $25(4)$ & $25(4)$ & $-4(3)$ & $3(3)$ & $-3(3)$ \\
\hline$C(5)$ & $22(4)$ & $26(4)$ & $27(3)$ & $7(3)$ & $4(3)$ & $5(3)$ \\
\hline$C(6)$ & $24(4)$ & $20(4)$ & $33(4)$ & $10(3)$ & $1(3)$ & $-4(3)$ \\
\hline$C(7)$ & $39(5)$ & $26(4)$ & $45(5)$ & $12(4)$ & $1(4)$ & $-12(4)$ \\
\hline$C(8)$ & $27(4)$ & $37(5)$ & $59(6)$ & $-6(4)$ & $3(4)$ & $-13(4)$ \\
\hline$C(9)$ & $39(5)$ & $42(5)$ & $42(5)$ & $-4(4)$ & $-9(4)$ & $-13(4)$ \\
\hline$C(10)$ & $41(5)$ & $46(5)$ & $30(4)$ & $6(4)$ & $-15(4)$ & $-7(4)$ \\
\hline C (11) & $26(4)$ & $26(4)$ & $35(4)$ & $8(3)$ & $-3(3)$ & $6(3)$ \\
\hline C (12) & $31(4)$ & $12(3)$ & $25(4)$ & $1(3)$ & $4(3)$ & $8(3)$ \\
\hline C (13) & $22(4)$ & $27(4)$ & $13(3)$ & $3(3)$ & $5(3)$ & $3(3)$ \\
\hline C (14) & $20(4)$ & $32(4)$ & $30(4)$ & $5(3)$ & $5(3)$ & $-5(3)$ \\
\hline$C(15)$ & $19(4)$ & $31(4)$ & $47(5)$ & $5(4)$ & $-1(3)$ & $-10(3)$ \\
\hline$C(16)$ & $11(3)$ & $34(4)$ & $37(4)$ & $2(3)$ & $-1(3)$ & $-2(3)$ \\
\hline C (17) & $26(4)$ & $25(4)$ & $29(4)$ & $1(3)$ & $2(3)$ & $4(3)$ \\
\hline$C(18)$ & $27(4)$ & $20(4)$ & $19(3)$ & $4(3)$ & $2(3)$ & $9(3)$ \\
\hline$C(19)$ & $30(4)$ & $20(4)$ & $22(3)$ & $0(3)$ & $7(3)$ & $0(3)$ \\
\hline C (20) & $16(3)$ & $28(4)$ & $25(3)$ & $1(3)$ & $7(3)$ & $3(3)$ \\
\hline$C(21)$ & $92(8)$ & $52(6)$ & $86(8)$ & $30(6)$ & $42(7)$ & $37(6)$ \\
\hline C (22) & $139(12)$ & $130(12)$ & $51(7)$ & $35(7)$ & $-5(8)$ & $59(10)$ \\
\hline C (23) & $51(6)$ & $31(5)$ & $85(7)$ & $-18(5)$ & $13(5)$ & $-9(4)$ \\
\hline$C(24)$ & $64(7)$ & $75(7)$ & $64(7)$ & $-33(6)$ & $6(6)$ & $-3(6)$ \\
\hline C (25) & $35(5)$ & $41(5)$ & $53(5)$ & $-13(4)$ & $9(4)$ & $5(4)$ \\
\hline$C(26)$ & $38(5)$ & $67(7)$ & $84(7)$ & $-28(6)$ & $2(5)$ & $14(5)$ \\
\hline C (27) & $25(9)$ & 68 (13) & $67(14)$ & $-16(11)$ & $-6(9)$ & $11(10)$ \\
\hline$C(28)$ & $22(12)$ & $150(20)$ & $110(20)$ & $30(15)$ & $-24(13)$ & $8(13)$ \\
\hline C (29) & $99(13)$ & $52(11)$ & $41(8)$ & $3(8)$ & $29(9)$ & $9(9)$ \\
\hline$C(30)$ & $180(20)$ & $170(20)$ & $22(8)$ & $-16(10)$ & $25(11)$ & $-41(17)$ \\
\hline$C(31)$ & $41(7)$ & $32(7)$ & $46(8)$ & $-1(6)$ & $24(7)$ & $11(5)$ \\
\hline C (32) & $100(10)$ & $84(9)$ & $108(10)$ & $27(8)$ & $-2(8)$ & $22(8)$ \\
\hline$C(35)$ & $63(15)$ & $86(16)$ & $48(12)$ & $16(11)$ & $33(11)$ & $35(12)$ \\
\hline$C(36)$ & $133(13)$ & $90(10)$ & $113(12)$ & $22(9)$ & $34(10)$ & $-22(10)$ \\
\hline C (38) & $63(14)$ & $35(13)$ & $86(18)$ & $13(13)$ & $-7(12)$ & $2(11)$ \\
\hline C (39) & $49(6)$ & $101(8)$ & $28(4)$ & $-6(5)$ & $2(4)$ & $17(5)$ \\
\hline
\end{tabular}




\begin{tabular}{|c|c|c|c|c|c|c|}
\hline$C(40)$ & $72(7)$ & $92(8)$ & $32(5)$ & $-18(5)$ & $-8(5)$ & $30(6)$ \\
\hline$C(41)$ & $60(7)$ & $80(8)$ & $90(8)$ & $-10(7)$ & $29(6)$ & $-7(6)$ \\
\hline$C(42)$ & $56(7)$ & 101 (9) & $123(11)$ & $-20(8)$ & $34(7)$ & $-45(7)$ \\
\hline$C(43)$ & $82(8)$ & $93(8)$ & $42(5)$ & $-18(5)$ & $-19(5)$ & $53(7)$ \\
\hline$C(44)$ & $130(11)$ & $45(6)$ & $56(6)$ & $12(5)$ & $11(7)$ & $26(6)$ \\
\hline$C(45)$ & $186(17)$ & $48(7)$ & $70(9)$ & $10(7)$ & $62(11)$ & $50(9)$ \\
\hline$C(46)$ & $155(16)$ & $70(9)$ & $81(11)$ & $5(8)$ & $7(11)$ & $-19(11)$ \\
\hline C (47) & $161(17)$ & $84(12)$ & $128(15)$ & $33(11)$ & $22(14)$ & $17(11)$ \\
\hline C (48) & $49(17)$ & $240(40)$ & $280(50)$ & $90(40)$ & $20(20)$ & $-10(20)$ \\
\hline$C\left(30^{\prime}\right)$ & $40(20)$ & $150(40)$ & $60(20)$ & $-10(20)$ & $16(17)$ & $40(20)$ \\
\hline$C\left(35^{\prime}\right)$ & $40(11)$ & $150(20)$ & $25(9)$ & $22(12)$ & $9(9)$ & $21(13)$ \\
\hline$C\left(38^{\prime}\right)$ & $170(30)$ & 51 (15) & $90(20)$ & $-16(14)$ & $-60(20)$ & $10(16)$ \\
\hline
\end{tabular}

